Review

\title{
The Cusp-Core Problem in Gas-Poor Dwarf Spheroidal Galaxies
}

\author{
Pierre Boldrini ${ }^{1,2}$
}

1 Université de Lorraine, CNRS, Inria, LORIA, F-54000 Nancy, France; boldrini@iap.fr

2 Institut d'Astrophysique de Paris, Sorbonne Université, CNRS, UMR 7095, 98 Bis Bd Arago, F-75014 Paris, France

\begin{abstract}
This review deals with the inconsistency of inner dark matter density profiles in dwarf galaxies, known as the cusp-core problem. In particular, we aim to focus on gas-poor dwarf galaxies. One of the most promising solutions to this cold dark matter small-scale issue is the stellar feedback, but it seems to be only designed for gas-rich dwarfs. However, in the regime of classical dwarfs, this core mechanism becomes negligible. Therefore, it is required to find solutions without invoking these baryonic processes as dark matter cores tend to persist even for these dwarfs, which are rather darkmatter-dominated. Here, we have presented two categories of solutions. One consists of creating dark matter cores from cusps within cold dark matter by altering the dark matter potential via perturbers. The second category gathers solutions that depict the natural emergence of dark matter cores in alternative theories. Given the wide variety of solutions, it becomes necessary to identify which mechanism dominates in the central region of galaxies by finding observational signatures left by them in order to highlight the true nature of dark matter.
\end{abstract}

Keywords: dark matter; dwarf spheroidal galaxies; alternative theories; stellar feedback

\section{Introduction}

Citation: Boldrini, P. The Cusp-Core Problem in Gas-Poor Dwarf Spheroidal Galaxies. Galaxies 2022, 10 , 5. https://doi.org/10.3390/ galaxies10010005

Academic Editors: Behnam

Javanmardi and Yanbin Yang

Received: 28 October 2021

Accepted: 22 December 2021

Published: 30 December 2021

Publisher's Note: MDPI stays neutral with regard to jurisdictional claims in published maps and institutional affiliations.

Copyright: (C) 2021 by the authors. Licensee MDPI, Basel, Switzerland. This article is an open access article distributed under the terms and conditions of the Creative Commons Attribution (CC BY) license (https:// creativecommons.org/licenses/by/ $4.0 /)$.

\subsection{Lambda Cold Dark Matter Paradigm and Its Dark Matter Cusps}

The nature of dark matter (DM) is currently one of the most fundamental and elusive mysteries in physics. One way to constrain the nature of the DM is to understand how DM is distributed in galaxies. The DM is arranged, particularly in the centre of galaxies, according to the properties that we attribute to it. In the prevailing cosmological theory, Lambda Cold Dark Matter $(\Lambda \mathrm{CDM})$, a collisionless and non-relativistic non-luminous matter, spans our entire Universe [1]. However, DM could be more complex and hotter than simple CDM. Indeed, DM could be an ultra-light scalar field or self-interacting or have several components. Nevertheless, the CDM paradigm can provide a quantitative description of the Universe at present and is extremely successful at explaining the Universe on large scales [2,3], as well as many important aspects of galaxy formation $[4,5]$.

CDM cosmological simulations including only DM particles predict that DM halos should have density profiles that behave as $r^{-1}$ at small radii. Halo mergers gradually drive the halo density profiles towards a central density cusp with a sharp decline towards their outskirts [6-8]. These early simulations of structure formation found a universal cuspy density profile in halos ranging from dwarf galaxies to galaxy clusters [9]. This density profile, which is almost independent of halo mass, cosmological parameters and the power spectrum of initial fluctuations, appeared to be well-described by the following form $[9,10]$ (hereafter NFW):

$$
\rho_{\mathrm{NFW}}(r)=\frac{\rho_{0}}{\left(\frac{r}{r_{\mathrm{s}}}\right)\left(1+\frac{r}{r_{\mathrm{s}}}\right)^{2}},
$$

where $r$ is the distance from the centre of the DM halo, and $\rho_{0}$ and $r_{\mathrm{S}}$ represent the central density and scale radius, respectively. The NFW profile is a double power law that 
transitions from $r^{-1}$ at small radii to $r^{-3}$ at large radii (see Equation (1)). The scale radius marks the transition between the two slopes in the NFW profile. As the NFW profile appears to be the generic consequence of halo mergers and becomes more resilient, this might explain why this density profile is universally observed in most cosmological simulations. Nevertheless, later studies show that the DM density profile does not seem to be universal. Indeed, DM density profiles rise steeply at small radii closer to $\rho(r) \propto r^{-\alpha}$ with $\alpha=0.8-1.4$ [11-13] and they are well-fitted by an Einasto profile [14], particularly for MW-like halos [15]. As the theory of the formation of our Universe dominated by the DM is established, the $\Lambda \mathrm{CDM}$ model can now be confronted with observations.

\subsection{The Historical Cusp-Core Problem}

In order to find good observational probes of the DM distribution, it is essential that the dynamics of selected galaxies are dominated by DM, i.e., with $M_{*} / M_{\mathrm{DM}}<10^{-2}$. This is the reason that the mass regime, which has been studied most extensively, is at the dwarf galaxy scale. "Dwarfs" usually refer to galaxies with $M_{*}<10^{9} \mathrm{M}_{\odot}$. Around forty dwarf galaxies have been discovered in the Local Group, which encompasses our MW galaxy and the Andromeda galaxy [16]. The dwarf population of the MW exhibits various different morphological types. Dwarfs can be divided into roughly two groups: those that lack gas and have no ongoing star formation, corresponding to dwarf spheroidals (dSphs), and those with gas and ongoing star formation are called dwarf irregulars (dIrrs) [17].

Early measurements of the HI rotation curves of gas-rich dwarf galaxies highlighted, for the first time, a large discrepancy between the observed rotation velocities and those predicted by $\Lambda C D M$ simulations, especially in the inner parts [18-20]. The DM density profile of dIrrs can be inferred by measuring the rotation of either the gas with $\mathrm{HI}$ or using the stellar $\mathrm{H} \alpha$ emission line. The rotation curve is derived from the observed line-of-sight velocity at any position in the galaxy velocity field. After data analysis, the central DM distributions in these DM-dominated galaxies were found to be inconsistent with the $1 / r$ behaviour of cuspy profiles and indicate the presence of a constant-density core. The latter implies $\rho(r) \propto r^{-\alpha}$ with $\alpha=0$ in the inner regions. This discrepancy between observations and DM-only simulations led to the original small-scale problem, which has now become known as the cusp-core problem $[18,19]$.

However, it was argued that systematic effects could be responsible for the core signature in the rotation curve [21-23]. The absence of a comprehensive and satisfactory resolution has also led to a wide range of different conclusions concerning the DM inner profile [24]. As a consequence, the NFW form cannot be ruled out [25]. Recent surveys of nearby dwarf galaxies, THINGS and LITTLE THINGS, have offered ultra-high-resolution rotation curve data [26,27]. Indeed, high-resolution velocity fields were used to derive stronger constraints on the DM distributions in galaxies [28-30]. A core profile represented by the pseudo-isothermal model is preferred over the NFW profile to explain the observational data (see Figure 1). The mass distribution of the pseudo-isothermal sphere is given by:

$$
\rho(r)=\frac{\rho_{0}}{1+\left(r / R_{\mathrm{c}}\right)^{2}}
$$

where $\rho_{0}$ and $R_{\mathrm{c}}$ are the central density and the core radius of the DM halo, respectively. By reaching the necessary resolution to alleviate some systematic effects, the logarithmic inner slope $\alpha$ of their DM halo densities was found to be approximately $\alpha=-0.32 \pm 0.24[31,32]$. Thus, these recent measurements of galaxy rotation curves in dIrrs reinforce the historical disagreement with the $\Lambda \mathrm{CDM}$ prediction at small scales (see Figure 1). 


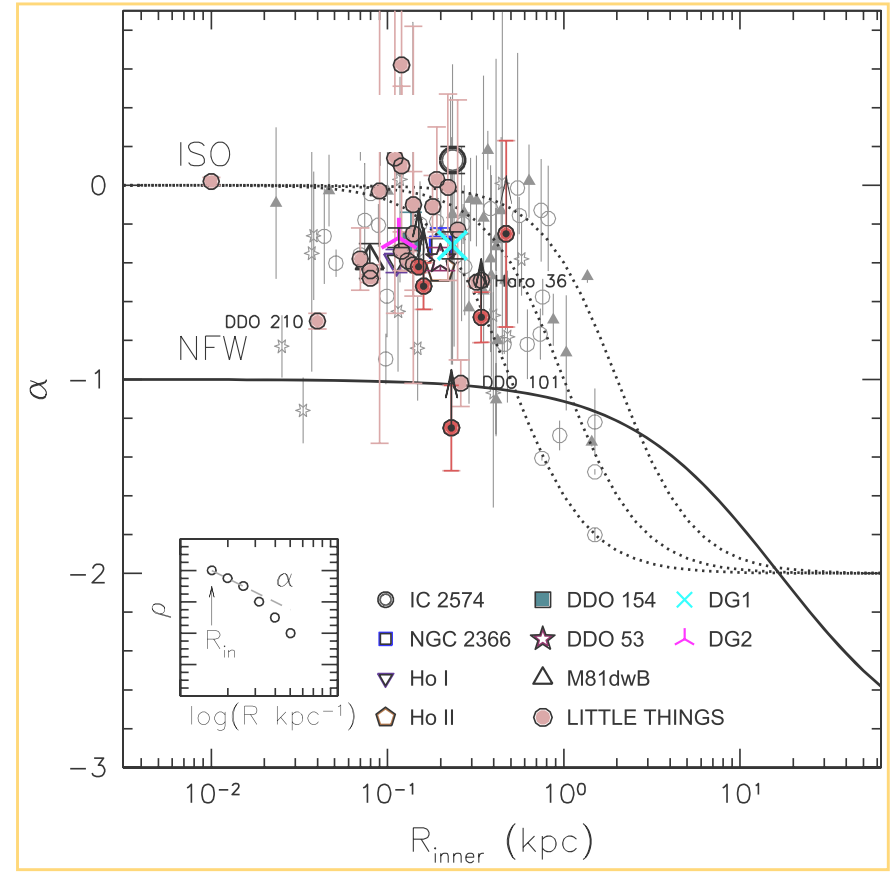

Figure 1. Cusp-core problem: Inner slope $\alpha$ of the density profiles as a function of the radius of the innermost point, within which $\alpha$ is measured. The theoretical slopes of a pseudo-isothermal halo are over-plotted with dotted lines for a core size of 0.5 (leftmost), 1 (centre) and 2 (rightmost) kpc. The solid line represents an NFW model [9]. The pseudo-isothermal model is preferred over the NFW model to explain the observational data (see Equations (1) and (2)). The figure is reprinted from [32].

\subsection{A Promising Solution for Gas-Rich Dwarf Galaxies}

One of the key predictions of the $\Lambda \mathrm{CDM}$ paradigm is that DM assembles into halos that develop cuspy density profiles following the NFW form in the absence of baryonic effects. Indeed, the cusp-core problem in dIrrs was established without the inclusion of baryons. This is the reason that baryonic physics appeared as a natural solution within the $\Lambda \mathrm{CDM}$ framework. Moreover, the size of derived DM cores is typically on the order of a few kpcs, where baryons start to play an important role. Since DM interacts only gravitationally, baryons can affect it through the gravitational potential. The most promising solution, which was designated to explain this discrepancy at small scales, is stellar feedback [33]. This feedback process consists of all interactions of stars with the interstellar medium, which is mostly filled with gas. Contrary to radiative and chemical feedback, this mechanical feedback acts as an energy injection of massive stars in the form of winds or SN explosions [34-38]. Moreover, it was established that, at the dwarf scale, stellar feedback dominates over other feedback processes, such as black hole feedback, as it mainly comes from high-mass stars.

Even if baryons steepen the DM potential well when they cool and accumulate at their centre [39-42], this feedback mechanism is able to alter the DM distribution by generating significant movements of the gas. Indeed, gas gathers in DM halos and feedback can expel large amounts of gas from the bottom of their potential well [33,43-53]. A fraction of this gas then cools and returns to the centre, generating repeated cycles of significant gas outflows, which, in turn, cause rapid fluctuations in the gravitational potential. These potential fluctuations dynamically heat the DM and lead to the formation of a core. As a result, this baryonic process transforms a central DM cusp $(\alpha=-1)$ into a core $(\alpha=0)$. The gradual dispersion of the DM particles away from the centre of the halo is ultimately responsible for core creation. More precisely, these fluctuations in the potential transfer energy into DM particles and expand the DM distribution. Thus, one solution to the cusp-core problem in dIrrs is that a DM heating through stellar feedback generates a cusp-to-core transition for the DM halo within the CDM paradigm. 
Cosmological hydrodynamical simulations performed with different codes such as GASOLINE [54-57], FIRE [58-63], RAMSES [64] and GADGET [65-70] have proven the efficiency of these feedback mechanisms for core creation. Many of the most advanced hydrodynamic simulations with different feedback implementations are able to produce core-like density profiles as inferred from rotation curves, such as those shown in Figure 1. Figure 2, reprinted from [70], shows that, for $\rho_{\text {th }}=0.1 \mathrm{~cm}^{-3}$, the DM profiles are all consistent with NFW form above the convergence radius defined by [71]. For higher values of $\rho_{\text {th }}$, the density profiles depart systematically from NFW in some cases. The dependence of the core radius on the halo mass is highlighted over a wide range of the gas density threshold. This confirms that very-low-mass dwarfs do not exhibit large DM cores as in earlier works [56,57,72]. Moreover, it is also demonstrated that the particular choice of $\rho_{\text {th }}$ determines the size of the core (see Figure 2). However, it was concluded that a value of $\rho_{\text {th }}$ higher than the mean interstellar medium density is necessary for forming cores induced by stellar feedback $[70,73]$. However, these simulations have shown that cores form efficiently only in a narrow range of stellar halo mass, which corresponds to bright dwarf galaxies $\left(M_{*}=10^{7}-10^{9} \mathrm{M}_{\odot}\right)$ (see Figure 2). It was also suggested that the inner slope of DM halos is mass-dependent $[48,56]$. Indeed, a relationship was established between the slope $\alpha$ and the stellar halo mass fraction, $M_{*} / M_{\mathrm{vir}}$, of simulated galaxies [56-59,74]. As a result, there is a characteristic mass ratio of $M_{*} / M_{\mathrm{vir}}=0.005$ for efficient core formation below which DM halos remain similar to the cuspy NFW profile predicted by DM-only simulations. In fact, DM halos become more cored as $M_{*} / M_{\mathrm{vir}}$ increases to this characteristic mass ratio. On the contrary, it was demonstrated that it is possible to induce cusp-to-core transition in dwarfs of all stellar masses [75]. This was made feasible by the fact that the stellar masses of dwarfs were slightly overestimated compared to those of cosmological simulations, such as Illustris TNG [76]. As this gives a good match to observations of dIrrs, it suggests questioning $M_{*} / M_{\mathrm{vir}}$ for dwarf galaxies in our cosmological models.

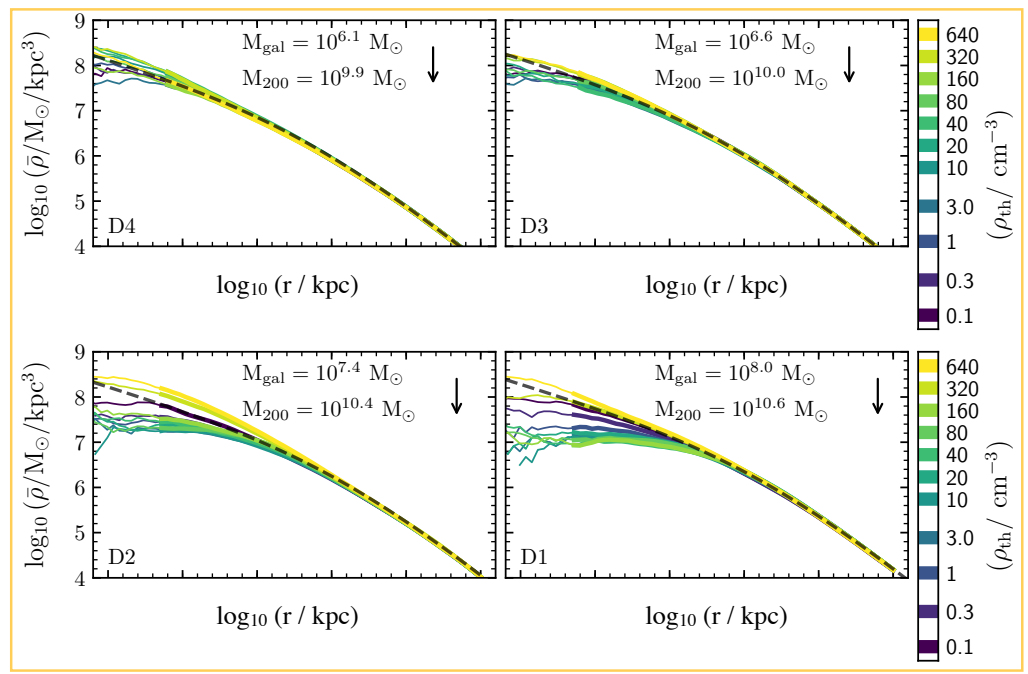

Figure 2. Cusp-to-core transition: Mean enclosed DM density profiles at $z=0$ of four dwarf galaxies with stellar masses between $10^{6}$ and $10^{8} \mathrm{M}_{\odot}$ for different gas density thresholds for star formation, $\rho_{\text {th }}$, compared to the NFW profile (dashed curve) in numerical simulations that incorporate baryonic feedback. The virial radius of the halos is indicated by black arrows. The value of $\rho_{\text {th }}$ varies from 0.1 to $640 \mathrm{~cm}^{-3}$. For $\rho_{\text {th }}=0.1 \mathrm{~cm}^{-3}$, the DM profiles are all consistent with NFW form above the convergence radius defined by [71]. This radius indicates the region within which numerical convergence is not achieved because of two-body relaxation. For higher values of $\rho_{\text {th }}$, the density profiles depart systematically from NFW in some cases. The dependence of the core radius on the halo mass is highlighted over a wide range of the gas density threshold. Very-low-mass dwarfs do not exhibit large DM cores as in earlier works $[56,57,72]$. The particular choice of $\rho_{\text {th }}$ determines the size of the core. This figure is reprinted from [70]. 
Even if hydrodynamical simulations alleviate this $\Lambda$ CDM tension by creating cores, its significance depends on the feedback model [67-70,77]. Indeed, galaxies without sufficient star formation are unlikely to have cores due to the lack of energy from feedback [78]. It was also argued that the timing of star formation relative to DM halo growth can also affect core formation. Cusps can regenerate from the core, induced by the feedback as a result of DM-rich mergers [62]. As discussed, the gas density threshold is a crucial feedback parameter for producing cores in galaxies. Cosmological simulations with low-density thresholds for star formation, such as APOSTLE, Auriga and GEAR [69,79], have been shown to not exhibit DM cores.

\subsection{Review Plan}

This review aims to focus on gas-poor dwarf galaxies with $M_{*}=10^{5}-10^{7} \mathrm{M}_{\odot}$, as the previous solution seems to be only designed for gas-rich dwarfs such as dIrrs, which are still forming stars today. In dSphs, star formation ceased shortly after the beginning of the Universe. In fact, they have characteristically old stellar populations and are generally devoid of gas. All hydrodynamical simulations find that baryonic feedback is negligible in the regime of classical dwarfs $\left(M_{*} / M_{\mathrm{vir}}<10^{-4}-10^{-3}\right)$, as expected on energetic grounds $[78,80]$. Thus, it seems more and more challenging to find solutions without invoking baryonic processes as DM cores tend to persist even for these dwarfs, which are rather DM-dominated. In the absence of new solutions in $\Lambda$ CDM, it will inevitably be necessary to directly question the nature of the DM to reproduce the observations at small scales in these galaxies.

Although the CDM paradigm can successfully explain various observations at different scales, this discrepancy at small scales remains one of the greatest challenges faced by this DM theory (see [81] for a detailed review on the observational challenges and see [72,82-84] for global reviews related to the cusp-core problem). Even if we focus only on the cusp-core problem in this review, there are other tensions of the $\Lambda$ CDM model at small scales, which are the missing satellites problem, the too big to fail problem and the alignment of the substructures in the Galactic halo [72,85-87]. This review is intended to give an overview of the current observational and theoretical status concerning the DM distribution at small scales for gas-poor dwarf spheroidal galaxies but also seeks to offer new directions to solve this challenging problem.

\section{The Cusp-Core Problem in Gas-Poor Milky Way Satellites}

Close to the MW and M31, one finds predominantly dwarf spheroidals. These dwarfs are among the most DM-dominated galaxies in the Universe [88,89]. As DM constitutes $90 \%$ or more of the total mass in these dwarf spheroidals, the dynamics are determined entirely by the gravitational field of the DM. Therefore, these systems provide an excellent laboratory to study DM distribution at small scales. The eight most common dwarf spheroidals are the galaxies orbiting around our galaxy and also named "classical" dwarfs. These dSphs have a stellar component of around $10^{6} \mathrm{M}_{\odot}$ embedded in a DM halo of around $10^{9} \mathrm{M}_{\odot}$ (see Table 1). As depicted in the table, the DM masses are poorly constrained. Its estimate is limited to two observed values: the line-of-sight velocity dispersion and the projected half-light radius. Moreover, we underline that only one measurement of the line-of-sight velocity dispersion per galaxy is available for the MW dwarfs. 
Table 1. Classical dwarf spheroidal galaxies: From left to right, the columns give, for each gas-poor dwarf: the galaxy stellar mass from [90] assuming a mass-to-light ratio of 1, the DM mass from [91], the stellar-to-halo mass ratio and the the number of kinematic member stars from [92]. We have chosen to show the DM mass of the dwarfs assuming the presence of a core in order to underline that these systems are even more dominated by DM following this density profile. The ratios calculated here are only intended to give an idea of the scale in regard to the uncertainties on the DM masses.

\begin{tabular}{ccccc}
\hline Dwarf & $\mathbf{M}_{*}\left[\mathbf{1 0}^{\mathbf{6}} \mathbf{M}_{\odot}\right]$ & $\mathbf{M}_{\mathbf{v i r}}\left[\mathbf{1 0}^{\mathbf{9}} \mathbf{M}_{\odot}\right]$ & $\mathbf{M}_{*} / \mathbf{M}_{\mathbf{v i r}}\left[\mathbf{1 0}^{-\mathbf{4}}\right]$ & Member Stars \\
\hline Fornax & $14 \pm 4$ & $2.5_{-1}^{+22}$ & 56 & 2573 \\
Leo I & $3.4 \pm 1.1$ & $25_{-0}^{+6}$ & 1.3 & 328 \\
Sculptor & $1.4 \pm 0.6$ & $25_{-20}^{+14}$ & 0.5 & 1351 \\
Leo II & $0.59 \pm 0.18$ & $25_{-24}^{+14}$ & 0.23 & 186 \\
Sextans & $0.41 \pm 0.19$ & $0.4_{-0.27}^{+0.39}$ & 10.25 & 417 \\
Carina & $0.24 \pm 0.1$ & $2.0_{-1.8}^{+37}$ & 1.2 & 767 \\
Ursa Minor & $0.20 \pm 0.09$ & $25_{-20}^{+14}$ & 0.07 & 430 \\
Draco & $0.27 \pm 0.04$ & $25_{-15}^{+14}$ & 0.1 & 504 \\
\hline
\end{tabular}

\subsection{Dynamical Models}

As most dwarf galaxies are devoid of gas, it is necessary to look at the kinematics of their stars in order to probe their DM inner region. Indeed, rotation curve measurements are impossible for $\mathrm{dSphs}$ as they lack rotating gas components. However, only line-of-sight velocities of stars are observable. The line-of-sight velocity dispersion of these stars from the spherical Jeans equation $[93,94]$ can be written as [95]:

$$
\sigma_{\text {los }}^{2}=\frac{2}{\sum_{*}(R)} \int_{R}^{\infty}\left(1-\beta \frac{R^{2}}{r^{2}}\right) \frac{v(r) \sigma_{\mathrm{r}}^{2}(r) r}{\sqrt{r^{2}-R^{2}}} \mathrm{~d} r,
$$

where $\Sigma_{*}(R)$ is the surface mass density at projected radius $\mathrm{R}$ and the radial velocity dispersion $\sigma_{\mathrm{r}}^{2}(r)$ is defined as:

$$
\sigma_{\mathrm{r}}^{2}(r)=\frac{1}{v(r) g(r)} \int_{r}^{\infty} \frac{G M(u) v(u)}{u^{2}} g(u) \mathrm{d} u,
$$

with

$$
g(r)=\exp \left(2 \int \frac{\beta(r)}{r}\right),
$$

where $v(r)$ and $\beta(r)$ are the radial density profile and the velocity anisotropy, which describes the orbital structure of the stellar system, respectively. $\beta=0,1$ and $-\infty$ correspond to an isotropic, fully radial and fully tangential distribution, respectively.

This technique allows the measurement of the central DM density profile in galaxies as the line-of-sight velocity dispersion of stars depends on the mass profile $M(r)$ [96-98]. However, there is a degeneracy between the radial density profile of DM, $\rho(r)$, and the unknown orbit distribution of the stars. This latter is typically characterized by the velocity anisotropy parameter $\beta$, which is hard to constrain with only line-of-sight velocities $[95,97,99,100]$. This is the reason that analyses of the line-of-sight velocities in dwarf galaxies have led to contradictory conclusions. Some authors conclude that the kinematic data require DM core [101-103], while others found that the data are also consistent with the NFW form [104-106]. For the brighter MW dwarfs, this degeneracy can be broken by using metallicity or colour to split the stars into distinct components $[102,103,107]$. Other methods have been proposed to break this degeneracy by using higher-order velocity moments [108], Schwarzschild methods [105,109] and proper motions [110-112]. Indeed, together with line-of-sight velocities and positions on the sky, stellar proper motions, which are the two additional transverse velocity components, provide five out of the six phase-space coordinates of the stars. The degeneracy may be also broken by including the fourth-order projected virial theorem [99]. A non-parametric Jeans method, namely GravSphere, em- 
ploys the additional constraints from the virial shape parameters in the analysis [97]. This higher-order Jeans analysis method has been shown to successfully recover the DM density distributions of simulated dwarfs above half of the projected half-light radius [97,98]. Incorporating proper motions of stars was also employed to ameliorate this mass-anisotropy degeneracy $[111,113]$.

In Figure 3, the DM density profile of eight dwarf spheroidal galaxies was estimated by using stellar kinematics [92]. In the $68 \%$ confidence interval, it is hard to distinguish between cusp and core for the MW satellites according to GravSphere fits (see Figure 3). As their profile is better constrained at a radius of $150 \mathrm{pc}$, it was established that seven dwarfs have a central DM density $\rho(150 \mathrm{pc})$ consistent with a cusp and only Fornax had a $\rho(150 \mathrm{pc})$ consistent with a DM core [92]. However, this still leaves room for DM cores of less than 100 pc based on GravSphere model uncertainties (see Figure 3). Accounting for dwarfs in dynamical equilibrium, Ref. [114] found also a diversity of DM density profiles, with many actually favouring cuspy profiles.

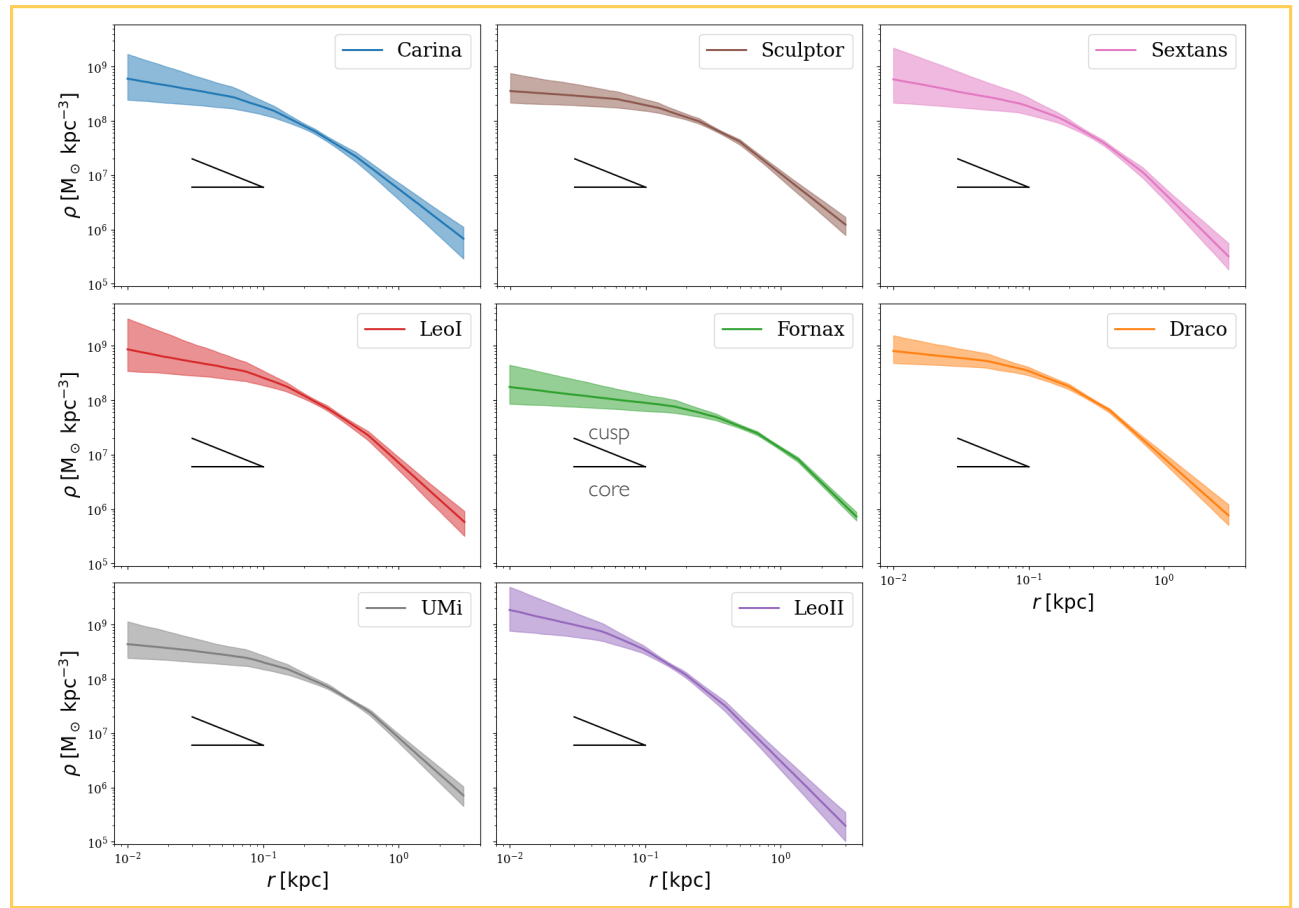

Figure 3. Dynamical modeling: DM density profiles of the eight MW classical dwarf galaxies derived from the stellar kinematics [92]. The shaded regions mark the $68 \%$ confidence intervals of the model. In this interval, it is hard to distinguish between cusp and core for the MW satellites according to GravSphere fits.

Furthermore, the Jeans method usually assumes dwarfs as spherical systems for simplicity. However, it was claimed that the stellar component of the dwarfs is actually non-spherical $[17,115]$. As they formed in a hierarchical manner, DM halos are also expected to be non-spherical [116-118]. Ref. [119] applied the Jeans technique to the line-of-sight velocity dispersion profiles of seven MW dwarfs. Contrary to [92], they found that five dwarfs, including Fornax, have a cored central density profile [119]. Indeed, non-spherical halo models seem to reveal a more diffuse DM distribution in the inner region of dwarfs. By using the Jeans modelling, it is generally assumed that the MW tides have not had much impact on the stellar kinematics of dwarfs. However, there is a different physical effect, which is not due to tidal stripping and occurs only for highly eccentric orbits, namely tidal shocking [120-122]. In fact, the MW tidal shocks can bring sufficient kinetic energy to heavily affect the velocity dispersions of stars. Since DM calculations are based on stellar kinematic measurements, one may wonder whether they could have been corrupted by the fact that $\mathrm{dSphs}$ were out of equilibrium because of MW tides $[90,123,124]$. It was 
demonstrated that it takes more than three dynamical times for a system to virialise after a perturbation [125]. Finally, this questions the validity of the dynamical mass estimate using the Jeans equation, and hence of the estimates of the DM amount in MW dwarfs.

Moreover, a recent study highlights the need for a large number of kinematic member stars for dwarfs in order to accurately determine the DM inner profile [126]. By using mock observations, the authors showed that it is necessary to measure approximately 10,000 stars within a single dwarf galaxy to infer correctly the DM distribution at small scales. With data sets of fewer than 10,000 stars, it appears that the DM density distribution is biased towards a steeper inner profile than the true distribution by applying the Jeans method. This effect could explain why [92,114] found that the majority of classical dwarfs exhibit cuspy profiles. As described in Table 1, the number of stars used by [92] to infer the DM profiles of MW dwarfs is well below what is recommended by [126].

Despite the complexity of the Jeans analysis, dynamical models are often claimed to require shallower density profile slopes that are consistent with a core at their centre [92,102,103,127,128]. The mass measurements of [129] reinforce the prediction for the presence of DM cores in gas-poor MW satellites. Figure 4, reprinted from [61], shows the circular velocity of 19 subhalos in the DM-only simulation at $z=0$. Only five subhalos from the GIZMO cosmological simulation are consistent with Ursa Minor, Draco, Sculptor, Leo I and Leo II. As a result, the other DM subhalos are too dense. One way to reduce the inner DM density of halos is core formation.

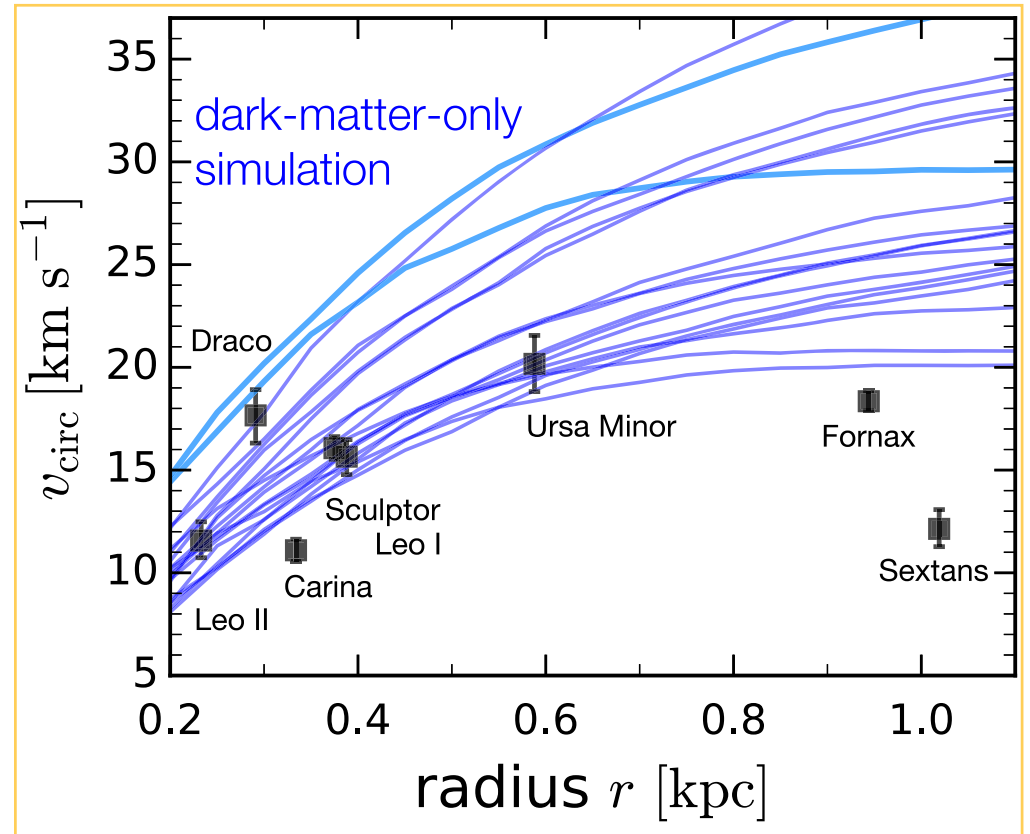

Figure 4. Rotation curves of classical dwarfs: Circular velocity of 19 subhalos in the dark-matter-only simulation at $z=0$. Black points show observed gas-poor satellites of the MW [129]. Only five subhalos from the GIZMO cosmological simulation are consistent with Ursa Minor, Draco, Sculptor, Leo I and Leo II. This figure is reprinted from [61].

\subsection{Controversy on Producing DM Cores via Supernova Feedback}

Historically, stellar feedback was invoked to solve this issue. However, as described by Table 1, the classical dwarfs, except Fornax, are in the mass ratio regime $\left(M_{*} / M_{\text {vir }}<10^{-4}-10^{-3}\right)$, where the contribution of feedback for creating a DM core is negligible $[57,78,80,130]$. In addition, only Fornax has extended star formation compared to the other dwarfs [92]. This is the reason that stellar feedback via supernovae explosions could explain the presence of a DM core as star formation proceeds for long enough in Fornax [92]. Nevertheless, star formation shut down 1.75 Gyr ago in Fornax and it was demonstrated that, in $10^{9} \mathrm{M}_{\odot}$ dwarfs such as Fornax, DM cores induced by multiple repeated bursts need $\sim 14$ Gyr to 
fully form [75]. The efficiency of this core creation mechanism in dwarf galaxies remains under intensive debate in the literature. We detail the possible reasons for the disagreement between isolated and cosmological hydrodynamic simulations.

By using idealized high-resolution simulations, Ref. [75] argued that DM cores form if star formation proceeds for long enough, but this gives stellar-to-halo mass ratios, which are not obtained in CDM cosmological simulations in the dwarf regime $[56,57,59,69,130,131]$. It was also reported that in the non-cosmological simulation of [75], some missing ingredients such as the UV background and the halo growth via mergers could be the most important sources of the differences. Indeed, the strong, ionising UV background radiation has been identified as being capable of evaporating most of the gas in dwarf galaxies [132-135]. In addition to the fact that cosmological simulations of galaxy formation tend to be more realistic than isolated simulations, the ingredients for galaxy formation are calibrated to the resulting structural properties of observed massive galaxies. As the efficiency of this cusp-to-core mechanism at dwarf scale is mainly determined by the gas density threshold for star formation, as discussed before, core formation thus depends on the baryon physics implemented in the simulation [70,73]. However, in some studies that have claimed the absence of DM cores in dwarfs, it was stressed that they are unable to resolve the clumpy interstellar medium, which is crucial for observing cusp-core transformations via supernova feedback [48,92]. Recently, Ref. [136] pointed out that supernova feedback is a feasible mechanism of cusp-core transformation in dwarfs only if the supernova energy injection is longer than the dynamical timescale of DM particles in the inner halo. Moreover, they also stressed that DM heating is more efficient if baryons are more concentrated towards the centre of the galaxy [136]. Previous hydrodynamical simulations established a seeming connection between the presence of DM cores and the star formation history of dwarf galaxies [75], but there is a consensus that finding signatures of stellar feedback is not a sufficient condition for dwarfs to exhibit cores [136]. Indeed, baryon-induced cores in dwarfs would be difficult to distinguish from DM cores produced by other mechanisms, such as in alternative DM theories [137-139].

Cosmological simulations have to cover a wide range of spatial and timescales. It is challenging to capture all relevant scales for this type of simulation. This is the reason that dwarf galaxies continue to be one of the few areas where the CDM cosmological model has difficulties matching observations. IllustrisTNG, as one of the most recent cosmological hydrodynamical simulations, has achieved a mass and a spatial resolution of $\sim 10^{6} \mathrm{M}_{\odot}$ and $0.2 \mathrm{kpc}$, respectively [76]. This main limitation has been pointed out as the source of the inconsistencies between predictions made by the CDM paradigm and observations. This also contributes to explaining why the implementation of star formation and feedback is challenging. Moreover, it is imperative to remember that the problem of star formation is still unsolved at all redshifts and totally unconstrained at high redshift. Even non-cosmological simulations require a number of choices and assumptions about the initial conditions; as well as the input physics, it is essential to investigate the small-scale physics in dwarf galaxies and test various mechanisms. Idealised simulations must make a contribution. Thanks to these zoom simulations, we can achieve spatial resolutions of up to $0.03 \mathrm{kpc}$ with the VELA hydrodynamical simulation [140] and mass resolution up to $6.2 \times 10^{2} \mathrm{M}_{\odot}$ with the NIHAO hydrodynamical simulation [74]. However, this limitation should soon be overcome by extreme-resolution simulations [141], allowing us to probe smaller physical scales than previously possible in cosmological simulations. These simulations, with a mass and spatial resolution of $30 \mathrm{M}_{\odot}$ and $\sim 0.1-0.4 \mathrm{pc}$, predict that the stellar do not significantly alter the density profile from cuspy to cored distribution [74]. This result is consistent with some of the lower-resolution cosmological simulations [62,78]. However, feedback still needs to be modelled properly at these resolved scales.

\section{Solutions}

In this section, we investigate some of the most popular and promising solutions to the cusp-core problem. We are particularly interested in solutions that could replace stellar feedback. Indeed, this core mechanism seems inefficient for most dSphs, such 
as the gas-poor MW satellites. There are two main approaches that could solve this discrepancy between $\Lambda \mathrm{CDM}$ and observations. Cosmological solutions invoke a different spectrum at small scales [142], different nature for DM particles, such as fuzzy and selfinteracting DM [143-148], modified gravity theories [149-157] or Modified Newtonian dynamics [158-161]. On the contrary, astrophysical solutions invoke sub-galactic baryonic physics within the $\Lambda$ CDM paradigm, such as stellar feedback [33]. A common aspect of these two broad categories of solutions is that core creation has been identified as their main mechanism. In this review, we adopt a different classification. Our first category includes scenarios where DM cores emerge due to the flattening of initial $\Lambda C D M$ cusps, named cusp-to-core solutions. We then focus on solutions that depict the natural emergence of DM cores, such as in fuzzy and self-interacting DM theories, named inherent core solutions.

\subsection{Cusps to Cores}

It is admitted that a massive particle moving through an infinite, homogeneous and isotropic background of lighter particles experiences a force of dynamical friction given by

$$
F(x, v)=2 \pi G^{2} \rho(x) \ln \left(1+\Lambda^{2}\right)\left(\operatorname{erf}(X)-\frac{2 X}{\sqrt{\pi}} \exp \left(-X^{2}\right)\right) \frac{v}{|v|^{3}} M,
$$

where this massive particle of mass $M$ at position $x$ is moving at velocity $v$ through a background density $\rho$ [162]. The quantity $X$ is defined as $|v| / \sqrt{ }(2) \sigma_{\mathrm{r}}$, with $\sigma_{\mathrm{r}}$ being the radial dispersion of lighter particles. The factor $\Lambda$ that goes into the Coulomb logarithm is taken to be

$$
\Lambda=\frac{r / \gamma}{\max \left(r_{\mathrm{hm}}, G M /|v|^{2}\right)}
$$

where $r_{\mathrm{hm}}$ is the half-mass radius of the massive particle and $\gamma$ is the absolute value of the logarithmic slope of the density, i.e., $\gamma=|d \ln (\rho) / d \ln (r)|$ [163]. The background medium composed of lighter particles produces an overdensity region behind it due to this friction between particles. The dynamical friction is responsible for a momentum loss by the massive object due to its gravitational interaction with its own gravitationally induced wake. The surrounding background medium, which consists of a combination of collisionless matter such as DM, is heated at an equal and opposite rate to the energy lost by the massive object. The rate of energy loss by the massive object is given by [164]:

$$
\frac{\mathrm{dE}}{\mathrm{d} t}=M \frac{\mathrm{d} v}{\mathrm{~d} t} v
$$

An energy exchange occurs, increasing that of the medium particles at the expense of the perturber. If the perturber passes close to the central region of a dwarf galaxy, it could modify the DM inner structure via dynamical friction. During the perturber infall within the galaxy, it transfers part of its kinetic energy to the DM background through dynamical friction, causing the DM particles in the central region of dwarfs to migrate outwards. The particle heating and migration in the central region of the galaxy is expected to lead to the flattening of the DM density profile. Indeed, at kpc scales, this collective effect induces potential fluctuations, which erode the central density cusp of the DM halo.

\subsubsection{Mergers with Dwarf Galaxies}

In our cosmological model, galaxies form in a hierarchical manner. They are formed, on the one hand, by mergers of pre-existing galaxies. High-resolution $N$-body simulations have shown that, as the satellite falls onto M31, it is slowed down by dynamical friction and its energy is transferred to the host halo. In this process, the initial cusp shallows down for over a decade and is well-fitted by a core profile [165]. The efficiency of this mechanism depends on the mass, as depicted by Equation (6), and on the orbit of the perturber. Indeed, it was suggested that the cusp-to-core transition occurs where the mass of the perturber 
within its tidal radius $r_{\mathrm{t}}$ roughly matches the enclosed mass of the DM background as follows:

$$
M_{\text {pert }}\left(r_{\mathrm{t}}\right) \sim M_{\mathrm{DM}}\left(r_{\mathrm{p}}\right),
$$

where $r_{\mathrm{p}}$ is the instantaneous orbital radius of the perturber [166,167]. Moreover, it has been reported that merger events in which satellites fall on highly eccentric orbits onto their host halos can initiate core formation in a $\Lambda$ CDM Universe where halos have cuspy profiles [165]. In order to alter the DM distribution by scattering particles away from the centre, the satellite needs to pass through the central region of the galaxy. This condition is only satisfied with nearly radial orbits.

Now, the question is to determine whether the cusps of the dwarf galaxies could be disrupted during mergers. Major mergers of dwarf galaxies are very rare after $z \sim 3$ [168]. However, the CDM paradigm predicts that a very large number of DM substructures exist inside galactic halos $[169,170]$. Recently, Gaia DR2 data have provided additional evidence for these substructures [171]. DM halos are growing with time, notably by the accretion of smaller halos, considered DM subhalos. They interact gravitationally with all the components of the galaxy before becoming remnants of disrupted halos [172]. It was pointed out that $10^{9}\left(10^{10}\right) \mathrm{M}_{\odot}$ dwarf halos have accreted 10-11 (13-14) subhalos with a mass ratio $10<M_{\text {host }} / M_{\text {sub }}<100$ over their history [173]. This can be seen as minor mergers with these subhalos. Moreover, based on the approximated orbital distributions of satellites by [174], it was shown that subhalos exhibit orbits, which are nearly radial with an eccentricity $e=0.85(e=0.88)$ at $z=3$ for $10^{9}\left(10^{10}\right) \mathrm{M}_{\odot}$ dwarf halos [173]. Thus, dynamical perturbations induced by subhalo crossings, causing black holes (BHs) to vacate the galaxy centre, could also modify the spatial distribution of DM particles [173]. Nevertheless, subhalos possess a very diffuse DM distribution. This is the reason that the condition described by Equation (9) is satisfied only for small radii. Ref. [173] demonstrated that the maximum offset reached by the $\mathrm{BH}$ due to heating from subhalos is $134 \mathrm{pc}$, which delimits the region where the DM distribution could have been significantly perturbed by subhalos. It was recently shown that, in ultra-faint dwarf galaxies, the potential fluctuations could be also due to subhalo crossings, but the DM distribution remained cuspy while it was flattened [175]. It is possible that the combination of stellar feedback and subhalos could then enhance the flattening of the central DM density [175]. This mechanism seems unfortunately inefficient, particularly in the case of the Fornax dwarf, which requires a DM core of size $\sim 1 \mathrm{kpc}$ (see Figure 3). Recently, it was suggested that a dwarf major merger is needed to recover the current spatial distribution of globular clusters (GCs) in Fornax [176]. This ancient merger ( $\sim 10$ Gyr ago) could have contributed to the formation of the large DM core in the Fornax dwarf.

Moreover, it was claimed that the stellar component of the satellite play a major role in core formation. Indeed, as this component is more concentrated compared to the DM of the satellite $\left(a_{*} / a_{\mathrm{DM}}=0.1\right)$, it will further slow down the satellite during its infall and thus disturb the central region of the host even more prominently [165]. As the stellar component of the satellite enhances the destruction of the cusp, galaxies with a low halo-to-stellar ratio could be promising candidates for such minor mergers, but they are only found at very high redshift.

\subsubsection{Globular Clusters and Gas Clumps}

Galaxies also grow by the accretion of a variety of objects, such as GCs and gas clumps. This is the reason that such massive objects have also been proposed for transforming cusps into cores via heating by dynamical friction [164,166,177-180]. Figure 5, adapted from [166], shows the modification of the inner DM structure after the first (upper panel) and second (lower panel) closest pericentre passage of massive objects such as gas clumps or GCs with different masses. The perturbers were started within the cusp region. All simulations are shown using circular orbits for the infalling objects. We note that the response of different central cusps to sinking perturbers with a range of masses using $N$-body simulations occurs rapidly. The DM density distribution changes significantly from cuspy to having a core. 
Indeed, larger perturber masses lead to larger constant density central regions, as predicted by Equation (9).

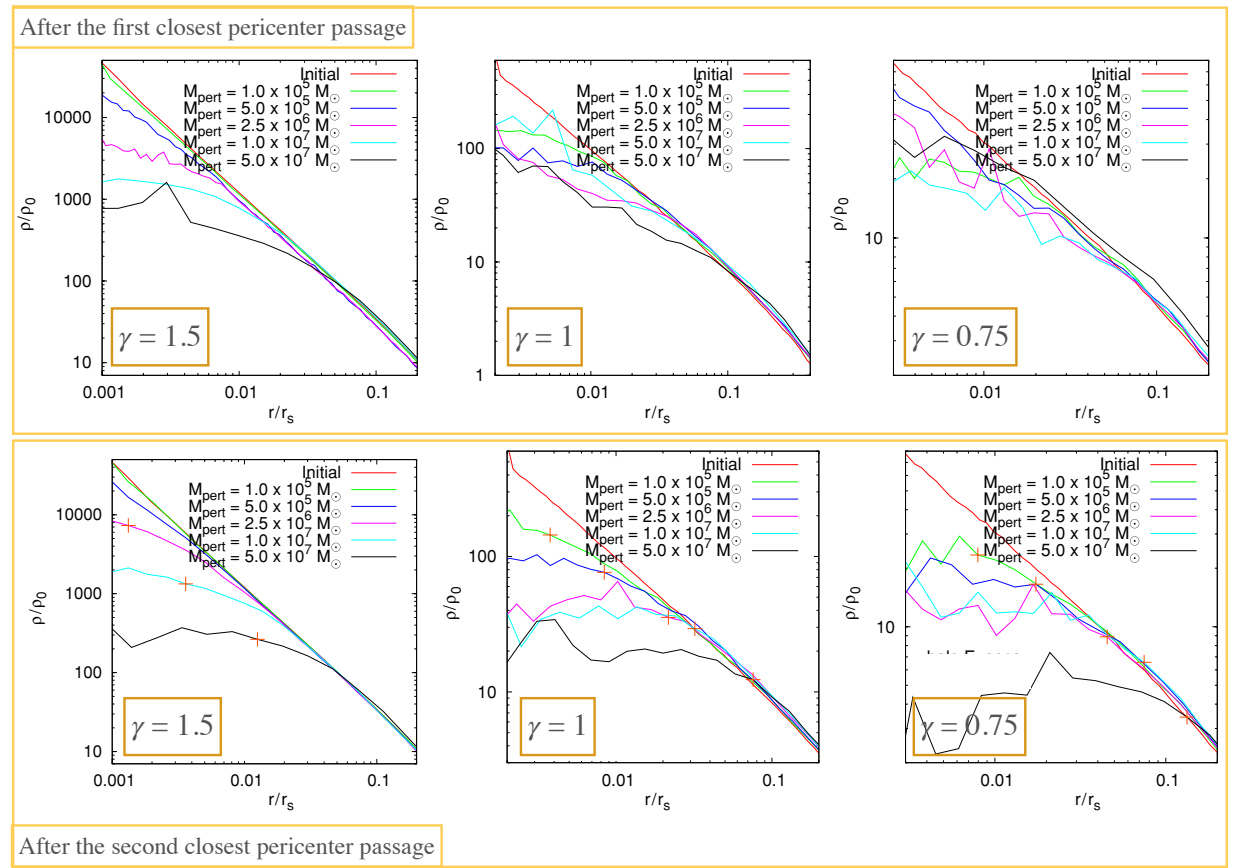

Figure 5. Sinking of massive objects: DM density profiles of the host halo after the first (upper panel) and second (lower panel) closest pericentre passage for the different perturber masses $M_{\text {per }}=$ $\left[10^{5}, 5 \times 10^{5}, 2.5 \times 10^{6}, 10^{7}, 5 \times 10^{7}\right]$. From left to right, the panels show halos with different initial absolute values of the logarithmic slope of the density $\gamma=|\mathrm{d} \ln (\rho) / \mathrm{d} \ln (r)|$. The DM density distribution changes significantly from cuspy to having a core. This figure is adapted from [166].

The results of these works clearly indicate, as a proof of concept, that dynamical friction heating can have an important role in DM halos on different scales and the relevance of this process depends on the properties of the massive objects and of the host halo. Such a mechanism still requires another process to then destroy the gas clumps and GCs at the centre of the DM halo. Otherwise, the resulting inner stellar density would be too high to be consistent with observations [177]. For the gas clumps, stellar feedback could dissolve these clumps. However, GCs form nuclear star clusters at the centre of galaxies, but observations claim that none of the classical dwarfs exhibit a stellar nucleus at their centre.

\subsubsection{Globular Clusters Embedded in Dark Matter}

GCs are gravitationally bound groupings of mainly old stars, formed in the early phases of galaxy formation. Classically, it has been claimed that GCs do not contain DM because their dispersion velocities are too small. However, these measures are done at the centre of GCs, where the influence of DM is very small. An absence of evidence is not evidence for absence. Currently, there is no clear consensus on the formation of GCs, a subject that is hotly debated and brings unique constraints on the formation of small-scale halos in the $\Lambda$ CDM paradigm of galaxy formation. It has been proposed that GCs may have a galactic origin, where GCs are formed within DM minihalos in the early Universe [181-184]. Then, these GCs could have merged to become, later, a part of the present-day host galaxy. Until now, these DM halos have not been detected. More precisely, it was pointed out that the ratio of the mass in DM to stars in several GCs is less than unity [185-191]. Even if GCs are proven not to have a significant amount of DM today, it does not preclude them from having been formed originally within a DM minihalo. A natural explanation is that they have lost their DM over time. Indeed, there are several internal dynamical processes that could eject DM from GCs, such as DM decay [192] and feedback processes [48,193]. It was also shown that GCs orbiting in the inner regions of their host galaxies may lose a large fraction of their primordial DM minihalos due 
to tidal stripping $[182,183,194,195]$. This is the reason that the main mechanism by which most GCs could have lost their DM minihalo is through severe tidal interactions with our galaxy given their current positions. Nevertheless, GCs at a large distance from the MW centre could have retained a significant fraction of DM because it was not completely stripped by the galaxy. Even if observations of these GCs, such as NGC 2419 and MGC1, highlight that they do not possess significant DM today $[185,186]$, it does not exclude the existence of DM in GCs but suggests that there is not necessarily a unique formation mechanism for GCs.

The motion of GCs embedded in DM minihalos inside the CDM halo of Fornax was studied by considering both early and recent accretion scenarios of GCs by Fornax, with the most prevalent initial conditions taken from Illustris TNG-100 cosmological simulations [196]. Using high-resolution simulations, these minor mergers involved perturbers with a low halo-to-stellar ratio ( 10-20), which make GCs more massive. This is why they fall more rapidly towards the galaxy center [197]. As expected, GC crossings near the Fornax centre induce a cusp-to-core transition of the DM halo. Moreover, if the five GCs were accreted recently, less than 3 Gyr ago, by Fornax, they should still be in orbit and no star cluster should form in the centre of Fornax, in accordance with observations [197]. By designing initial conditions such as GC orbits outside the Fornax tidal radius, avoiding the formation of a nuclear star cluster at the Fornax centre is possible without invoking this new dark component [198]. Nevertheless, crossings of GCs with a DM minihalo near the Fornax centre induce a cusp-to-core transition of the DM halo and hence resolve the cusp-core problem in this dwarf galaxy. The DM core size depends strongly on the frequency of GC crossings [197]. It was subsequently highlighted that an infalling GC with a DM minihalo enhances core formation without forming a nuclear star cluster at the Fornax centre. Moreover, their results are in good agreement with the constraints on the DM mass of GCs as these clusters lost a large fraction of their DM minihalos. All of these aspects provide circumstantial evidence for the existence of DM halos in GCs. Nevertheless, it was pointed out that this should be regarded as unlikely since GCs do not appear to be ubiquitous in local dwarf galaxies [197].

\subsubsection{Tidal Interactions}

It is well-known that the classical dSph galaxies are satellites of the MW. Studies about dwarfs of the Local Group have revealed that DM cores can be generated through tidal stripping $[91,199,200]$. By removing more and more bound particles, in an outside-in fashion, the effect of tides was also proposed as a solution to the cusp-core problem in a CDM universe. Indeed, the mass removal due to tidal forces can reduce the DM content at all scales, even in the central region [201]. This alternative mechanism was tested by [202] in order to explain the low inferred density in Fornax (see Figure 3). The majority of their Fornax analogues are able to lose DM from the inner $1 \mathrm{kpc}$ due to tidal effects [202]. Even if this mechanism leads to a reduction in the DM density at all radii, the inner DM region of Fornax remains cuspy. Ref. [201] also stressed that there is a steepening of the central slope of the DM profile during satellite accretion by a MW-like galaxy, even if dwarfs, which have shallower DM profiles due to feedback heating before accretion, evolve into cuspy DM halos [201]. Thus, due to a low orbital pericentre in the MW or due to tidal interactions with other galaxies prior to infall, Fornax could exhibit a cuspy DM halo, with its low density owing entirely to tides (see Figure 3). However, the absence of a DM core profile is still in tension with the kinematics of Fornax below its half-light radius, depicted in our Table 1 [92]. Furthermore, there is currently no sign of tidal stripping in Fornax. In other words, no stream of unbound stars has yet been detected $[203,204]$.

\subsubsection{Cusp Regeneration}

Along with mechanisms that flatten the central DM density, there are mechanisms that can rebuild it. Even if feedback processes can generate cores in DM halos, simulations of dwarf galaxies have shown that a DM cusp could regenerate in the centre of the halo [62,205]. It was claimed that the infall of substructures such as minor mergers with cuspy halos is responsible for this cusp regrowth $[59,62,175,205,206]$. Figure 6 illustrates the cusp 
regeneration of a DM halo at late times due to the merger with a cuspy satellite. In the same way, it was shown that the passages of DM minihalos of GCs could significantly perturb the DM distribution in the Fornax halo centre [197]. Indeed, between crossings, the halo can reform the cuspy halo owing to the new orbits of DM particles initially at the Fornax centre as they gained energy from the GCs. In the right panel of Figure 6, we observe reverse transitions of the Fornax DM halo. More precisely, there are forward and reverse transitions from the cusp to the core [197]. We argue that DM minihalos, which are still orbiting in the host dwarf, induce potential fluctuations and then displace the DM potential centre. This potential shift is responsible for the cusp regeneration as these subhalos are much denser. As shown before, tidal interaction with a host galaxy can also contribute to core-cusp transformations. Ref. [207] stressed that it is questionable whether DM cores in classical dwarfs could subsequently survive to the present day without being tidally disrupted by the MW. As such, the cuspy profile seems to be more common at recent epochs, as predicted by [92]. However, it is unclear on which timescales this process is more likely to occur as it depends on the merger history and on the environment. As there is a cusp regrowth problem within CDM, this leaves open the question of whether cores are only transient states. Therefore, we should expect to observe a diversity of DM profiles at a given mass. Ref. [92,119] found that our local dwarf galaxies can be separated into two distinct classes, those with cold DM cusps and DM cores (see also Figure 3). This transient phenomenon could explain this diversity in the dwarf regime.

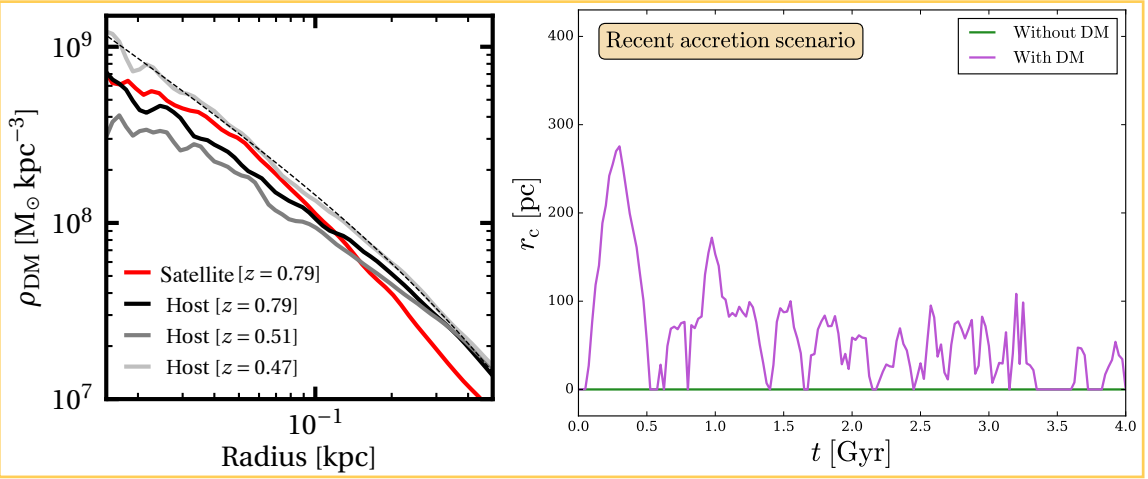

Figure 6. Cusp regeneration in dwarfs: Left panel: DM density profiles of a cuspy satellite (red line) and of the host halo at different redshifts (black, dark grey and light grey lines). A black dashed line represents an NFW profile fit to the host halo at $z=0.79$. The merger with the satellite reforms the erased cusp. Right panel: Fitted core radius $r_{\mathrm{C}}$ of the DM halo induced by crossings of GCs with (in purple) and without (in green) a DM minihalo as a function of time. $r_{\mathrm{C}} \neq 0\left(r_{\mathrm{c}}=0\right)$ means that there is (no) cusp-to-core transition for the dwarf DM halo. This figure is adapted from $[175,197]$.

\subsubsection{The Diversity Problem}

A key observable related to the inner mass distribution of galaxies is their rotation curve. The circular velocity curves of simulated galaxies vary systematically as a function of their maximum circular velocity $V_{\max }$, with a marginal uncertainty according to the CDM model. On the other hand, observed galaxies show a large diversity of rotation curve shapes, even at fixed maximum rotation velocity, especially for dwarf galaxies. This is at odds with the expectation for CDM halos, where $V_{\max }$ fully determines $V_{\text {circ }}(2 \mathrm{kpc})$, and this has been termed the diversity problem [77]. The origin of this diversity is still not well understood.

Figure 7 shows the circular velocity $V_{\text {circ }}(2 \mathrm{kpc})$ versus $V_{\max }$ for observed galaxies. We used the coreNFW model in order to characterise the inner DM density from these observed circular velocities. The coreNFW profile is a fitting function, which captures the cusp-core transformation [75]. For this model, the cumulative mass profile is given by:

$$
M_{\mathrm{CNFW}}(<r)=M_{\mathrm{NFW}}(<r) f^{n},
$$


where $M_{\mathrm{NFW}}$ is the NFW mass profile and $f^{n}$ generates a shallower density profile below a core radius $r_{\mathrm{c}}$ :

$$
f^{n}=\left[\tanh \left(\frac{r}{r_{\mathrm{c}}}\right)\right]^{n},
$$

where the parameter $0<n \leq 1$ controls how shallow the core becomes and corresponds to the transition region between cusp and core. Indeed, $n=0(n=1)$ corresponds to a fully cuspy (core) halo. The density profile of the coreNFW model is given by:

$$
\rho_{\mathrm{cNFW}}=f^{n} \rho_{\mathrm{NFW}}+\frac{n f^{n-1}\left(1-f^{2}\right)}{4 \pi r^{2} r_{\mathrm{C}}} M_{\mathrm{NFW}}
$$

Given the halo mass and redshift, both halo concentrations $c_{200}$ can be estimated from cosmological $N$-body simulations. Indeed, the mass and concentration of halos at redshift $z=0$ in $\Lambda C D M$ are correlated:

$$
\log _{10}\left(c_{200}\right)=0.905-0.101 \log _{10}\left(M_{200} h-12\right)
$$

with a scatter $\Delta \log _{10}\left(c_{200}\right)=0.1$, where $h$ is the Hubble parameter [208]. In Figure 7 , the lines trace the mean of the circular velocity at $r=2 \mathrm{kpc}$ as a function of $V_{\max }$. Galaxies below the red band are those with less mass within $2 \mathrm{kpc}$ than expected from the predicted $\Lambda \mathrm{CDM}$ model. This is evidence for the presence of cores in such galaxies (see Figure 7). However, galaxies at large masses tend to have a higher circular velocity at $r=2 \mathrm{kpc}$ than expected from $\Lambda C D M$. This is explained by the non-negligible contribution of the baryons to the inner rotation curve in massive galaxies. We also note that the scatter in the circular velocity at $2 \mathrm{kpc}$ is reduced for galaxies below the red band as well as the mass increase (see Figure 7).

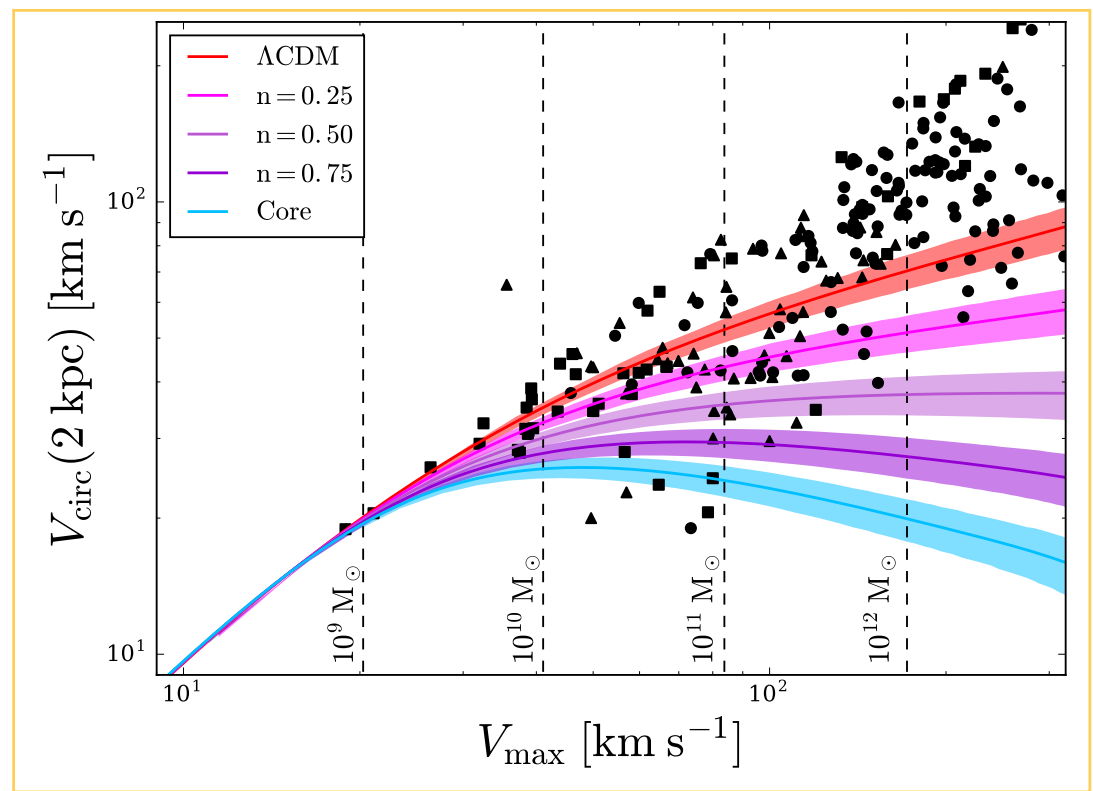

Figure 7. Diversity problem: Circular velocity at $r=2 \mathrm{kpc}$ versus the maximum circular velocity, $V_{\text {max }}$, for observed galaxies. The lines trace the mean $V_{\text {circ }}(2 \mathrm{kpc})$ as a function of $V_{\max }$ described by $\Lambda \mathrm{CDM}$ (red), coreNFW model (see Equation (12)) for $n=0.25$ (magenta), $n=0.5$ (purple), $n=0.75$ (violet) and $n=1$ (blue), where the width of the bands corresponds to the $1 \sigma$ scatter in DM halo concentrations (see Equation (13)). Observed galaxies with their observation type, such as HI (black square), $\mathrm{H} \alpha$ (black circle) and $\mathrm{HI}+\mathrm{H} \alpha$ (black triangle), were taken from the compilation by [77]. Galaxies below the red band are those with less mass within $2 \mathrm{kpc}$ than expected from the predicted $\Lambda \mathrm{CDM}$ model. 
Explaining this observed diversity demands a mechanism that creates cores of various sizes in only some galaxies, but not in others, over a wide range of $V_{\max }$. Nevertheless, these galaxies, formed in similar halos, have approximately the same baryonic mass and similar morphologies. Some diversity induced by differences in the distribution of the baryonic component was expected, but, clearly, the observed diversity is much greater than in simulations [31,54,209-211]. Further, we would expect that the DM is most affected in systems where baryons play a more important role, such as high-surface-brightness galaxies, whereas observations seem to suggest the opposite trend [70]. The observed diversity could be explained by the cusp regeneration phenomenon or by a different DM nature. Indeed, this behaviour of observed rotation curves is predicted by MOND theory [212].

\subsection{Inherent Cores}

The presence of the core appears to persist for dwarf galaxies that are DM-dominated and baryon-deficient. Thus, it is still unclear which dynamical process in a CDM environment can solve this puzzle. Another possibility is that the DM is more complex and hotter than simple CDM. A wide range of alternative DM models have been proposed over the last few decades. Mostly three main classes of alternative DM models have been simulated: warm dark matter (WDM) [143,213-217], self-interacting dark matter (SIDM) ( [218-223] and, for reviews, see [224,225]) and fuzzy dark matter (FDM). These fundamentally change the gravitational law [144,145,147,224,226-230]. Many of these alternative theories have been invoked to address $\Lambda$ CDM small-scale problems and, particularly, the cusp-core problem. FDM and SIDM, which are the two most recent alternative theories, are reviewed in this section. Moreover, primordial black holes have recently been proposed to explain the cusp-core problem as, from a dynamical perspective, they behave similarly to any other CDM candidate [231].

\subsubsection{Fuzzy Dark Matter}

As there is a current lack of evidence for any CDM particle, such as weakly interacting massive particles, DM, as an ultra-light scalar field with no self-interaction in the nonrelativistic limit, was introduced under the name of Fuzzy Dark Matter (FDM) [144,145]. This scalar field is assumed to be made up of very light particles with a mass of $\sim 10^{-22}-10^{-19} \mathrm{eV}$. One of the candidates for this alternative DM theory is the axion-like particles predicted by string theories [232]. Such a scalar field is then well-described in the non-relativistic limit by the coupled Schrödinger and Poisson equations [233]:

$$
\begin{gathered}
i \hbar \frac{\partial}{\partial t} \phi=-\frac{\hbar^{2}}{2 m} \nabla^{2} \phi+m U \psi, \\
\nabla^{2} U=4 \pi G \rho_{m},
\end{gathered}
$$

where $m$ is the mass of FDM particles. The mass density is defined as $\rho_{\mathrm{m}}=|\phi|^{2}$ and $U$ is the gravitational potential. Such ultra-light DM particles have a characteristic wavelength called the de Broglie wavelength:

$$
\lambda=1.19\left(\frac{10^{-22} \mathrm{eV}}{m}\right)\left(\frac{100 \mathrm{~km} \cdot \mathrm{s}^{-1}}{v}\right) \mathrm{kpc},
$$

where $v$ is the characteristic velocity. Equation (16) shows that the wavelength of a few $\mathrm{kpc}$ is of astrophysical size. Indeed, the small masses of ultra-light DM particles are associated with a very large de Broglie wavelength, where their quantum properties play an important role $[145,234,235]$. Thus, the de Broglie wavelength is of the order of the scales at which the cusp-core problem appears.

Axion-like particles are interesting DM candidates because they predict new structural and dynamical phenomena on scales of galaxies. When the de Broglie wavelength $\lambda$ is on the order of or larger than the inter-particle distance $d_{i}$, quantum effects will dominate. 
In fact, DM particles have huge occupancy numbers at these small scales. In the noninteracting Bose gas theory, the macroscopic occupation of the ground state is seen as condensation and this phenomenon is called Bose-Einstein condensation. In FDM, the particles form a Bose-Einstein condensate on galactic scales [145]. Figure 8 depicts that it results in a DM core at the halo's central region as the particles of the system are in the ground state, described by a single wave function $[230,235]$. Cosmological simulations of light DM found that the density profile of the innermost central region of the halos at redshift $z=0$ follows [230]:

$$
\rho(r)=\frac{\rho_{0}}{\left(1+0.091\left(r / r_{\mathrm{c}}\right)^{2}\right)^{8}} 10^{9} M_{\odot} \mathrm{kpc}^{-3},
$$

with

$$
\rho_{0}=0.019 m_{22}^{-2} r_{\mathrm{c}}^{-4} 10^{9} M_{\odot} \mathrm{kpc}^{-3},
$$

where $m_{22}=m / 10^{-22} \mathrm{eV}$ is the DM particle mass and $r_{\mathrm{c}}$ is the radius at which the density drops to one half its peak value for a halo at $z=0$. The central mass density of the core is given by [236]:

$$
M_{\mathrm{c}}=\frac{M_{\mathrm{h}}^{1 / 3}}{4}\left(4.4 \times 10^{7} m_{22}^{-3 / 2}\right)^{2 / 3},
$$

and

$$
r_{\mathrm{c}}=\frac{1.6}{m_{22}}\left(\frac{M_{\mathrm{h}}}{10^{9} M_{\odot}}\right)^{-1 / 3} \mathrm{kpc},
$$

where $M_{\mathrm{h}}$ is the halo mass. The heating mechanism is due to quantum fluctuations arising from the uncertainty principle. Indeed, the quantum pressure stabilises the gravitational collapse and prevents the formation of a cusp by suppressing the small-scale structures $[145,234,237]$. The condensate is a stable region where no clustering takes place (see Figure 8). These kpc cores offer one possible solution to the cusp-core problem.

However, when $\lambda \ll d_{\mathrm{i}}$, DM particles can be considered to be a classical system. Indeed, at large scales, condensation is broken and the system behaves as a system of individual massive particles [226]. Figure 8 shows that the outer region of FDM halo behaves similarly to CDM, which is well-approximated by the NFW [230]. Thus, the full density profile of halos can be written as:

$$
\rho(r)=\Theta\left(r_{t}-r\right) \rho_{c}+\Theta\left(r_{t}-r\right) \rho_{N F W},
$$

where the $\Theta$ is a step function and $r_{\mathrm{t}}$ is the transition radius, which marks the transition between the core profile and NFW profile. This specific scale is proportional to the core size as $r_{\mathrm{t}}=\alpha r_{\mathrm{c}}$ where $\alpha \sim 2-4$ [236].

FDM was introduced by the motivation to solve the core-cusp problem in DM halos of galaxies. As halo cores form naturally in FDM theory, this scenario is appealing in principle. However, some specific observations are necessary to verify this type of DM. The quantum nature of DM particles gives rise to specific density profiles and potential fluctuations that may affect delicate structures such as tidal streams and disks [238].

As illustrated above, any DM model that sets a universal core profile cannot fit observations. As such, baryonic physics must also play a significant role in shaping the DM profiles. Figure 9 illustrates the DM density profiles of a halo with a final virial mass of $10^{10}$ $\mathrm{M}_{\odot}$ at three different redshifts, assuming FDM, FDM with baryons and CDM with baryons. At the earliest redshift $z=5.6$, the CDM halo exhibits the highest central DM density with a cuspy profile, while the FDM halos show core profiles. The FDM halo with baryons has a lower density at the centre than the FDM-only halo because the baryon pressure delays its collapse. In contrast, at $z=4$, the FDM central density is more than one order of magnitude higher with baryons than without, exceeding the central DM density of the CDM halo. Indeed, in the presence of baryons, the cores grow by more than a factor of two. However, the core mass does not evolve over time if baryons are absent (see Figure 9). As DM cores 
become more massive and compact in the presence of baryons, observed rotation curves are likely harder to reconcile with FDM [239].
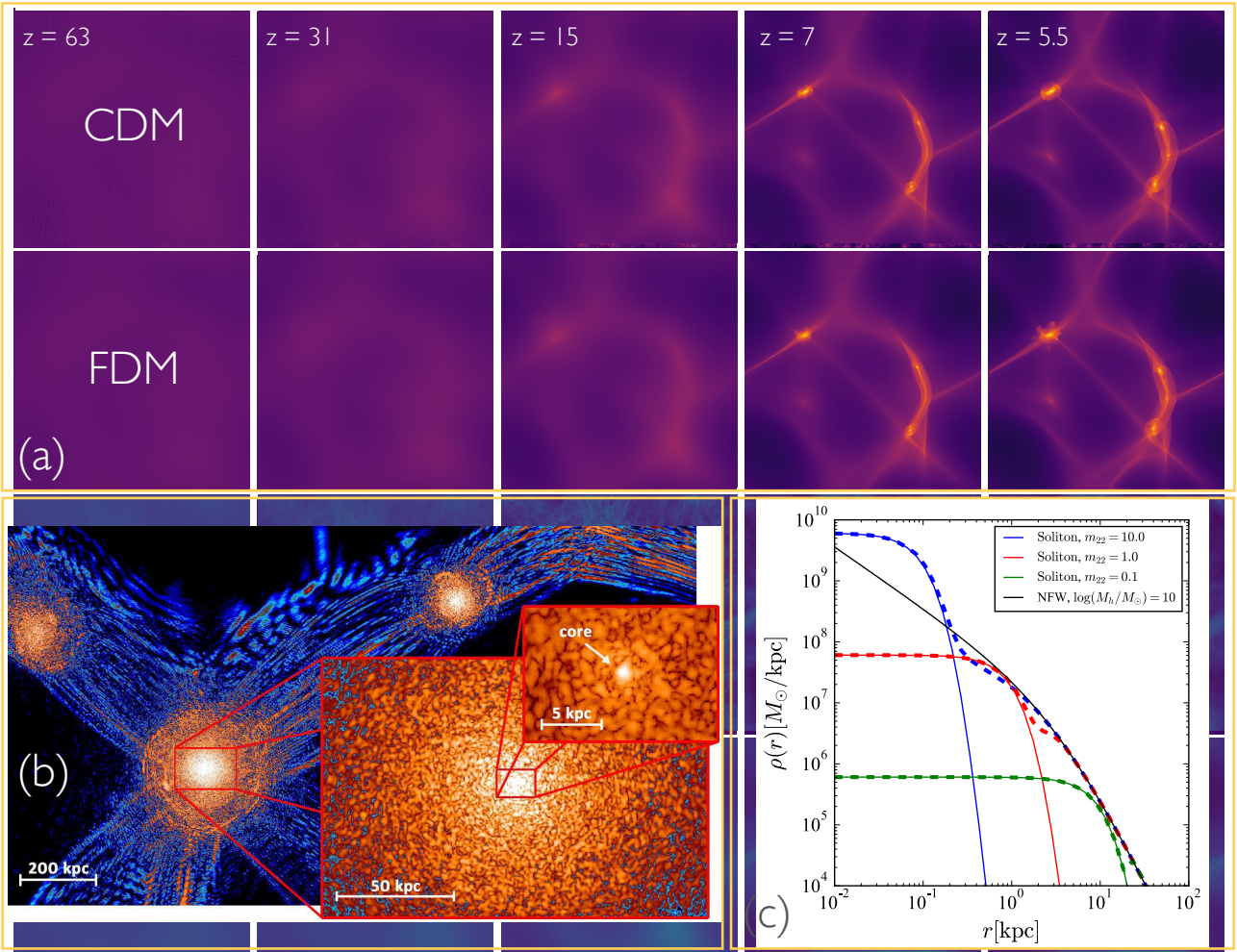

Figure 8. FDM properties: (a) Snapshots of the DM projected densities along the line of sight at $z=63,31,15,7$ and 5.5 under the CDM (upper panel) and FDM (lower panel) cosmologies. The two cosmological simulations have led to the formation of three $\sim 10^{9}-10^{10} \mathrm{M}_{\odot}$ halos. Snapshots highlight that FDM halos are connected via filaments, while CDM has filaments fragmented into subhalos. (b) Slice of density field of FDM simulation at different scales at $z=0.1$. We can distinguish the cores with a size of $\sim 0.3-1.6 \mathrm{kpc}$ in each halo. These DM cores grow as particles are accreted and surrounded by virialised halos. (c) DM profiles of a $10^{10} \mathrm{M}_{\odot}$ halo at $z=0$ for different values of $m_{22}$. Thin solid lines show the FDM core profiles for different axion masses. The thin black line shows the NFW profile of a $10^{10} \mathrm{M}_{\odot}$ halo at $z=0$. The thick dashed lines show the full halo profile that is a combination of the FDM profile transitioning to an NFW profile around $r=3 r_{\mathrm{c}}$. This figure is adapted from $[226,230,240]$.

Moreover, we expect that the DM distribution of centrally baryon-dominated galaxies, especially those containing supermassive black holes, is more strongly affected [241-244]. Figure 9 also shows the density profiles of FDM halos with masses from $10^{8} \mathrm{M}_{\odot}$ up to $10^{14} \mathrm{M}_{\odot}$, assuming an FDM particle mass of $10^{-22} \mathrm{eV}$. It can be seen that the black hole increases the central density only for $M_{\mathrm{h}} \geq 10^{13} \mathrm{M}_{\odot}$. This latter effect depends also on the FDM particle mass. Thus, black holes are most effective at modifying the DM distribution for higher halo masses and larger FDM particle masses. By numerically solving the Schrödinger-Poisson equations, it was shown that black holes decrease the core radius by increasing the central density of DM halos (see Figure 9). 

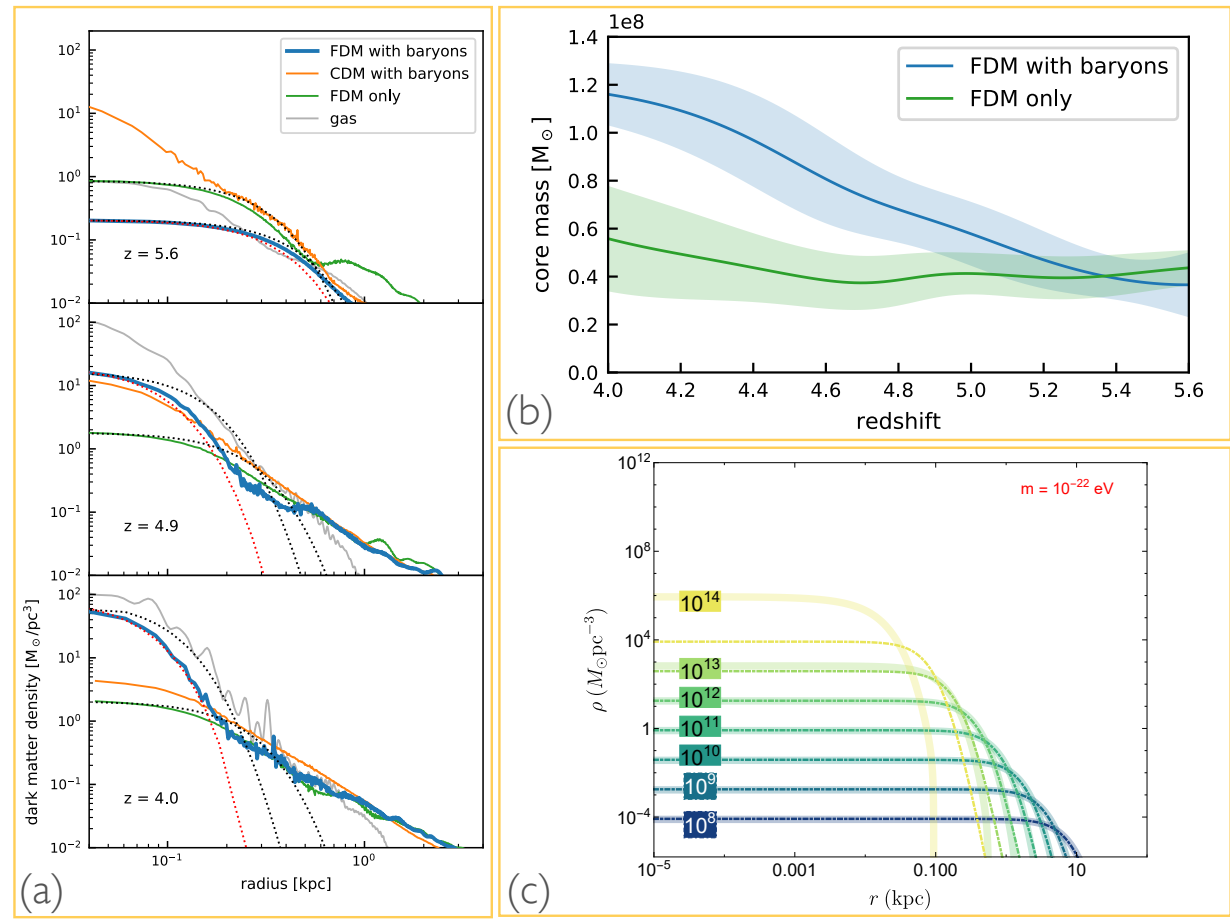

Figure 9. Adding baryons and black holes: (a) DM density profiles of a halo with a final virial mass of $10^{10} \mathrm{M}_{\odot}$ at three different redshifts, assuming FDM only, FDM with baryons and CDM with baryons. The FDM halo with baryons has a lower density at the centre than the FDM-only halo because the baryon pressure delays its collapse. (b) Evolution of the core mass over redshift for an FDM halo with (blue curve) and without baryons (green curve). The shaded regions represent the corresponding standard deviations. In the presence of baryons, the cores grow by more than a factor of two. (c) Density profiles of FDM halos with masses from $10^{8} \mathrm{M}_{\odot}$ up to $10^{14} \mathrm{M}_{\odot}$, assuming an FDM particle mass of $m=10^{-22} \mathrm{eV}$. The dot-dashed (thicker) lines correspond to DM halos without (with) a central black hole. It can be seen that the black hole increases the central density only for $M_{\mathrm{h}} \geq 10^{13} \mathrm{M}_{\odot}$. This figure is adapted from $[239,241]$.

Moreover, it not clear if FDM halos can be in line with the known galaxy scaling relation [236,240,242,245]. Figure 10 depicts the mass profiles of FDM halos with masses from $10^{9} \mathrm{M}_{\odot}$ up to $10^{11} \mathrm{M}_{\odot}$. For $m_{22}=0.1$, the predicted halo mass of the dwarf galaxies is too high given their dynamical state in the galaxy, and higher $m_{22}$ does not agree with the inferred slopes of Sculptor and Fornax. Low-mass axions $\left(m_{22}=0.1\right)$ can explain the observed mass profile slopes in Sculptor and Fornax [246-248]. However, at such low masses, the predicted halo masses of the ultra-faint dwarf galaxies such as Segue I are ruled out by dynamical friction arguments. In contrast, high-mass axions $\left(m_{22}=10\right)$ can explain the halo masses of the ultra-faint dwarf galaxies such as Draco II, Triangulum II and Segue I. For this axion mass, the predicted mass profiles do not agree with the observed slope of Fornax and Sculptor. The latter highlights the tensions concerning the FDM particle mass (see Figure 10). Indeed, stellar velocity measurements around the central BH of the MW constrained the FDM particle mass to $m<10^{-19} \mathrm{eV}$ [242]. However, the central motion of bulge stars in the MW favours a mass of $10^{-22} \mathrm{eV}$ [249]. A similar value was found by applying a Jeans analysis for MW dwarfs [250]. 


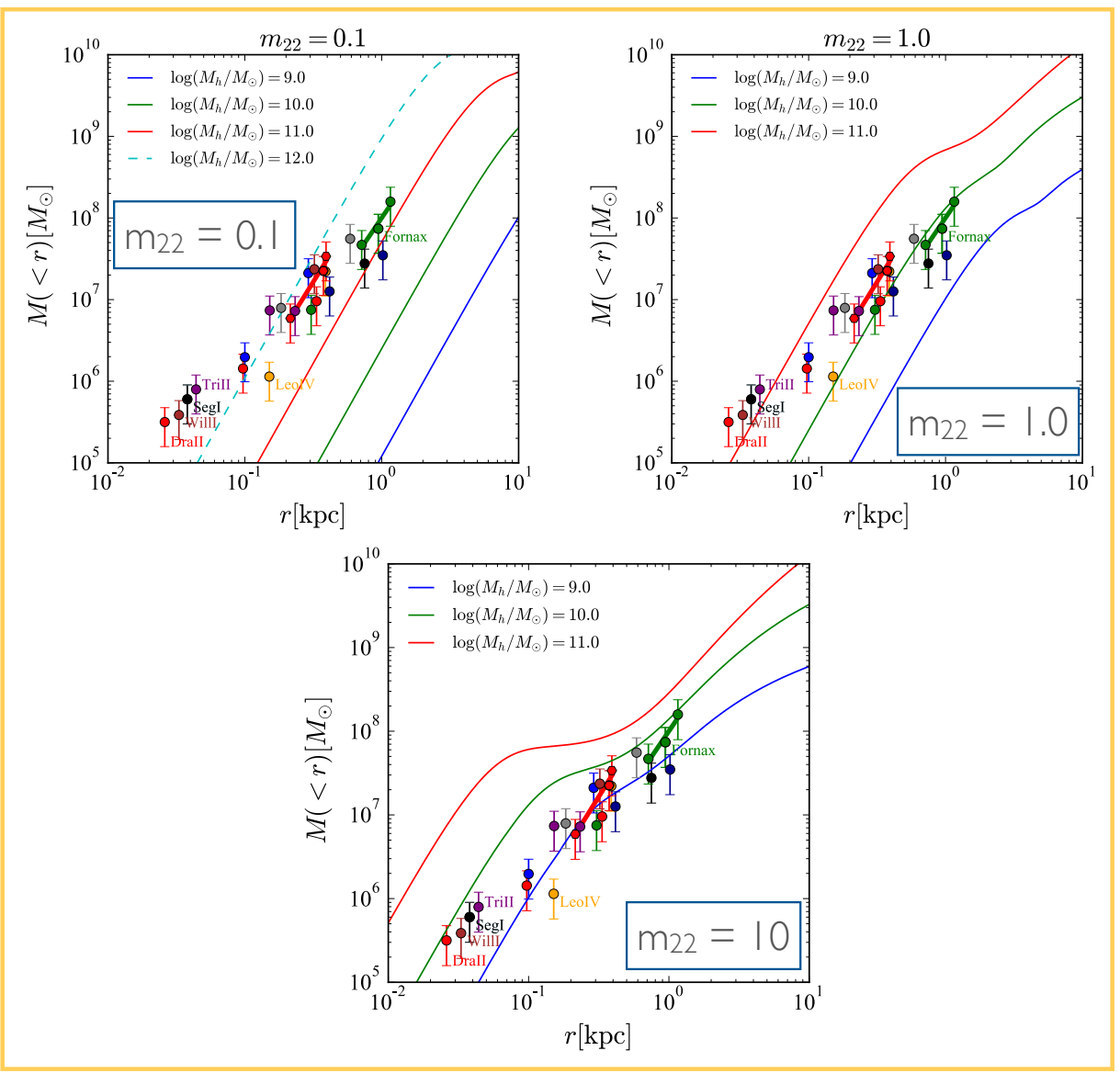

Figure 10. FDM inconsistencies: Mass profiles of FDM halos with masses from $10^{9} \mathrm{M}_{\odot}$ up to $10^{11} \mathrm{M}_{\odot}$. Left, middle and right panels represent the mass profiles corresponding to $m_{22}=0.1,1$ and 10, respectively. The individual data points for dwarf galaxies are collected from [129,251], and the slopes of Fornax and Sculptor (green and red lines) are from [102]. The profiles show a core region parametrised by Equation (19), which then follow an NFW profile at $r=3 r_{\mathrm{c}}$. These plots highlight the tensions concerning the FDM particle mass. This figure is adapted from [240].

Furthermore, it was pointed out that observational data indicate a positive scaling between the core radius $r_{\mathrm{c}}$ and the halo mass $M_{\mathrm{h}}$ [252,253]. In other words, we expect to have larger cores in massive galaxies. However, the FDM theory seems to predict the opposite behaviour. Indeed, the core radius is a decreasing function of the halo mass in an FDM universe, expressed as $r_{\mathrm{c}} \propto M^{\alpha}$ with $\alpha=1 / 3-5 / 9$ [230,254-259]. Thus, it seems very difficult for FDM to reproduce the observed relationship between core radius and halo mass in galaxies. To sum up, the FDM model provides a natural framework for the formation of DM cores but its predictions are in conflict with observations of galaxies.

\subsubsection{Self-Interacting Dark Matter}

In the $\Lambda \mathrm{CDM}$ model, DM is assumed to be collisionless. Another promising alternative is, therefore, self-interacting dark matter (SIDM) $[260,261]$, proposed to solve the smallscale problems and, more specifically, the cusp-core problem [223]. In this scenario, it was initially assumed that DM interactions are isotropic elastic scatterings with an interaction cross-section that is independent of velocity. Since the mass of the DM particle is not known, self-interactions are commonly quantified in terms of the cross-section per unit particle mass, $\sigma / m$, which is an important cosmological value for SIDM theories. The total number of interactions, $\Gamma$, that occurs per unit time is given by

$$
\Gamma \sim 0.1 \mathrm{Gyr}^{-1} \times\left(\frac{\rho_{\mathrm{dm}}}{0.1 M / \mathrm{pc}^{3}}\right)\left(\frac{\sigma / m}{1 \mathrm{~cm}^{2} \mathrm{~g}^{-1}}\right)\left(\frac{v_{\mathrm{rel}}}{50 \mathrm{kms}^{-1}}\right)
$$


where $m, \sigma$ and $v_{\text {rel }}$ are the DM particle mass, the cross-section and the relative velocity, respectively. The upper panel of Figure 11 compares the DM density distribution at large scales of CDM and SIDM halos. As the scattering rate $\Gamma$ is proportional to the DM density, SIDM halos have the same structure as CDM halos at large scales, where the DM interactions are negligible. Indeed, on the scale of their virial radius $\left(r_{\mathrm{vir}}=55 \mathrm{kpc}\right), \mathrm{CDM}$ and SIDM halos are nearly identical. Moreover, the collision rate is also negligible during the early Universe when DM structures form. Therefore, SIDM is consistent with observations of large-scale structures, predicted by $\Lambda \mathrm{CDM}[4,5]$. However, self-interactions perturb the inner density structure of a DM halo at late times. The upper panel of Figure 11 highlights that the SIDM halos at sub-galactic scales are less dense than in the CDM model due to the formation of cores.
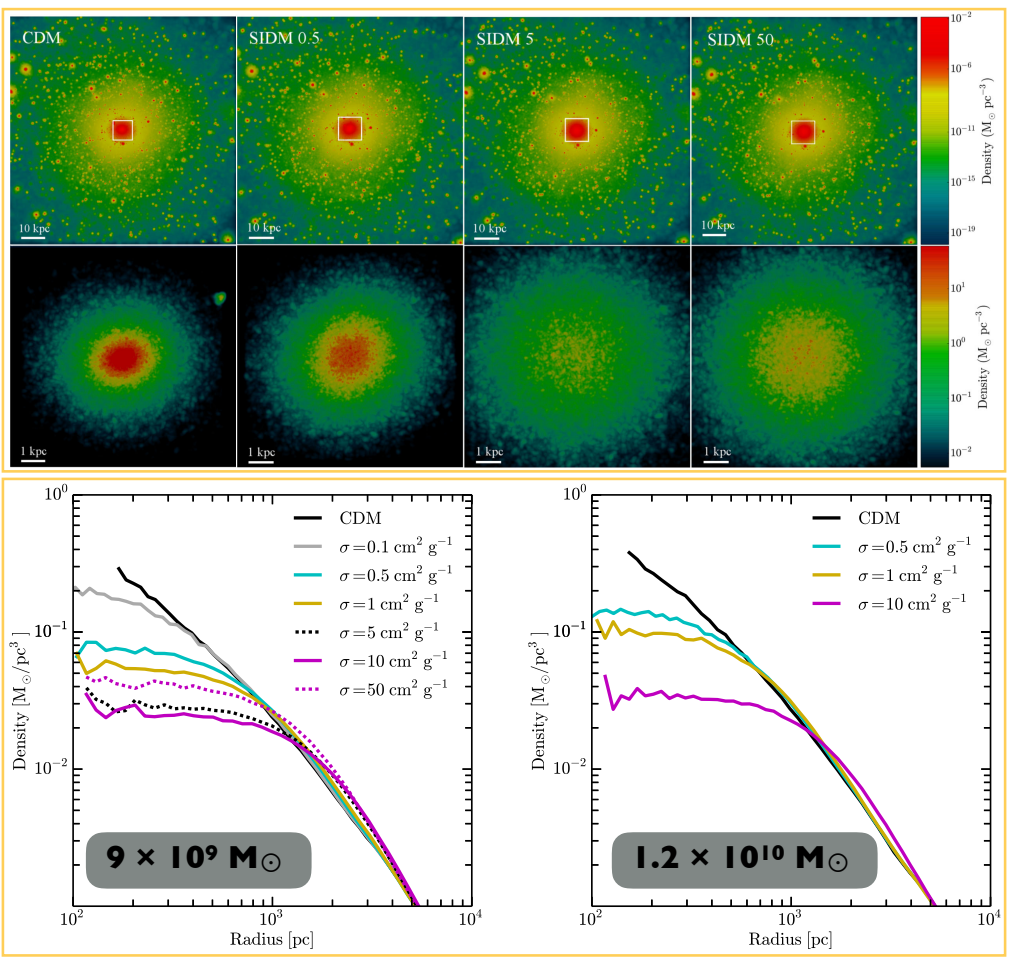

Figure 11. SIDM halo properties: Upper panel: DM density maps of CDM (left) and SIDM halo with a mass of $9 \times 10^{9} \mathrm{M}_{\odot}$ and $\sigma / m$ increasing from left to right in 100 and $10 \mathrm{kpc}$ boxes. SIDM halos have the same structure as CDM halos at large scales. At sub-galactic scales, the SIDM halos are less dense than in the CDM model due to the formation of cores. Lower panel: DM density profiles of $9 \times 10^{9}$ (left) and $1.2 \times 10^{10} \mathrm{M}_{\odot}$ (right) halos in CDM and SIDM models. SIDM runs have $\sigma / \mathrm{m}$ between 0.1 and $50 \mathrm{~cm}^{2} \mathrm{~g}^{-1}$. For $\sigma / m \geq 0.5 \mathrm{~cm}^{2} \mathrm{~g}^{-1}$, the self-interactions between DM particles produce central cores with a size depending on $\sigma / m$. This figure is adapted from [221].

A generic prediction for SIDM is that halos can form dense cores with size depending on the cross-section $\sigma / m[131,137,138,220,221,262-269]$, as shown in the lower panel of Figure 11. The redistribution of energy and momentum by DM particle collisions decreases the central density of DM halos, known as a cusp-to-core transition [222,223,263,269,270]. In other words, this heating transfer alters the inner region of halos by turning cuspy profiles into cored profiles. Core formations occur only if $\sigma / m$ is sufficiently large to ensure that the relatively high probability of scattering over time $T_{\text {age }}$ is comparable to the age of the halo: $\Gamma \times T_{\text {age }} \sim 1$. Figure 11 illustrates that the self-interactions between DM particles produce central cores for $\sigma / m \geq 0.5 \mathrm{~cm}^{2} \mathrm{~g}^{-1}$ in $9 \times 10^{9}-1.2 \times 10^{10} \mathrm{M}_{\odot}$ halos. Numerous simulations have then demonstrated that models with $\sigma / m \sim 0.5-10 \mathrm{~cm}^{2} / \mathrm{g}^{-1}$ produce DM cores in dwarf galaxies with sizes $\sim 0.3-1.5 \mathrm{kpc}[138,220,221,263-265]$, which could alleviate the cusp-core problem. In fact, the discrepancy with observations of low-surfacebrightness (LSB) galaxies having DM cores could be avoided in SIDM theory [223]. 
The viability of DM self-interacting as a cusp-core transformation mechanism depends on whether or not this cosmological model is consistent with all observations. In fact, it remains to be seen whether SIDM models are able to explain the observed cores from ultra-faint galaxies to galaxy clusters. The SIDM model requires compromises on the cross-section, which needs to be small enough to be observationally allowed but sufficiently large to alleviate the relevant small-scale problem. The first constraint on the SIDM crosssection was derived from galaxy clusters, which impose $\sigma / m<0.02 \mathrm{~cm}^{2} / \mathrm{g}$ [219]. Later, this constraint was revised and the inferred values of $\langle\sigma v\rangle / m$ for all six clusters are consistent with a constant cross-section $\sigma / m=0.1 \mathrm{~cm}^{2} \mathrm{~g}^{-1}$ according to the right panel of Figure 12 [264,271]. The left panel of Figure 12 shows that the SIDM model $\left(\sigma / m=0.1 \mathrm{~cm}^{2}\right.$ $\mathrm{g}^{-1}$ ) allowed by cluster constraints would be very similar to the CDM predictions. While the most massive CDM subhalos are inconsistent with the kinematics of the MW dSphs, the SIDM model can only alleviate this problem for $\sigma / m>1 \mathrm{~cm}^{2} \mathrm{~g}^{-1}$.
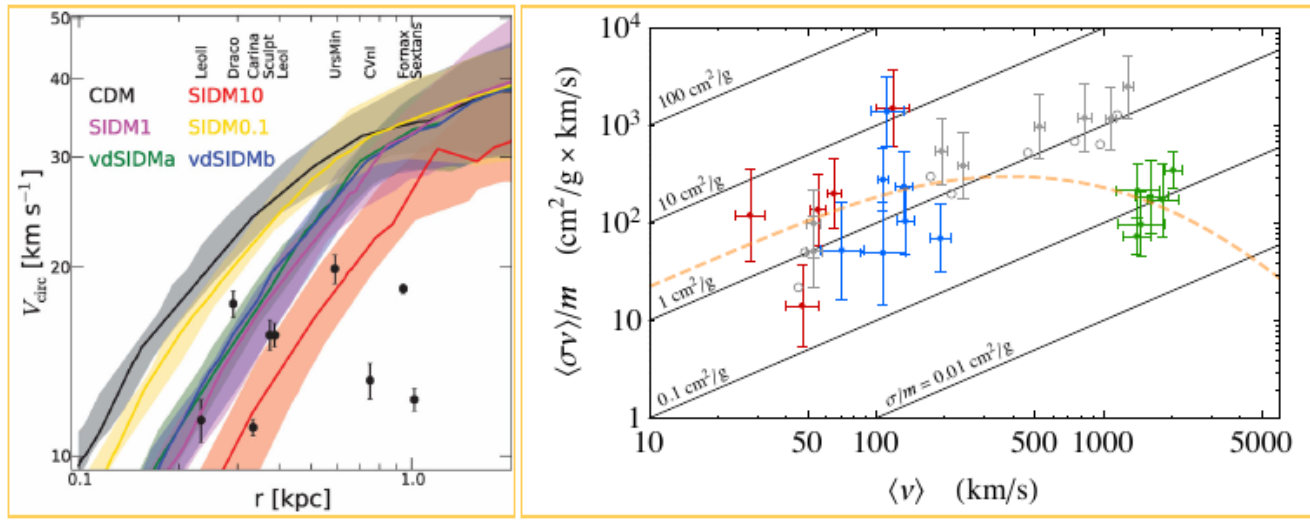

Figure 12. Constraints from observations: Left panel: Circular velocity profiles encompassing a distribution of 15 subhalos for CDM and SIDM models with a constant cross-section between 0.1 and $10 \mathrm{~cm}^{2} \mathrm{~g}^{-1}$. Black points with error bars correspond to the circular velocity within the half-light radii for nine MW dSphs [96,129]. While the most massive CDM subhalos are inconsistent with the kinematics of the MW dSphs, the SIDM model with $\sigma / m>1 \mathrm{~cm}^{2} \mathrm{~g}^{-1}$ can alleviate this problem. Right panel: Velocity-weighted cross-section per unit mass as a function of the mean collision velocity for dwarf galaxies (red), low-surface-brightness (LSB) galaxies (blue) and galaxy clusters (green). For comparison, SIDM $N$-body simulations with $\sigma / m=5-10 \mathrm{~cm}^{2} \mathrm{~g}^{-1}$ are represented by grey points. Diagonal lines show the corresponding cross-section $\sigma / m$. As $\sigma / m$ is not supposed to be constant in velocity, it is more convenient to invoke $\langle\sigma v\rangle / m$ rather than $\sigma / m$. The dashed curve represents the best fit for a velocity-dependent cross-section. This figure is adapted from [272].

If the self-scattering cross-section per unit mass is $\sim 1 \mathrm{~cm}^{2} \mathrm{~g}^{-1}$, SIDM models can solve the cusp-core problem at the scale of dwarf galaxies [220,263,265]. Figure 13 depicts the circular velocity profiles $V_{\text {circ }}$ of CDM and SIDM $\left(\sigma / m=1 \mathrm{~cm}^{2} \mathrm{~g}^{-1}\right)$ subhalos. Both CDM and SIDM subhalos match only 15-16 MW satellites. Nevertheless, SIDM theory with constant cross-section $\left(\sigma / m=1 \mathrm{~cm}^{2} \mathrm{~g}^{-1}\right)$ predicts DM subhalos with too low densities to match the observations of ultra-faint galaxies (see Figure 13). Thus, a constant cross-section of $\sigma / m=1 \mathrm{~cm}^{2} \mathrm{~g}^{-1}$ is likely to be inconsistent with the observed halo shapes of ultra-faint galaxies and several galaxy clusters. 


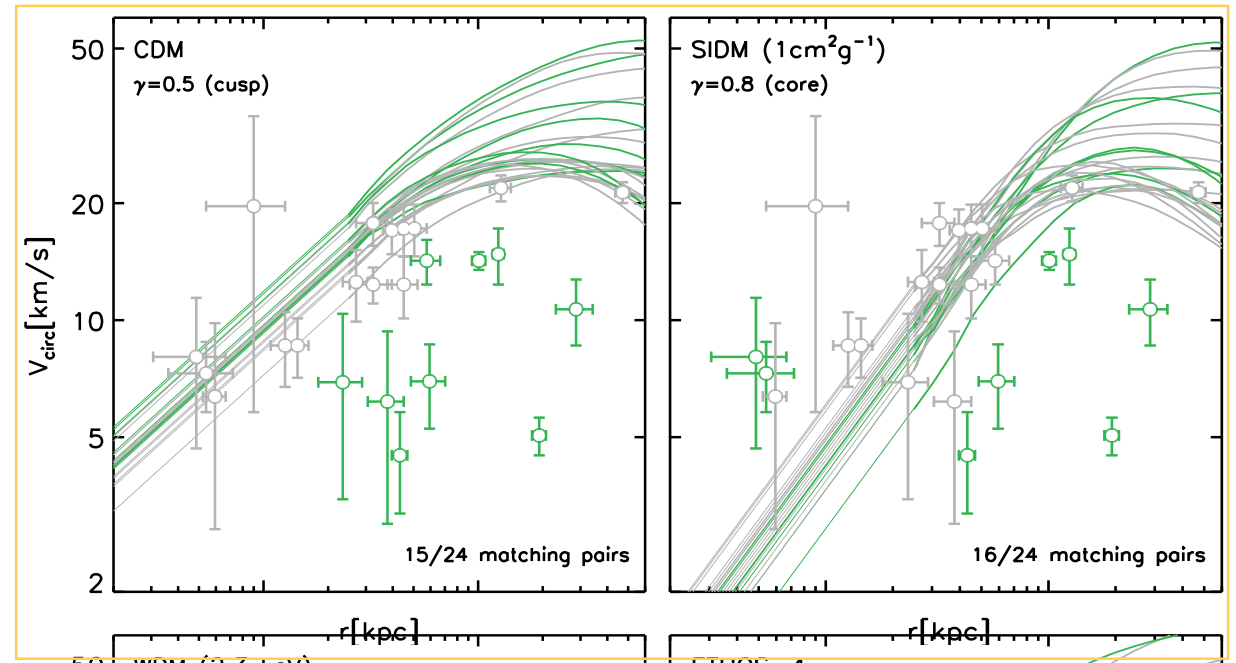

Figure 13. SIDM versus CDM: Circular velocity profiles $V_{\text {circ }}$ of CDM and SIDM $\left(\sigma / m=1 \mathrm{~cm}^{2} \mathrm{~g}^{-1}\right)$ subhalos within $300 \mathrm{kpc}$ from the centre of the simulated MW-like galaxies. Open symbols with error bars correspond to circular velocities at the half-light radius for 24 MW satellites [91,200]. Lines and symbols in grey (green) are consistent matches (mismatches) between simulated subhalos and data points. Both CDM and SIDM subhalos match only 15-16 MW satellites. This figure is adapted from [273].

Figure 14 highlights the possible velocity dependence discernible in these data from dwarfs to clusters. As $\sigma / m$ varies within a wide range, SIDM models, which assume a constant scattering cross-section, need to be abandoned since those that could solve the cusp-core problem in dwarfs seem to violate several astrophysical constraints. In order to alleviate the cusp-core problem and also match constraints at different scales, SIDM models need to have a velocity-dependent cross-section $\sigma(v)$ that decreases as the relative velocity of DM particles evolves from dwarfs to clusters, such as in Figure 14 [265,274,275]. For $\sigma / m>10 \mathrm{~cm}^{2} \mathrm{~g}^{-1}$, self-interactions between DM particles are frequent enough to entail a core collapse, which is a well-known mechanism in globular clusters [276], within a Hubble time in halos. Then, this results in the collapse of the core into a central cusp for SIDM halos [266,277-280]. As the vdSIDM model has cross-sections near and above the core collapse limit according to Figure 14, it produces a bimodal distribution composed of cusps and cores for MW-like subhalos. Indeed, the core collapse is responsible for this diversity, which is more consistent with cored brighter satellites and cuspy ultra-faint galaxies [273]. Thus, core collapses can be considered a mechanism to create a diverse population of dwarf-size halos, some of which would be cuspy and others that would have cores in velocity-dependent SIDM models [273,280-282].

All previous works are based on SIDM simulations, without taking into account baryonic physics. The inclusion of baryons into CDM simulations of dwarf galaxies has initially served to reduce the discrepancy between DM-only simulations and observations concerning the inner DM distribution. We have shown previously that baryonic feedback can reduce the central density of a cuspy DM halo. By including hydrodynamics in SIDM simulations, it was found that the DM inner regions of dwarf galaxies with stellar masses $M_{*}<10^{6}$ are nearly identical to the SIDM-only simulations $[131,137,283]$. Substantial DM cores are formed in both SIDM and SIDM + baryon simulations. It appears then that SIDM is more robust to feedback than CDM at dwarf scales [131,284]. This suggests that the faintest dwarf spheroidals provide excellent laboratories constraining SIDM models. Indeed, they are ideal targets as SIDM and CDM produce cores and cusps in these galaxies, respectively. 

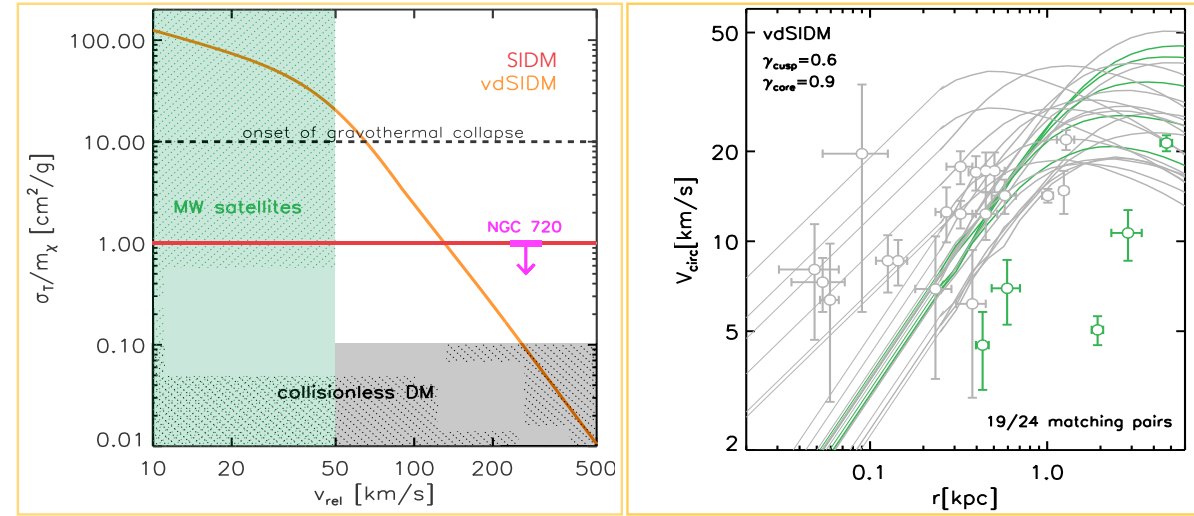

Figure 14. Velocity-dependent cross-section: Left panel: Cross-section as a function of the relative velocity. vdSIDM model consists of an SIDM with a strong velocity-dependent cross-section (orange line). The collisionless region is delimited by the black area $\sigma / m<0.1 \mathrm{~cm}^{2} \mathrm{~g}^{-1}$. For $\sigma / m>10 \mathrm{~cm}^{2} \mathrm{~g}^{-1}$, self-interactions between DM particles are frequent enough to result in core collapse within Hubble time in halos. The green area represents the relevant region for MW satellites. A constraint on the cross-section from the elliptical galaxy NGC720 is represented by a magenta arrow. Right panel: Circular velocity profiles $V_{\text {circ }}$ of vdSIDM (orange line in the left panel) subhalos within $300 \mathrm{kpc}$ from the centre of the simulated MW-like galaxies. Open symbols with error bars correspond to circular velocities at the half-light radius for $24 \mathrm{MW}$ satellites [91,200]. Lines and symbols in grey (green) are consistent matches (mismatches) between simulated subhalos and data points. As the vdSIDM model has cross-sections near and above the core collapse limit, it produces a bimodal distribution composed of cusps and cores for MW-like subhalos. This figure is adapted from [273].

For a high baryon concentration, it leads to a dense inner halo with a smaller core in the SIDM model [285]. Moreover, baryons can cause SIDM halos to core-collapse and become denser than DM halos in the presence of baryons [218,265,266,277,278]. As long as the baryonic component dominates the central region, core collapse can occur for $\sigma / m=0.5 \mathrm{~cm}^{2} \mathrm{~g}^{-1}$ [284]. This is the reason that the SIDM model predicts both cored and cuspy profiles, depending on the baryon concentration. As a result, the coupling between the SIDM and baryons also provides an explanation for the uniformity of the rotation curves [286-288].

Figure 15 shows that the logarithmic slope of the DM density profile, at $1.5 \%$ of the virial radius inferred from the SIDM fits, is correlated with the stellar mass [288]. Then, the SIDM + baryon model with an interaction cross-section of $3 \mathrm{~cm}^{2} \mathrm{~g}^{-1}$ can reproduce galaxy rotation curves from $\sim 50$ to $300 \mathrm{~km} \mathrm{~s}^{-1}[286,288,289]$. The slope $\alpha$ of SIDM fits, which include the baryonic impact, spans a large range from -0.5 to -2.5 , indicating that the SIDM model predicts both cored and cuspy halos. It was also pointed out that this reflects different baryon distributions in galaxies, which have a large impact on SIDM halos. Thus, the SIDM model predicts cored DM density profiles in low-surface-brightness galaxies and cuspy density profiles in high-surface-brightness galaxies. It therefore agrees best with observations. This coupling works because, within the characteristic scale of these galaxies, the DM and the baryonic masses are comparable. As halos, which host concentrated stellar populations, exhibit few differences in density profiles between CDM and SIDM models in the presence of baryons, the resulting DM core is effectively indistinguishable between CDM and SIDM (see Figure 15). It is possible that signatures in stellar kinematics could distinguish between these two core formation mechanisms, one impulsive (feedback) and the other adiabatic (SIDM) [139]. However, the impact of baryonic physics in ultra-faint galaxies is negligible, such that it is difficult to imagine how a population of dense ultrafaint galaxies can be accommodated with a constant cross-section of $\sigma / m=3 \mathrm{~cm}^{2} \mathrm{~g}^{-1}$ (see Figure 15) [273]. 


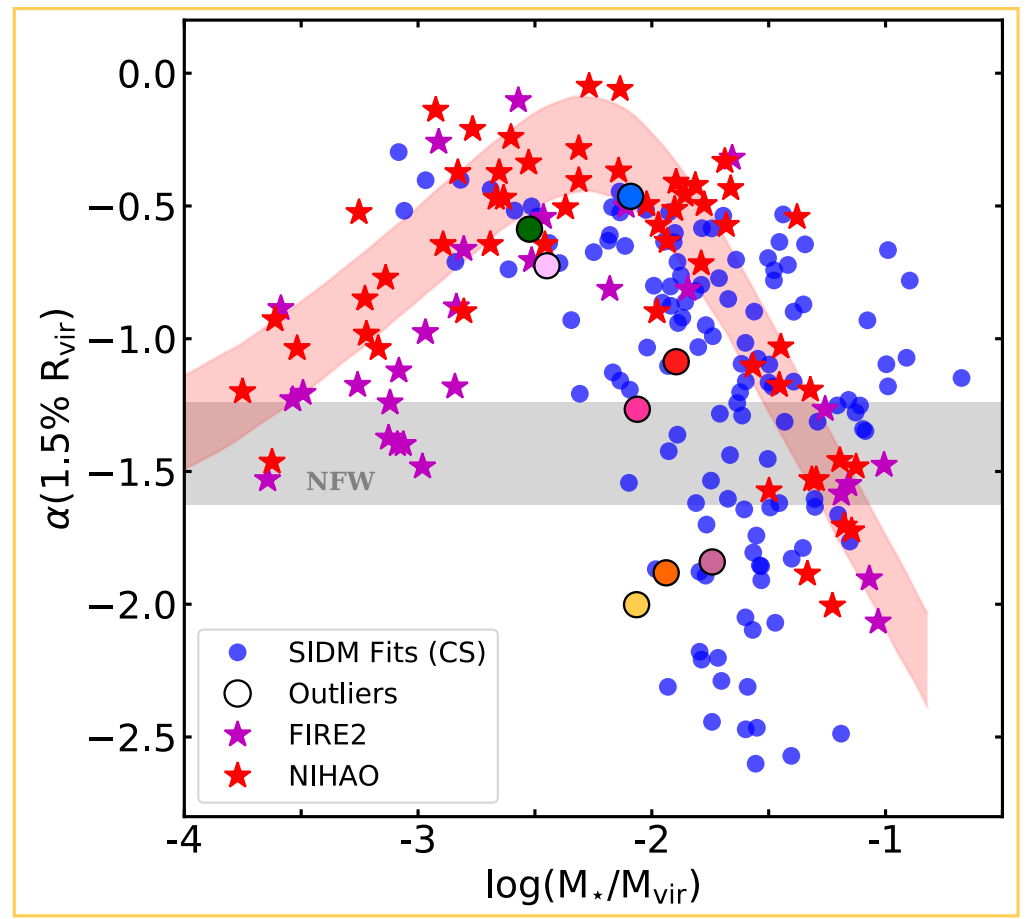

Figure 15. Baryon impact on SIDM halos: Inner DM density slope $\alpha$ at $r=0.015 r_{\text {vir }}$ as a function of $M_{*} / M_{\mathrm{vir}}$ at $z=0$ from SIDM fits [288], NIHAO [57] and FIRE-2 [58,290] hydrodynamical CDM simulations. The SIDM fits of the SPARC sample [291], which contains 135 galaxies, including the impact of baryons on the halo profile and compatible with a unique cross-section of $3 \mathrm{~cm}^{2} \mathrm{~g}^{-1}$. The shaded grey band shows the expected range of DM profile slopes for the NFW profile as derived from CDM-only simulations by including concentration scatter. The slope $\alpha$ of SIDM fits spans a large range from -0.5 to -2.5 , indicating that the SIDM model predicts both cored and cuspy halos. This figure is adapted from [289].

\subsubsection{Primordial Black Holes as Dark Matter Candidates}

Even if weakly interacting massive elementary particles are the most popular DM candidates, DM could be made up of macroscopic compact halo objects such as primordial black holes (PBHs) ([292-295]). These black holes could naturally be produced in the early Universe via cosmic inflation, without the need to appeal to new physics beyond the standard model $([296,297]$ and, for a recent review, [298]). One of the three allowed mass windows around $25-100 \mathrm{M}_{\odot}$ is of special interest in view of the recent detection of black-hole mergers by LIGO [299,300] and could potentially also detected by the Laser Interferometer Space Antenna (LISA) [301].

The cusp-core problem in $10^{7} \mathrm{M}_{\odot}$ halos such as low-mass dwarf galaxies by considering the possibility that a fraction of the DM is made up of PBHs was addressed by [231]. For a DM halo composed of CDM particles and $\mathrm{PBHs}(\mathrm{DM}=\mathrm{PBH}+\mathrm{CDM})$, the authors have defined the $\mathrm{PBH}+\mathrm{CDM}$ mass fraction as

$$
f_{\mathrm{m}}=\frac{M_{\mathrm{PBH}}}{M_{\mathrm{CDM}}}
$$

where $M_{\mathrm{PBH}}$ and $M_{\mathrm{CDM}}$ are the total masses of $\mathrm{PBHs}$ and $\mathrm{CDM}$ particles. It is known that, in collisionless systems such as globular clusters, massive stars fall towards the centre of the potential well and their energy is transferred to the lighter stars, which move away from the centre [302,303]. Consequently, the density profiles of lighter stars change due to this diffusion process [304-306]. In the same manner, Ref. [231] demonstrated using high-performance $\mathrm{N}$-body simulations on GPU that PBHs, as DM candidates, can induce a cusp-to-core transition in $\mathrm{PBH}+\mathrm{CDM}$ halos through gravitational heating from two principal mechanisms, dynamical friction by CDM particles on PBHs and two-body 
relaxation between $\mathrm{PBH}$ and CDM (see Figure 16). As the CDM particle velocity increases in the central region, the CDM density profile changes until core formation occurs. This figure demonstrates that core formation accompanies the dynamical heating of CDM particles.

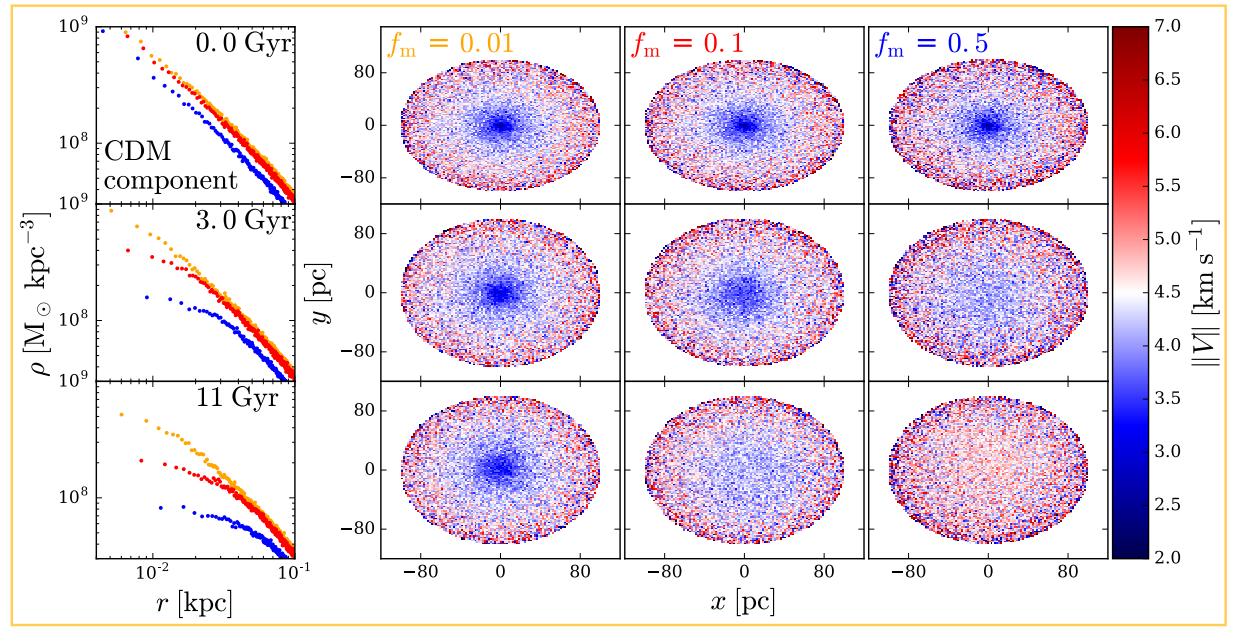

Figure 16. Cusp-to-core transition due to PBH-DM heating: Density profiles of the CDM component (left panels) and maps of the CDM mass-weighted velocity distribution projected face-on through a $100 \mathrm{pc}$ (right panels) over time. The $\mathrm{PBH}+\mathrm{CDM}$ halos are populated with $100 \mathrm{M}_{\odot} \mathrm{PBHs}$, assuming $r_{\mathrm{s}}^{\mathrm{PBH}}=r_{\mathrm{s}}^{\mathrm{CDM}}$ and different $f_{\mathrm{m}}=0.5$. Core formation accompanies the dynamical heating of CDM particles. This figure is reprinted from [289].

This suggests that this core formation mechanism works with a lower limit on the $\mathrm{PBH}$ mass fraction of $1 \%$ of the total dwarf galaxy dark matter content [231]. This cusp-to-core transition takes between 1 and 8 Gyr to appear, depending on the fraction $f_{m}$, the $\mathrm{PBH}$ mass $m_{\mathrm{PBH}}$ and the $\mathrm{PBH}$ scale radius $r_{\mathrm{s}}^{\mathrm{PBH}}$ [231]. As cores occur naturally in $\mathrm{PBH}+\mathrm{CDM}$ halos without the presence of baryons, there is no cusp-core problem in this alternative theory. However, this mechanism seems only efficient in low-mass galaxies as the core formation time is proportional to the halo mass. This is the reason that cores in higher-mass galaxies could form only via a hierarchical scenario-in other words, through halo mergers. Even if this alternative theory was already investigated in a cosmological context with only $\mathrm{DM}+\mathrm{PBHs}$ [307], this mechanism needs to be tested in the presence of baryons.

\section{Conclusions}

As understanding how dark matter (DM) is distributed in the central region of a galaxy is directly related to one of the major unsolved problems in astrophysics - the nature of $\mathrm{DM}$ - it is not surprising that the cusp-core problem in dwarf galaxies in accordance with observations still remains a challenge. This review was intended to discuss all the main research avenues for solutions to this small-scale issue within cold dark matter (CDM) but also in alternative theories.

In the future, the cusp-core problem must be approached from two main angles. First, an accurate inference of the DM density profile from observations is necessary. This should become possible with more radial velocities in the central regions of dwarfs thanks to future Gaia data release [308]. Currently, DM densities are barely constrained observationally at $\sim 100$ pc scales. As stressed by [126], we need more member stars for dwarfs to properly use the Jeans analysis by assuming realistic non-spherical geometry for halos. Then, we could focus in greater depth on other dwarfs than Fornax, which has been extensively investigated because of its large stellar mass (see Table 1).

Second, all core formation mechanisms within CDM need to be addressed in a cosmological context to check their efficiency during the Universe's formation, but it is also crucial to find observational signatures of these mechanisms in order to distinguish and maybe exclude some of them. For instance, if tidal effects are responsible for DM core formation in 
our local dwarfs, such tidal tails should be detectable in future surveys [202]. Concerning alternative DM theories, efforts must be pursued in constraining their additional degrees of freedom compared to CDM. Very recently, a new upper limit on the self-interacting scattering cross-section in the SIDM universe was imposed based on comparing the measurements of the central density at 150 pc of subhalos in a high-resolution cosmological simulation and our local dwarf galaxies [309].

As outlined in the review, the contribution of baryons in a gravitational and hydrodynamical fashion to the distribution of DM within galaxies is non-negligible. However, the presence of baryons can potentially bias our understanding of the DM's properties. For instance, both stellar feedback and SIDM can initiate cusp-to-core transformation in dwarf halos. However, it was pointed out these core mechanisms act on different timescales at which they affect the gravitational potential [273]. Furthermore, it was demonstrated that they could have a distinct signature in the velocity dispersion profiles of stars [310]. Such observable properties can be used to distinguish these two mechanisms.

Future missions such as the James Webb Space Telescope have the ambition to give us a direct insight into DM halos of very-high-redshift galaxies. These very old DM structures will not have been altered by the effects of the environment yet. The presence of DM cores in these galaxies will dramatically favour alternative theories where cores emerge naturally. On the contrary, the absence of cores will reinforce the CDM model and dynamical perturbers such as stellar feedback of infalling structures will be responsible for the formation of cores at low redshift.

Funding: This research received no external funding.

Institutional Review Board Statement: Not applicable.

Informed Consent Statement: Not applicable.

Data Availability Statement: Not applicable.

Acknowledgments: I thank the three reviewers for their constructive feedback, which helped to improve the quality of the manuscript. I thank Joseph Silk for his useful comments and suggestions. I also thank Eduardo Vitral for the illuminating discussions about Gaia data.

Conflicts of Interest: The author declares no conflict of interest.

\section{References}

1. Blumenthal, G.R.; Faber, S.M.; Primack, J.R.; Rees, M.J. Formation of galaxies and large-scale structure with cold dark matter. Nature 1984, 311, 517-525. doi: 10.1038/311517a0. [CrossRef]

2. Spergel, D.N.; Verde, L.; Peiris, H.V.; Komatsu, E.; Nolta, M.R.; Bennett, C.L.; Halpern, M.; Hinshaw, G.; Jarosik, N.; Kogut, A.; et al. First-Year Wilkinson Microwave Anisotropy Probe (WMAP) Observations: Determination of Cosmological Parameters. Astrophys. J. Suppl. Ser. 2003, 148, 175-194. doi: 10.1086/377226. [CrossRef]

3. Croft, R.A.C.; Weinberg, D.H.; Bolte, M.; Burles, S.; Hernquist, L.; Katz, N.; Kirkman, D.; Tytler, D. Toward a Precise Measurement of Matter Clustering: Ly $\alpha$ Forest Data at Redshifts 2-4. Astrophys. J. 2002, 581, 20-52. doi: 10.1086/344099. [CrossRef]

4. Springel, V.; Frenk, C.S.; White, S.D.M. The large-scale structure of the Universe. Nature 2006, 440, 1137-1144. doi: 10.1038/nature04805. [CrossRef]

5. Trujillo-Gomez, S.; Klypin, A.; Primack, J.; Romanowsky, A.J. Galaxies in $\Lambda$ CDM with Halo Abundance Matching: LuminosityVelocity Relation, Baryonic Mass-Velocity Relation, Velocity Function, and Clustering. Astrophys. J. 2011, 742, $16 . \quad$ doi: 10.1088/0004-637X/742/1/16. [CrossRef]

6. Frenk, C.S.; White, S.D.M.; Davis, M.; Efstathiou, G. The Formation of Dark Halos in a Universe Dominated by Cold Dark Matter. Astrophys. J. 1988, 327, 507. doi: 10.1086/166213. [CrossRef]

7. Dubinski, J.; Carlberg, R.G. The Structure of Cold Dark Matter Halos. Astrophys. J. 1991, 378, 496. doi: 10.1086/170451. [CrossRef]

8. Gelb, J.M.; Bertschinger, E. Cold Dark Matter. I. The Formation of Dark Halos. Astrophys. J. 1994, 436, 467. doi: 10.1086/174922. [CrossRef]

9. Navarro, J.F.; Frenk, C.S.; White, S.D.M. The Structure of Cold Dark Matter Halos. Astrophys. J. 1996, 462, 563. doi: 10.1086/177173. [CrossRef]

10. Navarro, J.F.; Frenk, C.S.; White, S.D.M. A Universal Density Profile from Hierarchical Clustering. Astrophys. J. 1997, 490, 493-508. doi: 10.1086/304888. [CrossRef]

11. Fukushige, T.; Makino, J. On the Origin of Cusps in Dark Matter Halos. Astrophys. J. Lett. 1997, 477, L9-L12. doi: 10.1086/310516. [CrossRef] 
12. Moore, B.; Governato, F.; Quinn, T.; Stadel, J.; Lake, G. Resolving the Structure of Cold Dark Matter Halos. Astrophys. J. Lett. 1998, 499, L5-L8. doi: 10.1086/311333. [CrossRef]

13. Navarro, J.F.; Ludlow, A.; Springel, V.; Wang, J.; Vogelsberger, M.; White, S.D.M.; Jenkins, A.; Frenk, C.S.; Helmi, A. The diversity and similarity of simulated cold dark matter haloes. Mon. Not. R. Astron. Soc. 2010, 402, 21-34. doi: 10.1111/j.13652966.2009.15878.x. [CrossRef]

14. Einasto, J. On the Construction of a Composite Model for the Galaxy and on the Determination of the System of Galactic Parameters. Tr. Astrofiz. Inst. Alma-Ata 1965, 5, 87-100.

15. Graziani, L.; de Bennassuti, M.; Schneider, R.; Kawata, D.; Salvadori, S. The history of the dark and luminous side of Milky Way-like progenitors. Mon. Not. R. Astron. Soc. 2017, 469, 1101-1116. doi: 10.1093/mnras/stx900. [CrossRef]

16. Mateo, M. Strange Dark Matters in Nearby Dwarf Galaxies. Magellanic Clouds and Other Dwarf Galaxies. 1998. pp. 53-66. Available online: https:/ / ui.adsabs.harvard.edu/abs/1998mcdg.proc...53M/abstract (accessed on 27 October 2021).

17. McConnachie, A.W. The Observed Properties of Dwarf Galaxies in and around the Local Group. Astron. J. 2012, 144, 4. doi: 10.1088/0004-6256/144/1/4. [CrossRef]

18. Moore, B. Evidence against dissipation-less dark matter from observations of galaxy haloes. Nature 1994, 370, 629-631. doi: 10.1038/370629a0. [CrossRef]

19. Flores, R.A.; Primack, J.R. Observational and Theoretical Constraints on Singular Dark Matter Halos. Astrophys. J. Lett. 1994, 427, L1. doi: 10.1086/187350. [CrossRef]

20. Burkert, A. The Structure of Dark Matter Halos in Dwarf Galaxies. Astrophys. J. Lett. 1995, 447, L25-L28. doi: 10.1086/309560. [CrossRef]

21. Marchesini, D.; D'Onghia, E.; Chincarini, G.; Firmani, C.; Conconi, P.; Molinari, E.; Zacchei, A. H $\alpha$ Rotation Curves: The Soft Core Question. Astrophys. J. 2002, 575, 801-813. doi: 10.1086/341475. [CrossRef]

22. de Blok, W.J.G.; Bosma, A.; McGaugh, S. Simulating observations of dark matter dominated galaxies: Towards the optimal halo profile. Mon. Not. R. Astron. Soc. 2003, 340, 657-678. doi: 10.1046/j.1365-8711.2003.06330.x. [CrossRef]

23. Rhee, G.; Valenzuela, O.; Klypin, A.; Holtzman, J.; Moorthy, B. The Rotation Curves of Dwarf Galaxies: A Problem for Cold Dark Matter? Astrophys. J. 2004, 617, 1059-1076. doi: 10.1086/425565. [CrossRef]

24. van den Bosch, F.C.; Swaters, R.A. Dwarf galaxy rotation curves and the core problem of dark matter haloes. Mon. Not. R. Astron. Soc. 2001, 325, 1017-1038. doi: 10.1046/j.1365-8711.2001.04456.x. [CrossRef]

25. Spekkens, K.; Giovanelli, R.; Haynes, M.P. The Cusp/Core Problem in Galactic Halos: Long-Slit Spectra for a Large Dwarf Galaxy Sample. Astron. J. 2005, 129, 2119-2137. doi: 10.1086/429592. [CrossRef]

26. Walter, F.; Brinks, E.; de Blok, W.J.G.; Bigiel, F.; Kennicutt, R.C., Jr.; Thornley, M.D.; Leroy, A. THINGS: The H I Nearby Galaxy Survey. Astron. J. 2008, 136, 2563-2647. doi: 10.1088/0004-6256/136/6/2563. [CrossRef]

27. Hunter, D.A.; Ficut-Vicas, D.; Ashley, T.; Brinks, E.; Cigan, P.; Elmegreen, B.G.; Heesen, V.; Herrmann, K.A.; Johnson, M.; Oh, S.H.; et al. Little Things. Astron. J. 2012, 144, 134. doi: 10.1088/0004-6256/144/5/134. [CrossRef]

28. Trachternach, C.; de Blok, W.J.G.; Walter, F.; Brinks, E.; Kennicutt, R.C., Jr. Dynamical Centers and Noncircular Motions in THINGS Galaxies: Implications for Dark Matter Halos. Astron. J. 2008, 136, 2720-2760. doi: 10.1088/0004-6256/136/6/2720. [CrossRef]

29. Oh, S.H.; de Blok, W.J.G.; Walter, F.; Brinks, E.; Kennicutt, R.C., Jr. High-Resolution Dark Matter Density Profiles of THINGS Dwarf Galaxies: Correcting for Noncircular Motions. Astron. J. 2008, 136, 2761-2781. doi: 10.1088/0004-6256/136/6/2761. [CrossRef]

30. de Blok, W.J.G.; Walter, F.; Brinks, E.; Trachternach, C.; Oh, S.H.; Kennicutt, R.C., Jr. High-Resolution Rotation Curves and Galaxy Mass Models from THINGS. Astron. J. 2008, 136, 2648-2719. doi: 10.1088/0004-6256/136/6/2648. [CrossRef]

31. Oh, S.H.; de Blok, W.J.G.; Brinks, E.; Walter, F.; Kennicutt, R.C., Jr. Dark and Luminous Matter in THINGS Dwarf Galaxies. Astron. J. 2011, 141, 193. doi: 10.1088/0004-6256/141/6/193. [CrossRef]

32. Oh, S.H.; Hunter, D.A.; Brinks, E.; Elmegreen, B.G.; Schruba, A.; Walter, F.; Rupen, M.P.; Young, L.M.; Simpson, C.E.; Johnson, M.C.; et al. High-resolution Mass Models of Dwarf Galaxies from LITTLE THINGS. Astron. J. 2015, 149, 180. doi: 10.1088/00046256/149/6/180. [CrossRef]

33. Navarro, J.F.; Eke, V.R.; Frenk, C.S. The cores of dwarf galaxy haloes. Mon. Not. R. Astron. Soc. 1996, 283, L72-L78. doi: 10.1093/mnras/283.3.L72. [CrossRef]

34. Ciardi, B.; Ferrara, A. The First Cosmic Structures and Their Effects. Space Sci. Rev. 2005, 116, 625-705. doi: 10.1007/s11214-005-3592-0. [CrossRef]

35. White, S.D.M.; Frenk, C.S. Galaxy Formation through Hierarchical Clustering. Astrophys. J. 1991, 379, 52. doi: 10.1086/170483. [CrossRef]

36. White, S.D.M.; Rees, M.J. Core condensation in heavy halos: a two-stage theory for galaxy formation and clustering. Mon. Not. R. Astron. Soc. 1978, 183, 341-358. doi: 10.1093/mnras/183.3.341. [CrossRef]

37. Larson, R.B. Effects of supernovae on the early evolution of galaxies. Mon. Not. R. Astron. Soc. 1974, 169, 229-246. doi: 10.1093/mnras/169.2.229. [CrossRef]

38. Dekel, A.; Silk, J. The Origin of Dwarf Galaxies, Cold Dark Matter, and Biased Galaxy Formation. Astrophys. J. 1986, 303, 39. doi: 10.1086/164050. [CrossRef]

39. Blumenthal, G.R.; Faber, S.M.; Flores, R.; Primack, J.R. Contraction of Dark Matter Galactic Halos Due to Baryonic Infall. Astrophys. J. 1986, 301, 27. doi: 10.1086/163867. [CrossRef]

40. Gnedin, O.Y.; Kravtsov, A.V.; Klypin, A.A.; Nagai, D. Response of Dark Matter Halos to Condensation of Baryons: Cosmological Simulations and Improved Adiabatic Contraction Model. Astrophys. J. 2004, 616, 16-26. doi: 10.1086/424914. [CrossRef] 
41. Abadi, M.G.; Navarro, J.F.; Fardal, M.; Babul, A.; Steinmetz, M. Galaxy-induced transformation of dark matter haloes. Mon. Not. R. Astron. Soc. 2010, 407, 435-446. doi: 10.1111/j.1365-2966.2010.16912.x. [CrossRef]

42. Schaller, M.; Robertson, A.; Massey, R.; Bower, R.G.; Eke, V.R. The offsets between galaxies and their dark matter in $\Lambda$ cold dark matter. Mon. Not. R. Astron. Soc. 2015, 453, L58-L62. doi: 10.1093/mnrasl/slv104. [CrossRef]

43. Gnedin, O.Y.; Zhao, H. Maximum feedback and dark matter profiles of dwarf galaxies. Mon. Not. R. Astron. Soc. 2002, 333, 299-306. doi: 10.1046/j.1365-8711.2002.05361.x. [CrossRef]

44. Read, J.I.; Gilmore, G. Mass loss from dwarf spheroidal galaxies: the origins of shallow dark matter cores and exponential surface brightness profiles. Mon. Not. R. Astron. Soc. 2005, 356, 107-124. doi: 10.1111/j.1365-2966.2004.08424.x. [CrossRef]

45. Brooks, A.M.; Zolotov, A. Why Baryons Matter: The Kinematics of Dwarf Spheroidal Satellites. Astrophys. J. 2014, 786, 87. doi: 10.1088/0004-637X/786/2/87. [CrossRef]

46. Mashchenko, S.; Wadsley, J.; Couchman, H.M.P. Stellar Feedback in Dwarf Galaxy Formation. Science 2008, 319, 174. doi: 10.1126/science.1148666. [CrossRef] [PubMed]

47. Macciò, A.V.; Stinson, G.; Brook, C.B.; Wadsley, J.; Couchman, H.M.P.; Shen, S.; Gibson, B.K.; Quinn, T. Halo Expansion in Cosmological Hydro Simulations: Toward a Baryonic Solution of the Cusp/Core Problem in Massive Spirals. Astrophys. J. Lett. 2012, 744, L9. doi: 10.1088/2041-8205/744/1/L9. [CrossRef]

48. Pontzen, A.; Governato, F. How supernova feedback turns dark matter cusps into cores. Mon. Not. R. Astron. Soc. 2012, 421, 3464-3471. doi: 10.1111/j.1365-2966.2012.20571.x. [CrossRef]

49. Pontzen, A.; Governato, F. Cold dark matter heats up. Nature 2014, 506, 171-178. doi: 10.1038/nature12953. [CrossRef]

50. Madau, P.; Dickinson, M. Cosmic Star-Formation History. Annu. Rev. Astron. Astrophys. 2014, 52, 415-486. annurev-astro-081811-125615. [CrossRef]

51. Freundlich, J.; Dekel, A.; Jiang, F.; Ishai, G.; Cornuault, N.; Lapiner, S.; Dutton, A.A.; Macciò, A.V. A model for core formation in dark matter haloes and ultra-diffuse galaxies by outflow episodes. Mon. Not. R. Astron. Soc. 2020, 491, 4523-4542. doi: 10.1093/mnras/stz3306. [CrossRef]

52. Martizzi, D.; Teyssier, R.; Moore, B. Cusp-core transformations induced by AGN feedback in the progenitors of cluster galaxies Mon. Not. R. Astron. Soc. 2013, 432, 1947-1954. doi: 10.1093/mnras/stt297. [CrossRef]

53. Silk, J. Feedback by Massive Black Holes in Gas-rich Dwarf Galaxies. Astrophys. J. Lett. 2017, 839, L13. doi: 10.3847/20418213/aa67da. [CrossRef]

54. Governato, F.; Brook, C.; Mayer, L.; Brooks, A.; Rhee, G.; Wadsley, J.; Jonsson, P.; Willman, B.; Stinson, G.; Quinn, T.; et al. Bulgeless dwarf galaxies and dark matter cores from supernova-driven outflows. Nature 2010, 463, 203-206. doi: 10.1038/nature08640. [CrossRef] [PubMed]

55. Zolotov, A.; Brooks, A.M.; Willman, B.; Governato, F.; Pontzen, A.; Christensen, C.; Dekel, A.; Quinn, T.; Shen, S.; Wadsley, J. Baryons Matter: Why Luminous Satellite Galaxies have Reduced Central Masses. Astrophys. J. 2012, 761, 71. doi: 10.1088/0004637X/761/1/71. [CrossRef]

56. Di Cintio, A.; Brook, C.B.; Dutton, A.A.; Macciò, A.V.; Stinson, G.S.; Knebe, A. A mass-dependent density profile for dark matter haloes including the influence of galaxy formation. Mon. Not. R. Astron. Soc. 2014, 441, 2986-2995. doi: 10.1093/mnras/stu729. [CrossRef]

57. Tollet, E.; Macciò, A.V.; Dutton, A.A.; Stinson, G.S.; Wang, L.; Penzo, C.; Gutcke, T.A.; Buck, T.; Kang, X.; Brook, C.; et al. NIHAO-IV: core creation and destruction in dark matter density profiles across cosmic time. Mon. Not. R. Astron. Soc. 2016, 456, 3542-3552. doi: 10.1093/mnras/stv2856. [CrossRef]

58. Hopkins, P.F.; Wetzel, A.; Kereš, D.; Faucher-Giguère, C.A.; Quataert, E.; Boylan-Kolchin, M.; Murray, N.; Hayward, C.C.; Garrison-Kimmel, S.; Hummels, C.; et al. FIRE-2 simulations: physics versus numerics in galaxy formation. Mon. Not. R. Astron. Soc. 2018, 480, 800-863. doi: 10.1093/mnras/sty1690. [CrossRef]

59. Chan, T.K.; Kereš, D.; Oñorbe, J.; Hopkins, P.F.; Muratov, A.L.; Faucher-Giguère, C.A.; Quataert, E. The impact of baryonic physics on the structure of dark matter haloes: the view from the FIRE cosmological simulations. Mon. Not. R. Astron. Soc. 2015, 454, 2981-3001. doi: 10.1093/mnras/stv2165. [CrossRef]

60. Fitts, A.; Boylan-Kolchin, M.; Elbert, O.D.; Bullock, J.S.; Hopkins, P.F.; Oñorbe, J.; Wetzel, A.; Wheeler, C.; Faucher-Giguère, C.A.; Kereš, D.; et al. fire in the field: simulating the threshold of galaxy formation. Mon. Not. R. Astron. Soc. 2017, 471, 3547-3562. doi: 10.1093/mnras/stx1757. [CrossRef]

61. Wetzel, A.R.; Hopkins, P.F.; Kim, J.h.; Faucher-Giguère, C.A.; Kereš, D.; Quataert, E. Reconciling Dwarf Galaxies with $\Lambda$ CDM Cosmology: Simulating a Realistic Population of Satellites around a Milky Way-mass Galaxy. Astrophys. J. Lett. 2016, 827, L23. doi: $10.3847 / 2041-8205 / 827 / 2 /$ L23. [CrossRef]

62. Oñorbe, J.; Boylan-Kolchin, M.; Bullock, J.S.; Hopkins, P.F.; Kereš, D.; Faucher-Giguère, C.A.; Quataert, E.; Murray, N. Forged in FIRE: cusps, cores and baryons in low-mass dwarf galaxies. Mon. Not. R. Astron. Soc. 2015, 454, $2092-2106 . \quad$ doi: 10.1093/mnras/stv2072. [CrossRef]

63. Garrison-Kimmel, S.; Wetzel, A.; Bullock, J.S.; Hopkins, P.F.; Boylan-Kolchin, M.; Faucher-Giguère, C.A.; Kereš, D.; Quataert, E.; Sanderson, R.E.; Graus, A.S.; et al. Not so lumpy after all: modelling the depletion of dark matter subhaloes by Milky Way-like galaxies. Mon. Not. R. Astron. Soc. 2017, 471, 1709-1727. doi: 10.1093/mnras/stx1710. [CrossRef]

64. Peirani, S.; Dubois, Y.; Volonteri, M.; Devriendt, J.; Bundy, K.; Silk, J.; Pichon, C.; Kaviraj, S.; Gavazzi, R.; Habouzit, M. Density profile of dark matter haloes and galaxies in the HORIZON-AGN simulation: the impact of AGN feedback. Mon. Not. R. Astron. Soc. 2017, 472, 2153-2169. doi: 10.1093/mnras/stx2099. [CrossRef] 
65. Schaye, J.; Dalla Vecchia, C.; Booth, C.M.; Wiersma, R.P.C.; Theuns, T.; Haas, M.R.; Bertone, S.; Duffy, A.R.; McCarthy, I.G.; van de Voort, F. The physics driving the cosmic star formation history. Mon. Not. R. Astron. Soc. 2010, 402, 1536-1560. doi: 10.1111/j.1365-2966.2009.16029.x. [CrossRef]

66. Duffy, A.R.; Schaye, J.; Kay, S.T.; Dalla Vecchia, C.; Battye, R.A.; Booth, C.M. Impact of baryon physics on dark matter structures: A detailed simulation study of halo density profiles. Mon. Not. R. Astron. Soc. 2010, 405, 2161-2178. doi: 10.1111/j.13652966.2010.16613.x. [CrossRef]

67. Sawala, T.; Frenk, C.S.; Fattahi, A.; Navarro, J.F.; Bower, R.G.; Crain, R.A.; Dalla Vecchia, C.; Furlong, M.; Helly, J.C.; Jenkins, A.; et al. The APOSTLE simulations: solutions to the Local Group's cosmic puzzles. Mon. Not. R. Astron. Soc. 2016, 457, 1931-1943. doi: 10.1093/mnras/stw145. [CrossRef]

68. Fattahi, A.; Navarro, J.F.; Sawala, T.; Frenk, C.S.; Oman, K.A.; Crain, R.A.; Furlong, M.; Schaller, M.; Schaye, J.; Theuns, T.; et al. The APOSTLE project: Local Group kinematic mass constraints and simulation candidate selection. Mon. Not. R. Astron. Soc. 2016, 457, 844-856. doi: 10.1093/mnras/stv2970. [CrossRef]

69. Bose, S.; Frenk, C.S.; Jenkins, A.; Fattahi, A.; Gómez, F.A.; Grand , R.J.J.; Marinacci, F.; Navarro, J.F.; Oman, K.A.; Pakmor, R.; et al. No cores in dark matter-dominated dwarf galaxies with bursty star formation histories. Mon. Not. R. Astron. Soc. 2019, 486, 4790-4804. doi: 10.1093/mnras/stz1168. [CrossRef]

70. Benítez-Llambay, A.; Frenk, C.S.; Ludlow, A.D.; Navarro, J.F. Baryon-induced dark matter cores in the EAGLE simulations. Mon. Not. R. Astron. Soc. 2019, 488, 2387-2404. doi: 10.1093/mnras/stz1890. [CrossRef]

71. Power, C.; Navarro, J.F.; Jenkins, A.; Frenk, C.S.; White, S.D.M.; Springel, V.; Stadel, J.; Quinn, T. The inner structure of $\Lambda$ CDM haloes-I. A numerical convergence study. Mon. Not. R. Astron. Soc. 2003, 338, 14-34. doi: 10.1046/j.1365-8711.2003.05925.x. [CrossRef]

72. Bullock, J.S.; Boylan-Kolchin, M. Small-Scale Challenges to the $\Lambda$ CDM Paradigm. Annu. Rev. Astron. Astrophys. 2017, 55, 343-387. doi: 10.1146/annurev-astro-091916-055313. [CrossRef]

73. Dutton, A.A.; Macciò, A.V.; Buck, T.; Dixon, K.L.; Blank, M.; Obreja, A. NIHAO XX: The impact of the star formation threshold on the cusp-core transformation of cold dark matter haloes. Mon. Not. R. Astron. Soc. 2019, 486, 655-671. doi: 10.1093/mnras/stz889. [CrossRef]

74. Wang, L.; Dutton, A.A.; Stinson, G.S.; Macciò, A.V.; Penzo, C.; Kang, X.; Keller, B.W.; Wadsley, J. NIHAO project-I. Reproducing the inefficiency of galaxy formation across cosmic time with a large sample of cosmological hydrodynamical simulations. Mon. Not. R. Astron. Soc. 2015, 454, 83-94. doi: 10.1093/mnras/stv1937. [CrossRef]

75. Read, J.I.; Agertz, O.; Collins, M.L.M. Dark matter cores all the way down. Mon. Not. R. Astron. Soc. 2016, 459, 2573-2590. doi: 10.1093/mnras/stw713. [CrossRef]

76. Springel, V.; Pakmor, R.; Pillepich, A.; Weinberger, R.; Nelson, D.; Hernquist, L.; Vogelsberger, M.; Genel, S.; Torrey, P.; Marinacci, F.; et al. First results from the IllustrisTNG simulations: matter and galaxy clustering. Mon. Not. R. Astron. Soc. 2018, 475, 676-698. doi: 10.1093/mnras/stx3304. [CrossRef]

77. Oman, K.A.; Navarro, J.F.; Fattahi, A.; Frenk, C.S.; Sawala, T.; White, S.D.M.; Bower, R.; Crain, R.A.; Furlong, M.; Schaller, M.; et al. The unexpected diversity of dwarf galaxy rotation curves. Mon. Not. R. Astron. Soc. 2015, 452, 3650-3665. doi: 10.1093/mnras/stv1504. [CrossRef]

78. Peñarrubia, J.; Pontzen, A.; Walker, M.G.; Koposov, S.E. The Coupling between the Core/Cusp and Missing Satellite Problems. Astrophys. J. Lett. 2012, 759, L42. doi: 10.1088/2041-8205/759/2/L42. [CrossRef]

79. Revaz, Y.; Jablonka, P. Pushing back the limits: Detailed properties of dwarf galaxies in a $\Lambda$ CDM universe. arXiv 2018, arXiv:1801.06222. http://doi.org/10.1051/0004-6361/201832669.

80. Garrison-Kimmel, S.; Rocha, M.; Boylan-Kolchin, M.; Bullock, J.S.; Lally, J. Can feedback solve the too-big-to-fail problem? Mon. Not. R. Astron. Soc. 2013, 433, 3539-3546. doi: 10.1093/mnras/stt984. [CrossRef]

81. de Blok, W.J.G. The Core-Cusp Problem. Adv. Astron. 2010, 2010, 789293. doi: 10.1155/2010/789293. [CrossRef]

82. Genina, A.; Benítez-Llambay, A.; Frenk, C.S.; Cole, S.; Fattahi, A.; Navarro, J.F.; Oman, K.A.; Sawala, T.; Theuns, T. The core-cusp problem: A matter of perspective. Mon. Not. R. Astron. Soc. 2018, 474, 1398-1411. doi: 10.1093/mnras/stx2855. [CrossRef]

83. de Martino, I.; Chakrabarty, S.S.; Cesare, V.; Gallo, A.; Ostorero, L.; Diaferio, A. Dark Matters on the Scale of Galaxies. Universe 2020, 6, 107. doi: 10.3390/universe6080107. [CrossRef]

84. Bertone, G.; Hooper, D. History of dark matter. Rev. Mod. Phys. 2018, 90, 045002. doi: 10.1103/RevModPhys.90.045002 [CrossRef]

85. Klypin, A.; Kravtsov, A.V.; Valenzuela, O.; Prada, F. Where Are the Missing Galactic Satellites? Astrophys. J. 1999, 522, 82-92. doi: 10.1086/307643. [CrossRef]

86. Schneider, M.D.; Frenk, C.S.; Cole, S. The shapes and alignments of dark matter halos. J. Cosmol. Astropart. Phys. 2012, 2012, 030 doi: 10.1088/1475-7516/2012/05/030. [CrossRef]

87. Boylan-Kolchin, M.; Bullock, J.S.; Kaplinghat, M. Too big to fail? The puzzling darkness of massive Milky Way subhaloes. Mon. Not. R. Astron. Soc. 2011, 415, L40-L44. doi: 10.1111/j.1745-3933.2011.01074.x. [CrossRef]

88. Battaglia, G.; Helmi, A.; Breddels, M. Internal kinematics and dynamical models of dwarf spheroidal galaxies around the Milky Way. New Astron. Rev. 2013, 57, 52-79. doi: 10.1016/j.newar.2013.05.003. [CrossRef]

89. Walker, M. Dark Matter in the Galactic Dwarf Spheroidal Satellites. In Planets, Stars and Stellar Systems; Oswalt, T.D., Gilmore, G., Eds.; Springer Science+Business Media: Dordrecht, The Netherlands, 2013; Volume 5, p. 1039, ISBN 978-94-007-5611-3. doi: 10.1007/978-94-007-5612-0_20. [CrossRef] 
90. Hammer, F.; Yang, Y.; Arenou, F.; Babusiaux, C.; Wang, J.; Puech, M.; Flores, H. Galactic Forces Rule the Dynamics of Milky Way Dwarf Galaxies. Astrophys. J. 2018, 860, 76. doi: 10.3847/1538-4357/aac3da. [CrossRef]

91. Errani, R.; Peñarrubia, J.; Walker, M.G. Systematics in virial mass estimators for pressure-supported systems. Mon. Not. R. Astron. Soc. 2018, 481, 5073-5090. doi: 10.1093/mnras/sty2505. [CrossRef]

92. Read, J.I.; Walker, M.G.; Steger, P. Dark matter heats up in dwarf galaxies. Mon. Not. R. Astron. Soc. 2019, 484, 1401-1420. doi: 10.1093/mnras/sty3404. [CrossRef]

93. Binney, J. The radius-dependence of velocity dispersion in elliptical galaxies. Mon. Not. R. Astron. Soc. 1980, 190, 873-880. doi: 10.1093/mnras/190.4.873. [CrossRef]

94. Binney, J.; Tremaine, S. Galactic Dynamics, 2nd ed.; Princeton University Press: Princeton, NJ, USA, 2008.

95. Binney, J.; Mamon, G.A. M/L and velocity anisotropy from observations of spherical galaxies, of must M 87 have a massive black hole ? Mon. Not. R. Astron. Soc. 1982, 200, 361-375. doi: 10.1093/mnras/200.2.361. [CrossRef]

96. Walker, M.G.; Mateo, M.; Olszewski, E.W.; Peñarrubia, J.; Evans, N.W.; Gilmore, G. A Universal Mass Profile for Dwarf Spheroidal Galaxies? Astrophys. J. 2009, 704, 1274-1287. doi: 10.1088/0004-637X/704/2/1274. [CrossRef]

97. Read, J.I.; Steger, P. How to break the density-anisotropy degeneracy in spherical stellar systems. Mon. Not. R. Astron. Soc. 2017, 471, 4541-4558. doi: 10.1093/mnras/stx1798. [CrossRef]

98. Genina, A.; Read, J.I.; Frenk, C.S.; Cole, S.; Benitez-Llambay, A.; Ludlow, A.D.; Navarro, J.F.; Oman, K.A.; Robertson, A. To beta or not to beta: Can higher-order Jeans analysis break the mass-anisotropy degeneracy in simulated dwarfs? arXiv 2019, arXiv:1911.09124.

99. Merrifield, M.R.; Kent, S.M. Fourth Moments and the Dynamics of Spherical Systems. Astron. J. 1990, $99,1548 . \quad$ doi: 10.1086/115438. [CrossRef]

100. Evans, N.W.; An, J.; Walker, M.G. Cores and cusps in the dwarf spheroidals. Mon. Not. R. Astron. Soc. 2009, 393, L50-L54. doi: 10.1111/j.1745-3933.2008.00596.x. [CrossRef]

101. Gilmore, G.; Wilkinson, M.I.; Wyse, R.F.G.; Kleyna, J.T.; Koch, A.; Evans, N.W.; Grebel, E.K. The Observed Properties of Dark Matter on Small Spatial Scales. Astrophys. J. 2007, 663, 948-959. doi: 10.1086/518025. [CrossRef]

102. Walker, M.G.; Peñarrubia, J. A Method for Measuring (Slopes of) the Mass Profiles of Dwarf Spheroidal Galaxies. Astrophys. J. 2011, 742, 20. doi: 10.1088/0004-637X/742/1/20. [CrossRef]

103. Agnello, A.; Evans, N.W. A Virial Core in the Sculptor Dwarf Spheroidal Galaxy. Astrophys. J. Lett. 2012, 754, L39. doi: 10.1088/2041-8205/754/2/L39. [CrossRef]

104. Strigari, L.E.; Frenk, C.S.; White, S.D.M. Kinematics of Milky Way satellites in a Lambda cold dark matter universe. Mon. Not. R. Astron. Soc. 2010, 408, 2364-2372. doi: 10.1111/j.1365-2966.2010.17287.x. [CrossRef]

105. Breddels, M.A.; Helmi, A.; van den Bosch, R.C.E.; van de Ven, G.; Battaglia, G. Orbit-based dynamical models of the Sculptor dSph galaxy. Mon. Not. R. Astron. Soc. 2013, 433, 3173-3189. doi: 10.1093/mnras/stt956. [CrossRef]

106. Richardson, T.; Fairbairn, M. On the dark matter profile in Sculptor: breaking the $\beta$ degeneracy with Virial shape parameters. Mon. Not. R. Astron. Soc. 2014, 441, 1584-1600. doi: 10.1093/mnras/stu691. [CrossRef]

107. Battaglia, G.; Helmi, A.; Tolstoy, E.; Irwin, M.; Hill, V.; Jablonka, P. The Kinematic Status and Mass Content of the Sculptor Dwarf Spheroidal Galaxy. Astrophys. J. Lett. 2008, 681, L13. doi: 10.1086/590179. [CrossRef]

108. Łokas, E.L. The mass and velocity anisotropy of the Carina, Fornax, Sculptor and Sextans dwarf spheroidal galaxies. Mon. Not. R. Astron. Soc. 2009, 394, L102-L106. doi: 10.1111/j.1745-3933.2009.00620.x. [CrossRef]

109. Jardel, J.R.; Gebhardt, K.; Fabricius, M.H.; Drory, N.; Williams, M.J. Measuring Dark Matter Profiles Non-Parametrically in Dwarf Spheroidals: An Application to Draco. Astrophys. J. 2013, 763, 91. doi: 10.1088/0004-637X/763/2/91. [CrossRef]

110. Wilkinson, M.I.; Kleyna, J.; Evans, N.W.; Gilmore, G. Dark matter in dwarf spheroidals-I. Models. Mon. Not. R. Astron. Soc. 2002, 330, 778-791. doi: 10.1046/j.1365-8711.2002.05154.x. [CrossRef]

111. Strigari, L.E.; Bullock, J.S.; Kaplinghat, M. Determining the Nature of Dark Matter with Astrometry. Astrophys. J. Lett. 2007, 657, L1-L4. doi: 10.1086/512976. [CrossRef]

112. Massari, D.; Breddels, M.A.; Helmi, A.; Posti, L.; Brown, A.G.A.; Tolstoy, E. Three-dimensional motions in the Sculptor dwarf galaxy as a glimpse of a new era. Nat. Astron. 2018, 2, 156-161. doi: 10.1038/s41550-017-0322-y. [CrossRef]

113. Lazar, A.; Bullock, J.S. Accurate mass estimates from the proper motions of dispersion-supported galaxies. Mon. Not. R. Astron. Soc. 2020, 493, 5825-5837. doi: 10.1093/mnras/staa692. [CrossRef]

114. Hayashi, K.; Chiba, M.; Ishiyama, T. Diversity of Dark Matter Density Profiles in the Galactic Dwarf Spheroidal Satellites. Astrophys. J. 2020, 904, 45. doi: 10.3847/1538-4357/abbe0a. [CrossRef]

115. Irwin, M.; Hatzidimitriou, D. Structural parameters for the Galactic dwarf spheroidals. Mon. Not. R. Astron. Soc. 1995, 277, 1354-1378. doi: 10.1093/mnras/277.4.1354. [CrossRef]

116. Vera-Ciro, C.A.; Sales, L.V.; Helmi, A.; Navarro, J.F. The shape of dark matter subhaloes in the Aquarius simulations. Mon. Not. R. Astron. Soc. 2014, 439, 2863-2872. doi: 10.1093/mnras/stu153. [CrossRef]

117. Kuhlen, M.; Diemand, J.; Madau, P. The Shapes, Orientation, and Alignment of Galactic Dark Matter Subhalos. Astrophys. J. 2007, 671, 1135-1146. doi: 10.1086/522878. [CrossRef]

118. Jing, Y.P.; Suto, Y. Triaxial Modeling of Halo Density Profiles with High-Resolution N-Body Simulations. Astrophys. J. 2002, 574, 538-553. doi: 10.1086/341065. [CrossRef]

119. Hayashi, K.; Chiba, M. Structural Properties of Non-spherical Dark Halos in Milky Way and Andromeda Dwarf Spheroidal Galaxies. Astrophys. J. 2015, 810, 22. doi: 10.1088/0004-637X/810/1/22. [CrossRef] 
120. Gnedin, O.Y.; Ostriker, J.P. On the Self-consistent Response of Stellar Systems to Gravitational Shocks. Astrophys. J. 1999, 513, 626-637. doi: 10.1086/306864. [CrossRef]

121. Gnedin, O.Y.; Lee, H.M.; Ostriker, J.P. Effects of Tidal Shocks on the Evolution of Globular Clusters. Astrophys. J. 1999, 522, 935-949. doi: 10.1086/307659. [CrossRef]

122. Spitzer, L. Dynamical evolution of globular clusters. Annu. Rev. Astron. Astrophys. 1987, 25, 565-601.

123. Hammer, F.; Yang, Y.; Wang, J.; Arenou, F.; Puech, M.; Flores, H.; Babusiaux, C. On the Absence of Dark Matter in Dwarf Galaxies Surrounding the Milky Way. Astrophys. J. 2019, 883, 171. doi: 10.3847/1538-4357/ab36b6. [CrossRef]

124. Hammer, F.; Yang, Y.; Arenou, F.; Wang, J.; Li, H.; Bonifacio, P.; Babusiaux, C. Orbital Evidences for Dark-matter-free Milky Way Dwarf Spheroidal Galaxies. Astrophys. J. 2020, 892, 3. doi: 10.3847/1538-4357/ab77be. [CrossRef]

125. Gnedin, O.Y.; Hernquist, L.; Ostriker, J.P. Tidal Shocking by Extended Mass Distributions. Astrophys. J. 1999, 514, 109-118. doi: 10.1086/306910. [CrossRef]

126. Chang, L.J.; Necib, L. Dark matter density profiles in dwarf galaxies: linking Jeans modelling systematics and observation. Mon. Not. R. Astron. Soc. 2021, 507, 4715-4733. doi: 10.1093/mnras/stab2440. [CrossRef]

127. Amorisco, N.C.; Evans, N.W. Dark matter cores and cusps: the case of multiple stellar populations in dwarf spheroidals. Mon. Not. R. Astron. Soc. 2012, 419, 184-196. doi: 10.1111/j.1365-2966.2011.19684.x. [CrossRef]

128. Adams, J.J.; Simon, J.D.; Fabricius, M.H.; van den Bosch, R.C.E.; Barentine, J.C.; Bender, R.; Gebhardt, K.; Hill, G.J.; Murphy, J.D.; Swaters, R.A.; et al. Dwarf Galaxy Dark Matter Density Profiles Inferred from Stellar and Gas Kinematics. Astrophys. J. 2014, 789, 63. doi: 10.1088/0004-637X/789/1/63. [CrossRef]

129. Wolf, J.; Martinez, G.D.; Bullock, J.S.; Kaplinghat, M.; Geha, M.; Muñoz, R.R.; Simon, J.D.; Avedo, F.F. Accurate masses for dispersion-supported galaxies. Mon. Not. R. Astron. Soc. 2010, 406, 1220-1237. doi: 10.1111/j.1365-2966.2010.16753.x. [CrossRef]

130. Macciò, A.V.; Frings, J.; Buck, T.; Penzo, C.; Dutton, A.A.; Blank, M.; Obreja, A. The edge of galaxy formation-I. Formation and evolution of MW-satellite analogues before accretion. Mon. Not. R. Astron. Soc. 2017, 472, 2356-2366. doi: 10.1093/mnras/stx2048. [CrossRef]

131. Robles, V.H.; Bullock, J.S.; Elbert, O.D.; Fitts, A.; González-Samaniego, A.; Boylan-Kolchin, M.; Hopkins, P.F.; Faucher-Giguère, C.A.; Kereš, D.; Hayward, C.C. SIDM on FIRE: Hydrodynamical self-interacting dark matter simulations of low-mass dwarf galaxies. Mon. Not. R. Astron. Soc. 2017, 472, 2945-2954. doi: 10.1093/mnras/stx2253. [CrossRef] [PubMed]

132. Noh, Y.; McQuinn, M. A physical understanding of how reionization suppresses accretion on to dwarf haloes. Mon. Not. R. Astron. Soc. 2014, 444, 503-514. doi: 10.1093/mnras/stu1412. [CrossRef]

133. Bullock, J.S.; Kravtsov, A.V.; Weinberg, D.H. Reionization and the Abundance of Galactic Satellites. Astrophys. J. 2000, 539, 517-521. doi: 10.1086/309279. [CrossRef]

134. Efstathiou, G. Suppressing the formation of dwarf galaxies via photoionization. Mon. Not. R. Astron. Soc. 1992, 256, 43P-47P. doi: 10.1093/mnras/256.1.43P. [CrossRef]

135. Quinn, T.; Katz, N.; Efstathiou, G. Photoionization and the formation of dwarf galaxies. Mon. Not. R. Astron. Soc. 1996, 278, L49-L54. doi: 10.1093/mnras/278.4.L49. [CrossRef]

136. Burger, J.D.; Zavala, J. SN-driven mechanism of cusp-core transformation: An appraisal. arXiv 2021, arXiv:2103.01231.

137. Vogelsberger, M.; Zavala, J.; Simpson, C.; Jenkins, A. Dwarf galaxies in CDM and SIDM with baryons: Observational probes of the nature of dark matter. Mon. Not. R. Astron. Soc. 2014, 444, 3684-3698. doi: 10.1093/mnras/stu1713. [CrossRef]

138. Fry, A.B.; Governato, F.; Pontzen, A.; Quinn, T.R. Self Interacting Dark Matter and Baryons. American Astronomical Society Meeting Abstracts. 2015. Volume 225, p. 402. Available online: https://ui.adsabs.harvard.edu/abs/2015AAS...22540205F/ abstract (accessed on 27 October 2021).

139. Burger, J.D.; Zavala, J. The nature of core formation in dark matter haloes: Adiabatic or impulsive? Mon. Not. R. Astron. Soc. 2019, 485, 1008-1028. doi: 10.1093/mnras/stz496. [CrossRef]

140. Ceverino, D.; Klypin, A.; Klimek, E.S.; Trujillo-Gomez, S.; Churchill, C.W.; Primack, J.; Dekel, A. Radiative feedback and the low efficiency of galaxy formation in low-mass haloes at high redshift. Mon. Not. R. Astron. Soc. 2014, 442, 1545-1559. doi: 10.1093/mnras/stu956. [CrossRef]

141. Wheeler, C.; Hopkins, P.F.; Pace, A.B.; Garrison-Kimmel, S.; Boylan-Kolchin, M.; Wetzel, A.; Bullock, J.S.; Kereš, D.; FaucherGiguère, C.A.; Quataert, E. Be it therefore resolved: Cosmological simulations of dwarf galaxies with 30 solar mass resolution. Mon. Not. R. Astron. Soc. 2019, 490, 4447-4463. doi: 10.1093/mnras/stz2887. [CrossRef]

142. Zentner, A.R.; Bullock, J.S. Halo Substructure and the Power Spectrum. Astrophys. J. 2003, 598, 49-72. doi: 10.1086/378797. [CrossRef]

143. Colín, P.; Avila-Reese, V.; Valenzuela, O. Substructure and Halo Density Profiles in a Warm Dark Matter Cosmology. Astrophys. J. 2000, 542, 622-630. doi: 10.1086/317057. [CrossRef]

144. Goodman, J. Repulsive dark matter. New Astron. 2000, 5, 103-107. doi: 10.1016/S1384-1076(00)00015-4. [CrossRef]

145. Hu, W.; Barkana, R.; Gruzinov, A. Fuzzy Cold Dark Matter: The Wave Properties of Ultralight Particles. Phys. Rev. Lett. 2000, 85, 1158-1161. doi: 10.1103/PhysRevLett.85.1158. [CrossRef]

146. Kaplinghat, M.; Knox, L.; Turner, M.S. Annihilating Cold Dark Matter. Phys. Rev. Lett. 2000, 85, 3335-3338. doi: 10.1103/PhysRevLett.85.3335. [CrossRef]

147. Peebles, P.J.E. Fluid Dark Matter. Astrophys. J. Lett. 2000, 534, L127-L129. doi: 10.1086/312677. [CrossRef]

148. Sommer-Larsen, J.; Dolgov, A. Formation of Disk Galaxies: Warm Dark Matter and the Angular Momentum Problem. Astrophys. J. 2001, 551, 608-623. doi: 10.1086/320211. [CrossRef] 
149. Buchdahl, H.A. Non-linear Lagrangians and cosmological theory. Mon. Not. R. Astron. Soc. 1970, $150,1$. mnras/150.1.1. [CrossRef]

150. Godani, N.; Samanta, G.C. Traversable wormholes in $\mathrm{f}(\mathrm{R})$ gravity with constant and variable redshift functions. New Astron. 2020, 80, 101399. doi: 10.1016/j.newast.2020.101399. [CrossRef]

151. Bengochea, G.R.; Ferraro, R. Dark torsion as the cosmic speed-up. Phys. Rev. D 2009, $79,124019$. 79.124019. [CrossRef]

152. Linder, E.V. Einstein's other gravity and the acceleration of the Universe. Phys. Rev. D 2010, 81, 127301. PhysRevD.81.12730. [CrossRef]

153. Dent, J.B.; Dutta, S.; Saridakis, E.N. f(T) gravity mimicking dynamical dark energy. Background and perturbation analysis. J. Cosmol. Astropart. Phys. 2011, 2011, 009. doi: 10.1088/1475-7516/2011/01/009. [CrossRef]

154. Zheng, R.; Huang, Q.G. Growth factor in f(T) gravity. J. Cosmol. Astropart. Phys. 2011, 2011, 002. doi: 10.1088/14757516/2011/03/002. [CrossRef]

155. Haghi, H.; Amiri, V. Testing modified gravity with dwarf spheroidal galaxies. Mon. Not. R. Astron. Soc. 2016, 463, 1944-1951. doi: 10.1093/mnras/stw2140. [CrossRef]

156. de Martino, I. Giant low-surface-brightness dwarf galaxy as a test bench for MOdified Gravity. Mon. Not. R. Astron. Soc. 2020, 493, 2373-2376. doi: 10.1093/mnras/staa460. [CrossRef]

157. Haghi, H.; Khodadadi, A.; Ghari, A.; Zonoozi, A.H.; Kroupa, P. Rotation curves of galaxies and the stellar mass-to-light ratio. Mon. Not. R. Astron. Soc. 2018, 477, 4187-4199. doi: 10.1093/mnras/sty523. [CrossRef]

158. Milgrom, M. A modification of the Newtonian dynamics as a possible alternative to the hidden mass hypothesis. Astrophys. J. 1983, 270, 365-370. doi: 10.1086/161130. [CrossRef]

159. Famaey, B.; McGaugh, S.S. Modified Newtonian Dynamics (MOND): Observational Phenomenology and Relativistic Extensions. Living Rev. Relativ. 2012, 15, 10. doi: 10.12942/lrr-2012-10. [CrossRef]

160. Angus, G.W. Dwarf spheroidals in MOND. Mon. Not. R. Astron. Soc. 2008, 387, 1481-1488. doi: 10.1111/j.1365-2966.2008.13351.x. [CrossRef]

161. Angus, G.W.; Gentile, G.; Diaferio, A.; Famaey, B.; van der Heyden, K.J. N-body simulations of the Carina dSph in MOND. Mon. Not. R. Astron. Soc. 2014, 440, 746-761. doi: 10.1093/mnras/stu182. [CrossRef]

162. Chandrasekhar, S. Dynamical Friction. I. General Considerations: The Coefficient of Dynamical Friction. Astrophys. J. 1943, 97, 255. doi: 10.1086/144517. [CrossRef]

163. Petts, J.A.; Read, J.I.; Gualandris, A. A semi-analytic dynamical friction model for cored galaxies. Mon. Not. R. Astron. Soc. 2016, 463, 858-869. doi: 10.1093/mnras/stw2011. [CrossRef]

164. El-Zant, A.; Shlosman, I.; Hoffman, Y. Dark Halos: The Flattening of the Density Cusp by Dynamical Friction. Astrophys. J. 2001, 560, 636-643. doi: 10.1086/322516. [CrossRef]

165. Boldrini, P.; Mohayaee, R.; Silk, J. Flattening of Dark Matter Cusps during Mergers: Model of M31. Astrophys. J. 2021, 919, 86. doi: 10.3847/1538-4357/ac12d3. [CrossRef]

166. Goerdt, T.; Moore, B.; Read, J.I.; Stadel, J. Core Creation in Galaxies and Halos Via Sinking Massive Objects. Astrophys. J. 2010, 725, 1707-1716. doi: 10.1088/0004-637X/725/2/1707. [CrossRef]

167. Read, J.I.; Wilkinson, M.I.; Evans, N.W.; Gilmore, G.; Kleyna, J.T. The tidal stripping of satellites. Mon. Not. R. Astron. Soc. 2006, 366, 429-437. doi: 10.1111/j.1365-2966.2005.09861.x. [CrossRef]

168. Fitts, A.; Boylan-Kolchin, M.; Bullock, J.S.; Weisz, D.R.; El-Badry, K.; Wheeler, C.; Faucher-Giguère, C.A.; Quataert, E.; Hopkins, P.F.; Kereš, D.; et al. No assembly required: Mergers are mostly irrelevant for the growth of low-mass dwarf galaxies. Mon. Not. R. Astron. Soc. 2018, 479, 319-331. doi: 10.1093/mnras/sty1488. [CrossRef]

169. Diemand, J.; Kuhlen, M.; Madau, P.; Zemp, M.; Moore, B.; Potter, D.; Stadel, J. Clumps and streams in the local dark matter distribution. Nature 2008, 454, 735-738. doi: 10.1038/nature07153. [CrossRef]

170. Springel, V.; Wang, J.; Vogelsberger, M.; Ludlow, A.; Jenkins, A.; Helmi, A.; Navarro, J.F.; Frenk, C.S.; White, S.D.M. The Aquarius Project: The subhaloes of galactic haloes. Mon. Not. R. Astron. Soc. 2008, 391, 1685-1711. doi: 10.1111/j.1365-2966.2008.14066.x. [CrossRef]

171. Banik, N.; Bovy, J.; Bertone, G.; Erkal, D.; de Boer, T.J.L. Evidence of a population of dark subhaloes from Gaia and Pan-STARRS observations of the GD-1 stream. Mon. Not. R. Astron. Soc. 2021, 502, 2364-2380. doi: 10.1093/mnras/stab210. [CrossRef]

172. Zavala, J.; Frenk, C.S. Dark Matter Haloes and Subhaloes. Galaxies 2019, 7, 81. doi: 10.3390/galaxies7040081. [CrossRef]

173. Boldrini, P.; Mohayaee, R.; Silk, J. Subhalo sinking and off-centre massive black holes in dwarf galaxies. Mon. Not. R. Astron. Soc. 2020, 495, L12-L16. doi: 10.1093/mnrasl/slaa043. [CrossRef]

174. Wetzel, A.R. On the orbits of infalling satellite haloes. Mon. Not. R. Astron. Soc. 2011, 412, 49-58. doi: 10.1111/j.13652966.2010.17877.x. [CrossRef]

175. Orkney, M.D.A.; Read, J.I.; Rey, M.P.; Nasim, I.; Pontzen, A.; Agertz, O.; Kim, S.Y.; Delorme, M.; Dehnen, W. EDGE: Two routes to dark matter core formation in ultra-faint dwarfs. Mon. Not. R. Astron. Soc. 2021, 504, 3509-3522. doi: 10.1093/mnras/stab1066. [CrossRef]

176. Leung, G.Y.C.; Leaman, R.; van de Ven, G.; Battaglia, G. A dwarf-dwarf merger and dark matter core as a solution to the globular cluster problems in the Fornax dSph. Mon. Not. R. Astron. Soc. 2020, 493, 320-336. doi: 10.1093/mnras/stz3017. [CrossRef]

177. Nipoti, C.; Binney, J. Early flattening of dark matter cusps in dwarf spheroidal galaxies. Mon. Not. R. Astron. Soc. 2015, 446, 1820-1828. doi: 10.1093/mnras/stu2217. [CrossRef] 
178. Cole, D.R.; Dehnen, W.; Wilkinson, M.I. Weakening dark matter cusps by clumpy baryonic infall. Mon. Not. R. Astron. Soc. 2011, 416, 1118-1134. doi: 10.1111/j.1365-2966.2011.19110.x. [CrossRef]

179. Del Popolo, A.; Le Delliou, M. A unified solution to the small scale problems of the $\Lambda$ CDM model II: Introducing parent-satellite interaction. J. Cosmol. Astropart. Phys. 2014, 2014, 051. doi: 10.1088/1475-7516/2014/12/051. [CrossRef]

180. Inoue, S.; Saitoh, T.R. Cores and revived cusps of dark matter haloes in disc galaxy formation through clump clusters. Mon. Not. R. Astron. Soc. 2011, 418, 2527-2531. doi: 10.1111/j.1365-2966.2011.19873.x. [CrossRef]

181. Peebles, P.J.E. Dark matter and the origin of galaxies and globular star clusters. Astrophys. J. 1984, 277, 470-477. doi: 10.1086/161714. [CrossRef]

182. Bromm, V.; Clarke, C.J. The Formation of the First Globular Clusters in Dwarf Galaxies before the Epoch of Reionization. Astrophys. J. Lett. 2002, 566, L1-L4. doi: 10.1086/339440. [CrossRef]

183. Mashchenko, S.; Sills, A. Globular Clusters with Dark Matter Halos. II. Evolution in a Tidal Field. Astrophys. J. 2005, 619, 258-269. doi: 10.1086/426133. [CrossRef]

184. Ricotti, M.; Parry, O.H.; Gnedin, N.Y. A Common Origin for Globular Clusters and Ultra-faint Dwarfs in Simulations of the First Galaxies. Astrophys. J. 2016, 831, 204, doi: 10.3847/0004-637X/831/2/204. [CrossRef]

185. Conroy, C.; Loeb, A.; Spergel, D.N. Evidence against Dark Matter Halos Surrounding the Globular Clusters MGC1 and NGC 2419. Astrophys. J. 2011, 741, 72. doi: 10.1088/0004-637X/741/2/72. [CrossRef]

186. Ibata, R.; Nipoti, C.; Sollima, A.; Bellazzini, M.; Chapman, S.C.; Dalessandro, E. Do globular clusters possess dark matter haloes? A case study in NGC 2419. Mon. Not. R. Astron. Soc. 2013, 428, 3648-3659. doi: 10.1093/mnras/sts302. [CrossRef]

187. Shin, J.; Kim, S.S.; Lee, Y.W. Dark Matter Content in Globular Cluster NGC 6397. J. Korean Astron. Soc. 2013, 46, 173-181. doi: 10.5303/JKAS.2013.46.4.173. [CrossRef]

188. Moore, B. Constraints on the Global Mass-to-Light Ratios and on the Extent of Dark Matter Halos in Globular Clusters and Dwarf Spheroidals. Astrophys. J. Lett. 1996, 461, L13. doi: 10.1086/309998. [CrossRef]

189. Baumgardt, H.; Côté, P.; Hilker, M.; Rejkuba, M.; Mieske, S.; Djorgovski, S.G.; Stetson, P. The velocity dispersion and mass-to-light ratio of the remote halo globular cluster NGC2419. Mon. Not. R. Astron. Soc. 2009, 396, 2051-2060. doi: 10.1111/j.13652966.2009.14932.x. [CrossRef]

190. Lane, R.R.; Kiss, L.L.; Lewis, G.F.; Ibata, R.A.; Siebert, A.; Bedding, T.R.; Székely, P.; Balog, Z.; Szabó, G.M. Halo globular clusters observed with AAOmega: Dark matter content, metallicity and tidal heating. Mon. Not. R. Astron. Soc. 2010, 406, 2732-2742. doi: 10.1111/j.1365-2966.2010.16874.x. [CrossRef]

191. Hurst, T.J.; Zentner, A.R.; Natarajan, A.; Badenes, C. Indirect probes of dark matter and globular cluster properties from dark matter annihilation within the coolest white dwarfs. Phys. Rev. D 2015, 91, 103514. doi: 10.1103/PhysRevD.91.103514. [CrossRef]

192. Peter, A.H.G.; Moody, C.E.; Kamionkowski, M. Dark-matter decays and self-gravitating halos. Phys. Rev. D 2010, 81, 103501. doi: 10.1103/PhysRevD.81.103501. [CrossRef]

193. Davis, A.J.; Khochfar, S.; Dalla Vecchia, C. The First Billion Years project: dark matter haloes going from contraction to expansion and back again. Mon. Not. R. Astron. Soc. 2014, 443, 985-1001. doi: 10.1093/mnras/stu1201. [CrossRef]

194. Saitoh, T.R.; Koda, J.; Okamoto, T.; Wada, K.; Habe, A. Tidal Disruption of Dark Matter Halos around Proto-Globular Clusters. Astrophys. J. 2006, 640, 22-30. doi: 10.1086/500104. [CrossRef]

195. Bekki, K.; Yong, D. On the origin of the stellar halo and multiple stellar populations in the globular cluster NGC 1851. Mon. Not. R. Astron. Soc. 2012, 419, 2063-2076. doi: 10.1111/j.1365-2966.2011.19856.x. [CrossRef]

196. Pillepich, A.; Springel, V.; Nelson, D.; Genel, S.; Naiman, J.; Pakmor, R.; Hernquist, L.; Torrey, P.; Vogelsberger, M.; Weinberger, R.; et al. Simulating galaxy formation with the IllustrisTNG model. Mon. Not. R. Astron. Soc. 2018, 473, 4077-4106. doi: 10.1093/mnras/stx2656. [CrossRef]

197. Boldrini, P.; Mohayaee, R.; Silk, J. Embedding globular clusters in dark matter minihaloes solves the cusp-core and timing problems in the Fornax dwarf galaxy. Mon. Not. R. Astron. Soc. 2020, 492, 3169-3178. doi: 10.1093/mnras/staa011. [CrossRef]

198. Angus, G.W.; Diaferio, A. Resolving the timing problem of the globular clusters orbiting the Fornax dwarf galaxy. Mon. Not. R. Astron. Soc. 2009, 396, 887-893. doi: 10.1111/j.1365-2966.2009.14745.x. [CrossRef]

199. Sanders, J.L.; Evans, N.W.; Dehnen, W. Tidal disruption of dwarf spheroidal galaxies: The strange case of Crater II. Mon. Not. R. Astron. Soc. 2018, 478, 3879-3889. doi: 10.1093/mnras/sty1278. [CrossRef]

200. Torrealba, G.; Belokurov, V.; Koposov, S.E.; Li, T.S.; Walker, M.G.; Sanders, J.L.; Geringer-Sameth, A.; Zucker, D.B.; Kuehn, K.; Evans, N.W.; et al. The hidden giant: discovery of an enormous Galactic dwarf satellite in Gaia DR2. Mon. Not. R. Astron. Soc. 2019, 488, 2743-2766. doi: 10.1093/mnras/stz1624. [CrossRef]

201. Frings, J.; Macciò, A.; Buck, T.; Penzo, C.; Dutton, A.; Blank, M.; Obreja, A. The edge of galaxy formation-II. Evolution of Milky Way satellite analogues after infall. Mon. Not. R. Astron. Soc. 2017, 472, 3378-3389. doi: 10.1093/mnras/stx2171. [CrossRef]

202. Genina, A.; Read, J.I.; Fattahi, A.; Frenk, C.S. Can tides explain the low dark matter density in Fornax? arXiv 2020, arXiv:2011.09482.

203. Walker, M.G.; Mateo, M.; Olszewski, E.W.; Bernstein, R.; Wang, X.; Woodroofe, M. Internal Kinematics of the Fornax Dwarf Spheroidal Galaxy. Astron. J. 2006, 131, 2114-2139. doi: 10.1086/500193. [CrossRef]

204. Wang, M.Y.; de Boer, T.; Pieres, A.; Li, T.S.; Drlica-Wagner, A.; Koposov, S.E.; Vivas, A.K.; Pace, A.B.; Santiago, B.; Walker, A.R.; et al. The Morphology and Structure of Stellar Populations in the Fornax Dwarf Spheroidal Galaxy from Dark Energy Survey Data. Astrophys. J. 2019, 881, 118. doi: 10.3847/1538-4357/ab31a9. [CrossRef] 
205. Laporte, C.F.P.; Penarrubia, J. Under the sword of Damocles: Plausible regeneration of dark matter cusps at the smallest galactic scales. Mon. Not. R. Astron. Soc. 2015, 449, L90-L94. doi: 10.1093/mnrasl/slv008. [CrossRef]

206. Dekel, A.; Devor, J.; Hetzroni, G. Galactic halo cusp-core: Tidal compression in mergers. Mon. Not. R. Astron. Soc. 2003, 341, 326-342. doi: 10.1046/j.1365-8711.2003.06432.x. [CrossRef]

207. Peñarrubia, J.; Benson, A.J.; Walker, M.G.; Gilmore, G.; McConnachie, A.W.; Mayer, L. The impact of dark matter cusps and cores on the satellite galaxy population around spiral galaxies. Mon. Not. R. Astron. Soc. 2010, 406, 1290-1305. doi: 10.1111/j.1365-2966.2010.16762.x. [CrossRef]

208. Dutton, A.A.; Macciò, A.V. Cold dark matter haloes in the Planck era: Evolution of structural parameters for Einasto and NFW profiles. Mon. Not. R. Astron. Soc. 2014, 441, 3359-3374. doi: 10.1093/mnras/stu742. [CrossRef]

209. Brook, C.B.; Stinson, G.; Gibson, B.K.; Wadsley, J.; Quinn, T. MaGICC discs: Matching observed galaxy relationships over a wide stellar mass range. Mon. Not. R. Astron. Soc. 2012, 424, 1275-1283. doi: 10.1111/j.1365-2966.2012.21306.x. [CrossRef]

210. Teyssier, R.; Pontzen, A.; Dubois, Y.; Read, J.I. Cusp-core transformations in dwarf galaxies: Observational predictions. Mon. Not. R. Astron. Soc. 2013, 429, 3068-3078. doi: 10.1093/mnras/sts563. [CrossRef]

211. Madau, P.; Shen, S.; Governato, F. Dark Matter Heating and Early Core Formation in Dwarf Galaxies. Astrophys. J. Lett. 2014, 789, L17. doi: 10.1088/2041-8205/789/1/L17. [CrossRef]

212. McGaugh, S. Predictions and Outcomes for the Dynamics of Rotating Galaxies. Galaxies 2020, 8,35 galaxies8020035. [CrossRef]

213. Bode, P.; Ostriker, J.P.; Turok, N. Halo Formation in Warm Dark Matter Models. Astrophys. J. 2001, 556, $93-107 . \quad$ doi: 10.1086/321541. [CrossRef]

214. Schneider, A.; Smith, R.E.; Macciò, A.V.; Moore, B. Non-linear evolution of cosmological structures in warm dark matter models. Mon. Not. R. Astron. Soc. 2012, 424, 684-698. doi: 10.1111/j.1365-2966.2012.21252.x. [CrossRef]

215. Macciò, A.V.; Paduroiu, S.; Anderhalden, D.; Schneider, A.; Moore, B. Cores in warm dark matter haloes: A Catch 22 problem. Mon. Not. R. Astron. Soc. 2012, 424, 1105-1112. doi: 10.1111/j.1365-2966.2012.21284.x. [CrossRef]

216. Shao, S.; Gao, L.; Theuns, T.; Frenk, C.S. The phase-space density of fermionic dark matter haloes. Mon. Not. R. Astron. Soc. 2013, 430, 2346-2357. doi: 10.1093/mnras/stt053. [CrossRef]

217. Lovell, M.R.; Frenk, C.S.; Eke, V.R.; Jenkins, A.; Gao, L.; Theuns, T. The properties of warm dark matter haloes. Mon. Not. R. Astron. Soc. 2014, 439, 300-317. doi: 10.1093/mnras/stt2431. [CrossRef]

218. Kochanek, C.S.; White, M. A Quantitative Study of Interacting Dark Matter in Halos. Astrophys. J. 2000, 543, 514-520. doi: 10.1086/317149. [CrossRef]

219. Miralda-Escudé, J. A Test of the Collisional Dark Matter Hypothesis from Cluster Lensing. Astrophys. J. 2002, 564, 60-64. doi: 10.1086/324138. [CrossRef]

220. Zavala, J.; Vogelsberger, M.; Walker, M.G. Constraining self-interacting dark matter with the Milky way's dwarf spheroidals. Mon. Not. R. Astron. Soc. 2013, 431, L20-L24. doi: 10.1093/mnrasl/sls053. [CrossRef]

221. Elbert, O.D.; Bullock, J.S.; Garrison-Kimmel, S.; Rocha, M.; Oñorbe, J.; Peter, A.H.G. Core formation in dwarf haloes with selfinteracting dark matter: No fine-tuning necessary. Mon. Not. R. Astron. Soc. 2015, 453, 29-37. doi: 10.1093/mnras/stv1470. [CrossRef]

222. Burkert, A. The Structure and Evolution of Weakly Self-interacting Cold Dark Matter Halos. Astrophys. J. Lett. 2000, 534, L143L146. doi: 10.1086/312674. [CrossRef]

223. Spergel, D.N.; Steinhardt, P.J. Observational Evidence for Self-Interacting Cold Dark Matter. Phys. Rev. Lett. 2000, 84, $3760-3763$. doi: 10.1103/PhysRevLett.84.3760. [CrossRef]

224. Hui, L.; Ostriker, J.P.; Tremaine, S.; Witten, E. Ultralight scalars as cosmological dark matter. Phys. Rev. D 2017, 95, 043541. doi: 10.1103/PhysRevD.95.043541. [CrossRef]

225. Hui, L.; Joyce, A.; Landry, M.J.; Li, X. Vortices and waves in light dark matter. J. Cosmol. Astropart. Phys. 2021, 2021, 011. doi: 10.1088/1475-7516/2021/01/011. [CrossRef]

226. Mocz, P.; Fialkov, A.; Vogelsberger, M.; Becerra, F.; Amin, M.A.; Bose, S.; Boylan-Kolchin, M.; Chavanis, P.H.; Hernquist, L.; Lancaster, L.; et al. First Star-Forming Structures in Fuzzy Cosmic Filaments. Phys. Rev. Lett. 2019, 123, 141301. doi: 10.1103/PhysRevLett.123.141301. [CrossRef]

227. Nori, M.; Murgia, R.; Iršič, V.; Baldi, M.; Viel, M. Lyman $\alpha$ forest and non-linear structure characterization in Fuzzy Dark Matter cosmologies. Mon. Not. R. Astron. Soc. 2019, 482, 3227-3243. doi: 10.1093/mnras/sty2888. [CrossRef]

228. Marsh, D.J.E.; Silk, J. A model for halo formation with axion mixed dark matter. Mon. Not. R. Astron. Soc. 2014, 437, 2652-2663. doi: 10.1093/mnras/stt2079. [CrossRef]

229. Chavanis, P.H. Derivation of a generalized Schrödinger equation for dark matter halos from the theory of scale relativity. Phys. Dark Univ. 2018, 22, 80-95. doi: 10.1016/j.dark.2018.09.004. [CrossRef]

230. Schive, H.Y.; Chiueh, T.; Broadhurst, T. Cosmic structure as the quantum interference of a coherent dark wave. Nat. Phys. 2014, 10, 496-499. doi: 10.1038/nphys2996. [CrossRef]

231. Boldrini, P.; Miki, Y.; Wagner, A.Y.; Mohayaee, R.; Silk, J.; Arbey, A. Cusp-to-core transition in low-mass dwarf galaxies induced by dynamical heating of cold dark matter by primordial black holes. Mon. Not. R. Astron. Soc. 2020, 492, 5218-5225. doi: 10.1093/mnras/staa150. [CrossRef]

232. Marsh, D.J.E. Axion cosmology. Phys. Rep. 2016, 643, 1-79. doi: 10.1016/j.physrep.2016.06.005. [CrossRef] 
233. Widrow, L.M.; Kaiser, N. Using the Schroedinger Equation to Simulate Collisionless Matter. Astrophys. J. Lett. 1993, 416, L71. doi: 10.1086/187073. [CrossRef]

234. Woo, T.P.; Chiueh, T. High-Resolution Simulation on Structure Formation with Extremely Light Bosonic Dark Matter. Astrophys. J. 2009, 697, 850-861. doi: 10.1088/0004-637X/697/1/850. [CrossRef]

235. Böhmer, C.G.; Harko, T. Can dark matter be a Bose Einstein condensate? J. Cosmol. Astropart. Phys. 2007, $2007,025$. doi: 10.1088/1475-7516/2007/06/025. [CrossRef]

236. Robles, V.H.; Bullock, J.S.; Boylan-Kolchin, M. Scalar field dark matter: Helping or hurting small-scale problems in cosmology? Mon. Not. R. Astron. Soc. 2019, 483, 289-298. doi: 10.1093/mnras/sty3190. [CrossRef]

237. Lee, J.W.; Lim, S. Minimum mass of galaxies from BEC or scalar field dark matter. J. Cosmol. Astropart. Phys. 2010, 2010, 007. doi: 10.1088/1475-7516/2010/01/007. [CrossRef]

238. El-Zant, A.A. Galaxy formation and dark matter: Small scale problems and quantum effects on astrophysical scales. J. Phys. Conf. Ser. 2019, 1253, 012007. doi: 10.1088/1742-6596/1253/1/012007. [CrossRef]

239. Veltmaat, J.; Schwabe, B.; Niemeyer, J.C. Baryon-driven growth of solitonic cores in fuzzy dark matter halos. Phys. Rev. D 2020, 101, 083518. doi: 10.1103/PhysRevD.101.083518. [CrossRef]

240. Safarzadeh, M.; Spergel, D.N. Ultra-light Dark Matter Is Incompatible with the Milky Way's Dwarf Satellites. Astrophys. J. 2020, 893, 21. doi: $10.3847 / 1538-4357 / a b 7 d b 2$. [CrossRef]

241. Davies, E.Y.; Mocz, P. Fuzzy dark matter soliton cores around supermassive black holes. Mon. Not. R. Astron. Soc. 2020, 492, 5721-5729. doi: 10.1093/mnras/staa202. [CrossRef]

242. Bar, N.; Blum, K.; Lacroix, T.; Panci, P. Looking for ultralight dark matter near supermassive black holes. J. Cosmol. Astropart. Phys. 2019, 2019, 045. doi: 10.1088/1475-7516/2019/07/045. [CrossRef]

243. Desjacques, V.; Nusser, A. Axion core-halo mass and the black hole-halo mass relation: constraints on a few parsec scales. Mon Not. R. Astron. Soc. 2019, 488, 4497-4503. doi: 10.1093/mnras/stz1978. [CrossRef]

244. Davoudiasl, H.; Denton, P.B. Ultralight Boson Dark Matter and Event Horizon Telescope Observations of M 87*. Phys. Rev. Lett. 2019, 123, 021102. doi: 10.1103/PhysRevLett.123.021102. [CrossRef]

245. Deng, H.; Hertzberg, M.P.; Namjoo, M.H.; Masoumi, A. Can light dark matter solve the core-cusp problem? Phys. Rev. D 2018, 98, 023513. doi: 10.1103/PhysRevD.98.023513. [CrossRef]

246. González-Morales, A.X.; Marsh, D.J.E.; Peñarrubia, J.; Ureña-López, L.A. Unbiased constraints on ultralight axion mass from dwarf spheroidal galaxies. Mon. Not. R. Astron. Soc. 2017, 472, 1346-1360. doi: 10.1093/mnras/stx1941. [CrossRef]

247. Marsh, D.J.E.; Pop, A.R. Axion dark matter, solitons and the cusp-core problem. Mon. Not. R. Astron. Soc. 2015, 451, $2479-2492$. doi: 10.1093/mnras/stv1050. [CrossRef]

248. Schive, H.Y.; Liao, M.H.; Woo, T.P.; Wong, S.K.; Chiueh, T.; Broadhurst, T.; Hwang, W.Y.P. Understanding the Core-Halo Relation of Quantum Wave Dark Matter from 3D Simulations. Phys. Rev. Lett. 2014, 113, 261302. doi: 10.1103/PhysRevLett.113.261302. [CrossRef]

249. De Martino, I.; Broadhurst, T.; Henry Tye, S.H.; Chiueh, T.; Schive, H.Y. Dynamical evidence of a dark solitonic core of $10^{9} \mathrm{M}_{\odot}$ in the milky way. Phys. Dark Univ. 2020, 28, 100503. doi: 10.1016/j.dark.2020.100503. [CrossRef]

250. Chen, S.R.; Schive, H.Y.; Chiueh, T. Jeans analysis for dwarf spheroidal galaxies in wave dark matter. Mon. Not. R. Astron. Soc. 2017, 468, 1338-1348. doi: 10.1093/mnras/stx449. [CrossRef]

251. Martin, N.F.; Ibata, R.A.; Collins, M.L.M.; Rich, R.M.; Bell, E.F.; Ferguson, A.M.N.; Laevens, B.P.M.; Rix, H.W.; Chapman, S.C.; Koch, A. Triangulum II: A Very Metal-poor and Dynamically Hot Stellar System. Astrophys. J. 2016, 818, 40. doi: 10.3847/0004-637X/818/1/40. [CrossRef]

252. Di Paolo, C.; Salucci, P. Fundamental properties of the dark and the luminous matter from Low Surface Brightness discs. arXiv 2020, arXiv:2005.03520.

253. Salucci, P.; Burkert, A. Dark Matter Scaling Relations. Astrophys. J. Lett. 2000, 537, L9-L12. doi: 10.1086/312747. [CrossRef]

254. Burkert, A. Fuzzy Dark Matter and Dark Matter Halo Cores. Astrophys. J. 2020, 904, 161. doi: 10.3847/1538-4357/abb242. [CrossRef]

255. Mina, M.; Mota, D.F.; Winther, H.A. Solitons in the dark: Non-linear structure formation with fuzzy dark matter. arXiv 2020, arXiv:2007.04119.

256. Jowett Chan, H.Y.; Ferreira, E.G.M.; May, S.; Hayashi, K.; Chiba, M. The Diversity of Core Halo Structure in the Fuzzy Dark Matter Model. arXiv 2021, arXiv:2110.11882.

257. Mocz, P.; Vogelsberger, M.; Robles, V.H.; Zavala, J.; Boylan-Kolchin, M.; Fialkov, A.; Hernquist, L. Galaxy formation with BECDMI. Turbulence and relaxation of idealized haloes. Mon. Not. R. Astron. Soc. 2017, 471, 4559-4570. doi: 10.1093/mnras/stx1887. [CrossRef]

258. Schwabe, B.; Niemeyer, J.C.; Engels, J.F. Simulations of solitonic core mergers in ultralight axion dark matter cosmologies. Phys. Rev. D 2016, 94, 043513. doi: 10.1103/PhysRevD.94.043513. [CrossRef]

259. Nori, M.; Baldi, M. Scaling relations of fuzzy dark matter haloes-I. Individual systems in their cosmological environment. Mon. Not. R. Astron. Soc. 2021, 501, 1539-1556. doi: 10.1093/mnras/staa3772. [CrossRef]

260. de Laix, A.A.; Scherrer, R.J.; Schaefer, R.K. Constraints on Self-interacting Dark Matter. Astrophys. J. 1995, $452,495$. doi: 10.1086/176322. [CrossRef]

261. Carlson, E.D.; Machacek, M.E.; Hall, L.J. Self-interacting Dark Matter. Astrophys. J. 1992, 398, 43. doi: 10.1086/171833. [CrossRef]

262. Robertson, A.; Massey, R.; Eke, V. What does the Bullet Cluster tell us about self-interacting dark matter? Mon. Not. R. Astron. Soc. 2017, 465, 569-587. doi: 10.1093/mnras/stw2670. [CrossRef] 
263. Rocha, M.; Peter, A.H.G.; Bullock, J.S.; Kaplinghat, M.; Garrison-Kimmel, S.; Oñorbe, J.; Moustakas, L.A. Cosmological simulations with self-interacting dark matter-I. Constant-density cores and substructure. Mon. Not. R. Astron. Soc. 2013, 430, 81-104. doi: 10.1093/mnras/sts514. [CrossRef]

264. Peter, A.H.G.; Rocha, M.; Bullock, J.S.; Kaplinghat, M. Cosmological simulations with self-interacting dark matter-II. Halo shapes versus observations. Mon. Not. R. Astron. Soc. 2013, 430, 105-120. doi: 10.1093/mnras/sts535. [CrossRef]

265. Vogelsberger, M.; Zavala, J.; Loeb, A. Subhaloes in self-interacting galactic dark matter haloes. Mon. Not. R. Astron. Soc. 2012, 423, 3740-3752. doi: 10.1111/j.1365-2966.2012.21182.x. [CrossRef]

266. Colín, P.; Avila-Reese, V.; Valenzuela, O.; Firmani, C. Structure and Subhalo Population of Halos in a Self-interacting Dark Matter Cosmology. Astrophys. J. 2002, 581, 777-793. doi: 10.1086/344259. [CrossRef]

267. Davé, R.; Spergel, D.N.; Steinhardt, P.J.; Wandelt, B.D. Halo Properties in Cosmological Simulations of Self-interacting Cold Dark Matter. Astrophys. J. 2001, 547, 574-589. doi: 10.1086/318417. [CrossRef]

268. Yoshida, N.; Springel, V.; White, S.D.M.; Tormen, G. Weakly Self-interacting Dark Matter and the Structure of Dark Halos. Astrophys. J. Lett. 2000, 544, L87-L90. doi: 10.1086/317306. [CrossRef]

269. Yoshida, N.; Springel, V.; White, S.D.M.; Tormen, G. Collisional Dark Matter and the Structure of Dark Halos. Astrophys. J. Lett. 2000, 535, L103-L106. doi: 10.1086/312707. [CrossRef]

270. D'Onghia, E.; Burkert, A. The Failure of Self-Interacting Dark Matter to Solve the Overabundance of Dark Satellites and the Soft Core Question. Astrophys. J. 2003, 586, 12-16. doi: 10.1086/367606. [CrossRef]

271. Tulin, S.; Yu, H.B. Dark matter self-interactions and small scale structure. Phys. Rep. 2018, 730, 1-57. j.physrep.2017.11.004. [CrossRef]

272. Kaplinghat, M.; Tulin, S.; Yu, H.B. Dark Matter Halos as Particle Colliders: Unified Solution to Small-Scale Structure Puzzles from Dwarfs to Clusters. Phys. Rev. Lett. 2016, 116, 041302. doi: 10.1103/PhysRevLett.116.041302. [CrossRef]

273. Zavala, J.; Lovell, M.R.; Vogelsberger, M.; Burger, J.D. Diverse dark matter density at sub-kiloparsec scales in Milky Way satellites: Implications for the nature of dark matter. Phys. Rev. D 2019, 100, 063007. doi: 10.1103/PhysRevD.100.063007. [CrossRef]

274. Loeb, A.; Weiner, N. Cores in Dwarf Galaxies from Dark Matter with a Yukawa Potential. Phys. Rev. Lett. 2011, 106, 171302. doi: 10.1103/PhysRevLett.106.171302. [CrossRef]

275. Feng, J.L.; Kaplinghat, M.; Yu, H.B. Halo-Shape and Relic-Density Exclusions of Sommerfeld-Enhanced Dark Matter Explanations of Cosmic Ray Excesses. Phys. Rev. Lett. 2010, 104, 151301. doi: 10.1103/PhysRevLett.104.151301. [CrossRef] [PubMed]

276. Lynden-Bell, D.; Wood, R. The gravo-thermal catastrophe in isothermal spheres and the onset of red-giant structure for stellar systems. Mon. Not. R. Astron. Soc. 1968, 138, 495. doi: 10.1093/mnras/138.4.495. [CrossRef]

277. Balberg, S.; Shapiro, S.L.; Inagaki, S. Self-Interacting Dark Matter Halos and the Gravothermal Catastrophe. Astrophys. J. 2002, 568, 475-487. doi: 10.1086/339038. [CrossRef]

278. Koda, J.; Shapiro, P.R. Gravothermal collapse of isolated self-interacting dark matter haloes: N-body simulation versus the fluid model. Mon. Not. R. Astron. Soc. 2011, 415, 1125-1137. doi: 10.1111/j.1365-2966.2011.18684.x. [CrossRef]

279. Pollack, J.; Spergel, D.N.; Steinhardt, P.J. Supermassive Black Holes from Ultra-strongly Self-interacting Dark Matter. Astrophys. J. 2015, 804, 131. doi: 10.1088/0004-637X/804/2/131. [CrossRef]

280. Nishikawa, H.; Boddy, K.K.; Kaplinghat, M. Accelerated core collapse in tidally stripped self-interacting dark matter halos. Phys. Rev. D 2020, 101, 063009. doi: 10.1103/PhysRevD.101.063009. [CrossRef]

281. Sameie, O.; Yu, H.B.; Sales, L.V.; Vogelsberger, M.; Zavala, J. Self-Interacting Dark Matter Subhalos in the Milky Way's Tides. Phys. Rev. Lett. 2020, 124, 141102. doi: 10.1103/PhysRevLett.124.141102. [CrossRef]

282. Kahlhoefer, F.; Kaplinghat, M.; Slatyer, T.R.; Wu, C.L. Diversity in density profiles of self-interacting dark matter satellite halos. J. Cosmol. Astropart. Phys. 2019, 2019, 010. doi: 10.1088/1475-7516/2019/12/010. [CrossRef]

283. Fry, A.B.; Governato, F.; Pontzen, A.; Quinn, T.; Tremmel, M.; Anderson, L.; Menon, H.; Brooks, A.M.; Wadsley, J. All about baryons: Revisiting SIDM predictions at small halo masses. Mon. Not. R. Astron. Soc. 2015, 452, 1468-1479. doi: 10.1093/mnras/stv1330. [CrossRef]

284. Elbert, O.D.; Bullock, J.S.; Kaplinghat, M.; Garrison-Kimmel, S.; Graus, A.S.; Rocha, M. A Testable Conspiracy: Simulating Baryonic Effects on Self-interacting Dark Matter Halos. Astrophys. J. 2018, 853, 109. doi: 10.3847/1538-4357/aa9710. [CrossRef]

285. Kaplinghat, M.; Keeley, R.E.; Linden, T.; Yu, H.B. Tying Dark Matter to Baryons with Self-Interactions. Phys. Rev. Lett. 2014, 113, 021302. doi: 10.1103/PhysRevLett.113.021302. [CrossRef]

286. Kamada, A.; Kaplinghat, M.; Pace, A.B.; Yu, H.B. Self-Interacting Dark Matter Can Explain Diverse Galactic Rotation Curves. Phys. Rev. Lett. 2017, 119, 111102. doi: 10.1103/PhysRevLett.119.111102. [CrossRef]

287. Creasey, P.; Sameie, O.; Sales, L.V.; Yu, H.B.; Vogelsberger, M.; Zavala, J. Spreading out and staying sharp-Creating diverse rotation curves via baryonic and self-interaction effects. Mon. Not. R. Astron. Soc. 2017, 468, 2283-2295. doi: 10.1093/mnras/stx522. [CrossRef]

288. Ren, T.; Kwa, A.; Kaplinghat, M.; Yu, H.B. Reconciling the Diversity and Uniformity of Galactic Rotation Curves with SelfInteracting Dark Matter. Phys. Rev. X 2019, 9, 031020. doi: 10.1103/PhysRevX.9.031020. [CrossRef]

289. Kaplinghat, M.; Ren, T.; Yu, H.B. Dark Matter Cores and Cusps in Spiral Galaxies and their Explanations. arXiv 2019, arXiv:1911.00544.

290. Fitts, A.; Boylan-Kolchin, M.; Bozek, B.; Bullock, J.S.; Graus, A.; Robles, V.; Hopkins, P.F.; El-Badry, K.; Garrison-Kimmel, S.; Faucher-Giguère, C.A.; et al. Dwarf galaxies in CDM, WDM, and SIDM: disentangling baryons and dark matter physics. Mon. Not. R. Astron. Soc. 2019, 490, 962-977. doi: 10.1093/mnras/stz2613. [CrossRef] 
291. Lelli, F.; McGaugh, S.S.; Schombert, J.M. SPARC: Mass Models for 175 Disk Galaxies with Spitzer Photometry and Accurate Rotation Curves. Astron. J. 2016, 152, 157. doi: 10.3847/0004-6256/152/6/157. [CrossRef]

292. Zel'dovich, Y.B.; Novikov, I.D. The Hypothesis of Cores Retarded during Expansion and the Hot Cosmological Model. Sov. Astron. 1967, 10, 602

293. Hawking, S. Gravitationally collapsed objects of very low mass. Mon. Not. R. Astron. Soc. $1971,152,75$. mnras/152.1.75. [CrossRef]

294. Khlopov, M.Y. Primordial black holes. Res. Astron. Astrophys. 2010, 10, 495-528. doi: 10.1088/1674-4527/10/6/001. [CrossRef]

295. Clesse, S.; García-Bellido, J. Seven hints for primordial black hole dark matter. Phys. Dark Univ. 2018, 22, 137-146. doi: 10.1016/j.dark.2018.08.004. [CrossRef]

296. Inomata, K.; Kawasaki, M.; Mukaida, K.; Tada, Y.; Yanagida, T.T. Inflationary primordial black holes as all dark matter. Phys. Rev. D 2017, 96, 043504. doi: 10.1103/PhysRevD.96.043504. [CrossRef]

297. Clesse, S.; García-Bellido, J. Massive primordial black holes from hybrid inflation as dark matter and the seeds of galaxies. Phys. Rev. D 2015, 92, 023524. doi: 10.1103/PhysRevD.92.023524. [CrossRef]

298. Carr, B.; Kuhnel, F. Primordial Black Holes as Dark Matter Candidates. arXiv 2021, arXiv:2110.02821.

299. Carr, B.; Raidal, M.; Tenkanen, T.; Vaskonen, V.; Veermäe, H. Primordial black hole constraints for extended mass functions. Phys. Rev. D 2017, 96, 023514. doi: 10.1103/PhysRevD.96.023514. [CrossRef]

300. Abbott, B.P.; Abbott, R.; Abbott, T.D.; Abernathy, M.R.; Acernese, F.; Ackley, K.; Adams, C.; Adams, T.; Addesso, P.; Adhikari, R.X.; et al. Observation of Gravitational Waves from a Binary Black Hole Merger. Phys. Rev. Lett. 2016, 116, 061102. doi: 10.1103/PhysRevLett.116.061102. [CrossRef]

301. Amaro-Seoane, P.; Audley, H.; Babak, S.; Baker, J.; Barausse, E.; Bender, P.; Berti, E.; Binetruy, P.; Born, M.; Bortoluzzi, D.; et al Laser Interferometer Space Antenna. arXiv 2017, arXiv:1702.00786.

302. Spitzer, L., Jr. Equipartition and the Formation of Compact Nuclei in Spherical Stellar Systems. Astrophys. J. Lett. 1969, 158, L139. doi: 10.1086/180451. [CrossRef]

303. Chandrasekhar, S. Stochastic Problems in Physics and Astronomy. Rev. Mod. Phys. 1943, 15, 1-89. RevModPhys.15.1. [CrossRef]

304. Brandt, T.D. Constraints on MACHO Dark Matter from Compact Stellar Systems in Ultra-faint Dwarf Galaxies. Astrophys. J. Lett. 2016, 824, L31. doi: 10.3847/2041-8205/824/2/L31. [CrossRef]

305. Koushiappas, S.M.; Loeb, A. Dynamics of Dwarf Galaxies Disfavor Stellar-Mass Black Holes as Dark Matter. Phys. Rev. Lett. 2017, 119, 041102. doi: 10.1103/PhysRevLett.119.041102. [CrossRef]

306. Zhu, Q.; Vasiliev, E.; Li, Y.; Jing, Y. Primordial black holes as dark matter: constraints from compact ultra-faint dwarfs. Mon. Not. R. Astron. Soc. 2018, 476, 2-11. doi: 10.1093/mnras/sty079. [CrossRef]

307. Inman, D.; Ali-Haïmoud, Y. Early structure formation in primordial black hole cosmologies. Phys. Rev. D 2019, 100, 083528. doi: 10.1103/PhysRevD.100.083528. [CrossRef]

308. Collaboration, G.; Brown, A.G.A.; Vallenari, A.; Prusti, T.; de Bruijne, J.H.J.; Babusiaux, C.; Biermann, M.; Creevey, O.L.; Evans, D.W.; Eyer, L.; et al. Gaia Early Data Release 3. Summary of the contents and survey properties. Astron. Astrophys. 2021, 649, A1. doi: 10.1051/0004-6361/202039657. [CrossRef]

309. Ebisu, T.; Ishiyama, T.; Hayashi, K. Constraining Self-Interacting Dark Matter with Dwarf Spheroidal Galaxies and High-resolution Cosmological N-body Simulations. arXiv 2021, arXiv:2107.05967.

310. Burger, J.D.; Zavala, J.; Sales, L.V.; Vogelsberger, M.; Marinacci, F.; Torrey, P. Degeneracies Between Self-interacting Dark Matter and Supernova Feedback as cusp-core transformation mechanisms. arXiv 2021, arXiv:2108.07358. 